TRANSACTIONS OF THE

AMERICAN MATHEMATICAL SOCIETY

Volume 315, Number 1, September 1989

\title{
HIGHER-ORDER SUGAWARA OPERATORS FOR AFFINE LIE ALGEBRAS
}

\author{
ROE GOODMAN AND NOLAN R. WALLACH
}

\begin{abstract}
Let $\hat{\mathfrak{g}}$ be the affine Lie algebra associated to a simple Lie algebra $\mathfrak{g}$. Representations of $\hat{\mathfrak{g}}$ are described by current fields $X(\zeta)$ on the circle $\mathbf{T}(X \in \mathfrak{g}$ and $\zeta \in \mathbf{T})$. In this paper a linear map $\sigma$ from the symmetric algebra $S(\mathfrak{g})$ to (formal) operator fields on a suitable category of $\hat{\mathfrak{g}}$ modules is constructed. The operator fields corresponding to $\mathfrak{g}$-invariant elements of $S(\mathfrak{g})$ are called Sugawara fields. It is proved that they satisfy commutation relations of the form
\end{abstract}

$(*) \quad[\sigma(u)(\zeta), X(\eta)]=c_{\infty} D \delta(\zeta / \eta) \sigma\left(\nabla_{X} u\right)(\zeta)+$ higher-order terms

with the current fields, where $c_{\infty}$ is a renormalization of the central element in $\hat{\mathfrak{g}}$ and $D \delta$ is the derivative of the Dirac delta function. The higher-order terms in $(*)$ are studied using results from invariant theory and finite-dimensional representation theory of $\mathfrak{g}$. For suitably normalized invariants $u$ of degree 4 or less, these terms are shown to be zero. This vanishing is also proved for $\mathfrak{g}=\operatorname{sl}(n, \mathrm{C})$ and $u$ running over a particular choice of generators for the symmetric invariants. The Sugawara fields defined by such invariants commute with the current fields whenever $c_{\infty}$ is represented by zero. This property is used to obtain the commuting ring, composition series, and character formulas for a class of highest-weight modules for $\hat{\mathfrak{g}}$.

\section{INTRODUCTION}

Let $\hat{\mathfrak{g}}$ be the affine algebra associated with a finite-dimensional simple Lie algebra $\mathfrak{g}$. The representation theory of $\hat{\mathfrak{g}}$ is closely related to models for quantum field theory in two space-time dimensions. In the so-called Sugawara models, the representation of $\hat{\mathfrak{g}}$ corresponds to a current field, and the energymomentum field is obtained as a quadratic function of the current fields [Fre, G-O, P-S, Sug]. In this paper we construct a linear map from the symmetric algebra $S(\mathfrak{g})$ to (formal) operator fields on a suitable category of $\hat{\mathfrak{g}}$ modules. We call the operator fields corresponding to $\mathfrak{g}$-invariant elements of $S(\mathfrak{g})$ Sugawara fields. Our main results concern the commutation relations between current fields and Sugawara fields.

Received by the editors March 2, 1988. Presented to the 848th meeting of the AMS, Worcester, Massachusetts, April 16, 1989.

1980 Mathematics Subject Classification (1985 Revision). Primary 17B35, 17B67, 22E47; Secondary 15A72, 20C30, 20G45, 81E99.

Research partially supported by Rutgers University Faculty Academic Study Program, Australian National University Centre for Mathematical Analysis, and NSF Grant DMS 86-03169 (to R.G.) and by NSF Grant DMS 84-02684 (to N.R.W.). 
To describe our results in more detail, we recall that $\hat{\mathfrak{g}}$ is a central extension of the Lie algebra $\tilde{\mathfrak{g}}=\mathbf{C}\left[\zeta, \zeta^{-1}\right] \otimes \mathfrak{g}$. Representations of $\hat{\mathfrak{g}}$ arise from projective representations of the current algebra $\tilde{\mathfrak{g}}$, which we view as the maps from the circle $\mathbf{T}$ to $\mathfrak{g}$ with finite Fourier series (taking $\zeta=e^{i \theta}$ on $\mathbf{T}$ ). If $\pi$ is such a representation, then $\pi(\phi \otimes X)$, for $\phi \in \mathbf{C}\left[\zeta, \zeta^{-1}\right]$ and $X \in \mathfrak{g}$, can be (formally) expressed in terms of a current field $X(\zeta)$ on $\mathbf{T}$ as

$$
\pi(\phi \otimes X)=\int_{\mathbf{T}} \phi\left(e^{i \theta}\right) X\left(e^{i \theta}\right) d \theta .
$$

The operator-valued distribution $X(\zeta)$ is (formally) defined as $\pi(\delta \otimes X)$, where

$$
\delta(\zeta)=\sum_{n \in \mathbf{Z}} \zeta^{n}
$$

is the Dirac function on $\mathbf{T}$. The current fields satisfy the commutation relations

$$
[X(\zeta), Y(\eta)]=\delta(\zeta / \eta)[X, Y](\zeta)+c D \delta(\zeta / \eta) B(X, Y)
$$

where $B$ is the Killing form on $\mathfrak{g}, c$ is a basis for the center of $\hat{\mathfrak{g}}$, and $D$ is the vector field $\zeta \partial / \partial \zeta$ on $\mathbf{T}$.

The formal simplicity of the commutation relations (2) makes the current fields a powerful tool for studying representations of affine algebras (cf. [L-W]). This apparent simplicity has its price, however. The passage from the representation $\pi$ to the current field $X(\zeta)$ and the commutation relations of the current fields both involve the singular function $\delta$ and its derivatives. More generally, formal products of current fields evaluated at a point contain divergent expressions which must be "renormalized" in some consistent way to become meaningful (cf. [C-G-J]). In this paper we restrict attention to a category $\mathscr{F}$ of $\hat{\mathfrak{g}}$ modules $M$ on which the current fields and the renormalization can be defined purely algebraically. We define products of the fields by symmetrization and normal ordering relative to the positive-negative splitting of formal Fourier series. Given an element $u$ in the symmetric algebra $S(\mathfrak{g})$ we obtain by this procedure a well-defined formal operator field that we denote by $\sigma(u)(\zeta)$. (For $u=X^{n}$ a pure power, this field is conventionally denoted by $: X(\zeta)^{n}:$ in the physics literature.) For $\phi \in \mathbf{C}\left[\zeta, \zeta^{-1}\right]$, the smeared field

$$
J_{u}(\phi)=\int_{\mathbf{T}} \phi\left(e^{i \theta}\right) \sigma(u)\left(e^{i \theta}\right) d \theta
$$

is a well-defined operator on $M$. In particular, for $u=X \in \mathfrak{g}$, we have $\sigma(X)(\zeta)=X(\zeta)$ and the operator $J_{X}(\phi)$ gives the action of $\phi \otimes X$ on $M$.

To describe the commutation relations between these fields and the currents, we recall from classical invariant theory that if $u \in S(\mathfrak{g})$ then the polarization of $u$ is the map $X \rightarrow \nabla_{X} u$ from $\mathfrak{g}$ to $S(\mathfrak{g})$, where $\nabla$ denotes the gradient operator [Wey]. The first main result of the paper is the calculation of the commutator between the Sugawara field defined by $u \in S(\mathfrak{g})^{G}$ and the current defined by $X \in \mathfrak{g}$. We prove (Corollary 3.12) that the commutation relations can be expressed as

$$
[\sigma(u)(\zeta), X(\eta)]=c_{\infty} D \delta(\zeta / \eta) \sigma\left(\nabla_{X} u\right)(\zeta)+\text { higher-order terms }
$$


where $c_{\infty}$ is the renormalized value $c+\rho(c)$. Here $\rho$ is the functional taking value 1 on the canonical generators of a Cartan subalgebra of $\hat{\mathfrak{g}}$. The renormalization shift $\rho(c)$ is determined by the value of the quadratic Casimir operator for $\mathfrak{g}$ in the adjoint representation and hence reflects the noncommutativity of $\mathfrak{g}$. (With the element $c$ defined as in (1) via the Killing form $B, \rho(c)=\frac{1}{2}$; with another natural normalization of $c, \rho(c)$ is the dual Coxeter number of g (cf. [G-O]).)

We call $\sigma(u)(\zeta)$ a restricted Sugawara field when the higher-order terms vanish in equation (4). It is well known that this happens for the quadratic invariant $Q=\sum X_{i}^{2}$, where $\left\{X_{i}\right\}$ is a $B$-orthonormal basis for $\mathfrak{g}$. In this paper we determine sufficient conditions on higher-order invariants $u$ for $\sigma(u)(\zeta)$ to be a restricted Sugawara field (Theorem 4.1, Corollary 4.3, and Theorem 4.4). Observe that for a restricted Sugawara field, the integrated form of (4) gives the operator commutation relations

$$
\left[J_{u}(\phi), J_{X}(\psi)\right]=c_{\infty} J_{\nabla_{X} u}(\phi D \psi)
$$

where $\phi, \psi \in \mathbf{C}\left[\zeta, \zeta^{-1}\right]$. In particular, when $u=Q$ as above, then $\nabla_{X} Q=2 X$ and equation (5) becomes

$$
\left[J_{Q}(\phi), J_{X}(\psi)\right]=2 c_{\infty} J_{X}(\phi D \psi) .
$$

Thus the following dichotomy arises: If $c_{\infty}$ is invertible on the module $M$ then the operator $1 /\left(2 c_{\infty}\right) J_{Q}(\phi)$ on $M$ implements the action of the vector field $\phi D$ on $\hat{\mathfrak{g}}$ (cf. [G-W3]). However, if $c_{\infty}=0$ on $M$ then every restricted Sugawara field commutes with the action of $\hat{\mathfrak{g}}$ on $M$. Hence on the category of $\hat{\mathfrak{g}}$ modules where $c_{\infty}$ acts by 0 , the restricted Sugawara fields can provide a substitute for the center of the (completed) enveloping algebra of $\hat{\mathfrak{g}}$. In the case of highest-weight modules in the category $\mathscr{O}$ (cf. $[\mathrm{K}-\mathrm{K}]$ ), the condition $c_{\infty}=0$ corresponds to highest weights on the boundary of the translated Tits cone, where the operators constructed in [Kac] do not exist (cf. [C-I] for general negative results concerning the center of the enveloping algebra of $\hat{\mathfrak{g}}$ ).

The paper is organized as follows: In $\S 1$ we obtain trace identities for the iterated polarizations of invariant polynomials on $\mathfrak{g}$. In $\S \S 2$ and 3 we develop the formal algebraic theory of Sugawara fields and obtain the general commutator formula (4), with a general description of the form of the higher-order terms. In $\S 4$ we use results from classical invariant theory and finite-dimensional representation theory to obtain a complete family of restricted Sugawara operators when $\mathfrak{g}$ is of type $A_{l}$ or classical of rank $\leq 2$. We then use these operators (following the broad lines of [Wal]) to analyze the characters of the irreducible objects in the subcategory $\mathscr{O}_{I}$ of $\mathscr{O}$ where $c_{\infty}=0$.

Although our results concerning the existence of restricted Sugawara operators are complete only in the case of type $A_{l}$ or for invariants $u$ of degree $\leq 4$, our methods are completely general. Of possible independent interest is the material in $\S 3$ of the paper on "nonabelian vertex operators," which extends the formal analysis of [L-W] to the nonabelian case. There is also a substantial 
study of invariant theory in the paper. Most of the results in invariant theory that we prove for type $A_{l}$ we have also extended to the other classical groups, and we expect this to be useful in other contexts.

Our method of constructing a complete set of restricted Sugawara operators for $A_{l}$ and $B_{2}$ uses explicit commutator formulas up to degree 3 in ad $X$, for $X \in \mathfrak{g}$. The higher-order terms in the commutator (4) are shown to vanish using the results from invariant theory. For general algebras of type $B_{l}, C_{l}$, and $D_{l}$ one must in fact have more information on the term of degree 4 in ad $X$ in order to apply our invariant-theoretic methods and results.

Affine Lie algebras and groups are also closely related to certain completely integrable finite-particle systems (cf. [G-W2, G-W4]), and this connection was our original motivation for constructing higher-order Sugawara operators. In future work we hope to apply the higher-order Sugawara operators constructed in this paper to the quantized periodic Toda systems studied in [G-W4], using representation-theoretic methods as was done for the nonperiodic systems in [Kos] and [G-W1].

After finishing this paper, we received the preprint Sugawara operators and Kac-Kazhdan conjecture by $\mathrm{T}$. Hayashi. In this preprint results similar to our Theorems 4.1 and 5.2 (with weaker hypotheses on the highest weight) are stated for $\mathfrak{g}$ of type $A_{l}, B_{l}$, or $C_{l}$ (complete proofs are given only for the case of $A_{l}$ ). The Sugawara operators corresponding to basic invariants of degree 3 or 4 that Hayashi constructs are the homogeneous components of the restricted Sugawara fields that we obtain in Theorem 4.1 and Corollary 4.3. However, it is unclear what the relation is between Hayashi's "Sugawara operators" of order greater than 4 and the higher-order restricted Sugawara fields that we construct in Theorem 4.11 for $\mathfrak{g}$ of type $A_{l}$.

\section{TRACE IDENTITIES FOR INVARIANT POLYNOMIALS}

1.1. Polarization. Let $\mathfrak{g}$ be a finite-dimensional reductive Lie algebra over $\mathbf{C}$. Fix an invariant nondegenerate bilinear form $B$ on $\mathfrak{g}$. Let $S(\mathfrak{g})$ be the symmetric algebra over $\mathfrak{g}$, and let $S^{k}(\mathfrak{g})$ be the subspace of elements homogeneous of degree $k$. For $X \in \mathfrak{g}$ let $\nabla_{X}$ be the derivation of $S(\mathfrak{g})$ such that

$$
\nabla_{X}(Y)=B(X, Y), \quad \text { for } Y \in \mathfrak{g} \text {. }
$$

Let $\operatorname{ad}(X)$ be the derivation of $S(\mathfrak{g})$ such that

$$
\operatorname{ad}(X)(Y)=[X, Y], \quad \text { for } Y \in \mathfrak{g} .
$$

We denote by $S(\mathfrak{g})^{\mathfrak{g}}$ the subalgebra of $S(\mathfrak{g})$ annihilated by $\operatorname{ad}(\mathfrak{g})$.

Lemma 1.1. For all $X, Y \in \mathfrak{g}$, one has $\left[\operatorname{ad} X, \nabla_{Y}\right]=\nabla_{[X, Y]}$.

Proof. It is enough to check this equation on an element $Z \in \mathfrak{g}$, since both sides of the equation are derivations. But $\nabla_{Y} Z$ is a constant, so it is annihilated by ad $X$. On the other hand,

$$
\nabla_{Y}(\operatorname{ad} X(Z))=B(Y,[X, Z])=-B([X, Y], Z)=-\nabla_{[X, Y]} Z,
$$

by the invariance of the form $B$. 
Corollary 1.2. If $u \in S(\mathfrak{g})^{\mathfrak{g}}$ then $(\operatorname{ad} X)\left(\nabla_{Y} u\right)=\nabla_{\mathrm{ad} X(Y)} u$. Thus the map $T_{u}: Y \rightarrow \nabla_{Y} u$ is in $\operatorname{Hom}_{\mathfrak{g}}(\mathfrak{g}, S(\mathfrak{g}))$.

Remark. In [Wey], the map $T_{u}$ is called the polarization of the invariant $u$.

Fix an orthonormal basis $\left\{X_{i}\right\}$ for $\mathfrak{g}$ relative to the form $B$ and write $\nabla_{i}=\nabla_{X_{i}}$.

Lemma 1.3. If $u \in S(\mathfrak{g})^{\mathfrak{g}}$, then for all $Y \in \mathfrak{g}$,

$$
\sum_{i}\left(\nabla_{i} u\right) \operatorname{ad} X_{i}(Y)=0 .
$$

Proof. We may assume that $u$ is homogeneous of degree $j$. Denote by $(\cdot, \cdot)$ the invariant bilinear form on $S^{j}(\mathfrak{g})$ such that

$$
\left(X^{j}, Y^{j}\right)=B(X, Y)^{j}
$$

for $X, Y \in \mathfrak{g}$. This form is nondegenerate, and for $Z \in \mathfrak{g}$ we have

$$
\begin{aligned}
\sum_{i}\left(\left(\nabla_{i} u\right) \operatorname{ad} X_{i}(Y), Z^{j}\right) & =\sum_{i}\left(\nabla_{i} u, Z^{j-1}\right)\left([Y, Z], X_{i}\right) \\
& =\left(\nabla_{[Y, Z]} u, Z^{j-1}\right),
\end{aligned}
$$

since $\left\{X_{i}\right\}$ is an orthonormal basis and the map $X \rightarrow \nabla_{X}$ is C-linear. But by Corollary 1.2, $\nabla_{[Y, Z]} u=-\operatorname{ad} Z\left(\nabla_{Y} u\right)$. Hence the left side of $(1)$ is

$$
-\left(\operatorname{ad} Z\left(\nabla_{Y} u\right), Z^{j-1}\right)=\left(\nabla_{Y} u, \operatorname{ad} Z\left(Z^{j-1}\right)\right)=0 .
$$

Since the elements $Z^{j}$ span $S^{j}(\mathfrak{g})$ as $Z$ runs over $\mathfrak{g}$, this last equation proves the lemma.

Let $G$ be a compact connected Lie group whose complexified Lie algebra is $\mathfrak{g}$. Then $G$ acts on $\mathfrak{g}$ and on $S(\mathfrak{g})$ by the complexification of the adjoint representation, and we shall write

$$
X^{g}=\operatorname{Ad}\left(g^{-1}\right) X, \quad \text { for } X \in \mathfrak{g} \text { and } g \in G .
$$

Since $G$ is connected, we have $S(\mathfrak{g})^{\mathfrak{g}}=S(\mathfrak{g})^{G}$; i.e., an element $u \in S(\mathfrak{g})$ is annihilated by $\mathfrak{g}$ if and only if it is fixed by $G$.

Denote the normalized invariant measure on $G$ by $d g$. Then for any $X \in \mathfrak{g}$ and any positive integer $n$, the homogeneous element

$$
I\left(X^{n}\right)=\int_{G}\left(X^{g}\right)^{n} d g
$$

is in $S(\mathfrak{g})^{G}$. Fix $n \geq 0$. Write $u=I\left(X^{n+1}\right)$. Then

$$
\nabla_{i} u=(n+1) \int_{G}\left(X^{g}\right)^{n} B\left(X^{g}, X_{i}\right) d g .
$$

Using (3) in Lemma 1.3 and the expansion

$$
\operatorname{ad} X^{g}=\sum_{i} B\left(X^{g}, X_{i}\right) \operatorname{ad} X_{i},
$$

we obtain the following identity: 
Corollary 1.4. For all $X, Y \in \mathfrak{g}$ and all integers $n \geq 0$,

$$
\int_{G}\left(X^{g}\right)^{n}\left(\operatorname{ad} X^{g}\right)(Y) d g=0 .
$$

1.2. Trace identities. We now obtain a basic "trace identity" for the polarization process.

Proposition 1.5. Let $u \in S(\mathfrak{g})^{G}$. Then for any integer $p \geq 2$ and $Y \in \mathfrak{g}$,

$$
\begin{aligned}
& \sum_{i_{1}, \ldots, i_{p}}\left(\nabla_{i_{1}} \cdots \nabla_{i_{p}} u\right) \operatorname{ad} X_{i_{1}} \cdots \operatorname{ad} X_{i_{p}}(Y) \\
& \quad=\sum_{i_{1}, \ldots, i_{p-1}}\left(\nabla_{i_{1}} \cdots \nabla_{i_{p-1}} u\right) \operatorname{tr}\left(\operatorname{ad} X_{i_{1}} \cdots \operatorname{ad} X_{i_{p-1}} \operatorname{ad} Y\right) .
\end{aligned}
$$

Proof. We shall prove $(1)_{p}$ by induction on $p$. Denote the left side of $(1)_{p}$ by $S_{p}$. Observe that because $\nabla_{i}$ is a derivation and $\nabla_{i} \nabla_{j}=\nabla_{j} \nabla_{i}$, we have

$$
\left(\nabla_{i} \nabla_{j} u\right) Z=\nabla_{j}\left\{\left(\nabla_{i} u\right) Z\right\}-\left(\nabla_{i} u\right)\left(\nabla_{j} Z\right) \text {. }
$$

Thus when $p=2$ we may write

$$
S_{2}=\sum_{j} \nabla_{j}\left\{\sum_{i}\left(\nabla_{i} u\right) \operatorname{ad} X_{i}\left(\operatorname{ad} X_{j}(Y)\right)\right\}-\sum_{i, j}\left(\nabla_{i} u\right)\left(\nabla_{j}\left(\operatorname{ad} X_{i} \operatorname{ad} X_{j}(Y)\right)\right. \text {. }
$$

Now the first group of terms vanishes by Lemma 1.3. Since $\nabla_{j} Z=B\left(X_{j}, Z\right)$ for $Z \in \mathfrak{g}$, we thus have

$$
\begin{aligned}
S_{2} & =-\sum_{i, j}\left(\nabla_{i} u\right) B\left(X_{j}, \operatorname{ad} X_{i} \operatorname{ad} X_{j}(Y)\right) \\
& =\sum_{i} \nabla_{i} u\left\{\sum_{j} B\left(X_{j}, \operatorname{ad} X_{i} \operatorname{ad} Y\left(X_{j}\right)\right)\right\} .
\end{aligned}
$$

The summation over $j$ in this last expression gives $\operatorname{tr}_{\mathfrak{g}}\left(\operatorname{ad} X_{i}\right.$ ad $\left.Y\right)$, since $\left\{X_{j}\right\}$ is an orthonormal basis. This proves the identity for $p=2$.

Assume $(1)_{p}$ is true. Proceeding as in the case $p=2$, we may write

$$
\begin{aligned}
S_{p+1}= & \sum_{i_{p+1}} \nabla_{i_{p+1}}\left\{\sum_{i_{1}, \ldots, i_{p}}\left(\nabla_{i_{1}} \cdots \nabla_{i_{p}} u\right) \operatorname{ad} X_{i_{1}} \cdots \operatorname{ad} X_{i_{p}}\left[X_{i_{p+1}}, Y\right]\right\} \\
& +\sum_{i_{1}, \ldots, i_{p}}\left(\nabla_{i_{1}} \cdots \nabla_{i_{p}} u\right)\left\{\sum_{i_{p+1}} B\left(X_{i_{p+1}}, \operatorname{ad} X_{i_{1}} \cdots \operatorname{ad} X_{i_{p}} \operatorname{ad} Y\left(X_{i_{p+1}}\right)\right)\right\} .
\end{aligned}
$$

We apply the induction hypothesis to the first group of terms to write it as a trace, and we note that the summation over $i_{p+1}$ in the second group of terms also gives a trace. Thus $S_{p+1}$ is equal to

$$
\begin{aligned}
& \sum_{i_{p+1}} \nabla_{i_{p+1}}\left\{\sum_{i_{1}, \ldots, i_{p-1}}\left(\nabla_{i_{1}} \cdots \nabla_{i_{p-1}} u\right) \operatorname{tr}_{\mathfrak{g}}\left(\operatorname{ad} X_{i_{1}} \cdots \operatorname{ad} X_{i_{p-1}} \operatorname{ad}\left[X_{i_{p+1}}, Y\right]\right)\right\} \\
& \quad+\sum_{i_{1}, \ldots, i_{p}}\left(\nabla_{i_{1}} \cdots \nabla_{i_{p}} u\right) \operatorname{tr}_{\mathfrak{g}}\left(\operatorname{ad} X_{i_{1}} \cdots \operatorname{ad} X_{i_{p}} \operatorname{ad} Y\right) .
\end{aligned}
$$


The second set of terms in this last equation gives the desired formula for $S_{p+1}$. So to complete the induction step we must prove that the first set of terms vanishes. We relabel $i_{p+1}$ as $i_{p}$ and we observe that

$$
\begin{aligned}
\operatorname{tr}_{\mathfrak{g}}\left(\operatorname{ad} X_{i_{1}} \cdots \operatorname{ad} X_{i_{p-1}} \operatorname{ad}\left[X_{i_{p}}, Y\right]\right)= & \operatorname{tr}_{\mathfrak{g}}\left(\operatorname{ad} X_{i_{1}} \cdots \operatorname{ad} X_{i_{p-1}} \operatorname{ad} X_{i_{p}} \operatorname{ad} Y\right) \\
& -\operatorname{tr}_{\mathfrak{g}}\left(\operatorname{ad} X_{i_{p}} \operatorname{ad} X_{i_{1}} \cdots \operatorname{ad} X_{i_{p-1}} \operatorname{ad} Y\right) .
\end{aligned}
$$

Since $\nabla_{i_{1}} \cdots \nabla_{i_{p}} u$ is symmetric in the indices $i_{1}, \ldots, i_{p}$, we may make an obvious change of indices to prove that

$$
\sum_{i_{1}, \ldots, i_{p}} \nabla_{i_{1}} \cdots \nabla_{i_{p}} u \operatorname{tr}_{\mathfrak{g}}\left(\operatorname{ad} X_{i_{1}} \cdots \operatorname{ad} X_{i_{p-1}} \operatorname{ad}\left[X_{i_{p}}, Y\right]\right)=0 .
$$

This completes the induction.

Let $X \in \mathfrak{g}$. We calculate the trace identities $(1)_{p}$ for the elements

$$
u=(1 /(n+p) !) I\left(X^{n+p}\right)
$$

introduced in $\S 1.1$. We have

$$
\nabla_{i_{1}} \cdots \nabla_{i_{p}} u=\frac{1}{n !} \int_{G}\left(X^{g}\right)^{n} B\left(X^{g}, X_{i_{1}}\right) \cdots B\left(X^{g}, X_{i_{p}}\right) d g .
$$

Using (2) and the expansion of $X^{g}$ relative to the orthonormal basis $\left\{X_{i}\right\}$, we obtain the identity

(3) $\frac{1}{n !} \int_{G}\left(X^{g}\right)^{n}\left(\operatorname{ad} X^{g}\right)^{p}(Y) d g=\frac{1}{(n+1) !} \int_{G}\left(X^{g}\right)^{n+1} \operatorname{tr}_{g}\left(\left(\operatorname{ad} X^{g}\right)^{p-1} \operatorname{ad} Y\right) d g$

for any $X, Y \in \mathfrak{g}$ and any integers $n \geq 0$ and $p \geq 2$.

Proposition 1.6. Suppose $\mathfrak{g}$ is simple and $B$ is the Killing form. Then for all $X, Y \in \mathfrak{g}$,

$$
\int_{G}\left(\operatorname{ad} X^{g}\right)^{2}(Y) d g=\int_{G} X^{g} B\left(X^{g}, Y\right) d g=\frac{B(X, X)}{\operatorname{dim} \mathfrak{g}} Y .
$$

Proof. The first equality in (4) follows from the definition of the Killing form and equation (3). For fixed $X$, the linear map

$$
Y \rightarrow \int_{G} X^{g} B\left(X^{g}, Y\right) d g
$$

on $\mathfrak{g}$ commutes with $\operatorname{Ad}(G)$. The adjoint representation is irreducible. By Schur's lemma the above map is a scalar multiple of the identity. The Schur orthogonality relations imply that

$$
\int_{G} B\left(X^{g}, Y\right) B\left(X^{g}, Z\right) d g=\frac{B(X, X)}{\operatorname{dim} \mathfrak{g}} B(Z, Y)
$$

for all $X, Y, Z \in \mathfrak{g}$. This proves the second equality in (4).

Proposition 1.7. If $p \geq 1$ is an odd integer, then for all integers $n \geq 0$ and all $X, Y \in \mathfrak{g}$

$$
\int_{G}\left(X^{g}\right)^{n}\left(\operatorname{ad} X^{g}\right)^{p}(Y) d g=0
$$


Proof. The case $p=1$ is Corollary 1.4 , so we may assume $p \geq 3$. First observe that for $Z \in \mathfrak{g}$, ad $Z$ is skew-symmetric relative to $B$, so

$$
\operatorname{tr}_{\mathfrak{g}}\left((\operatorname{ad} Z)^{p}\right)=0 \text {. }
$$

If we substitute $Z=X+t Y$ in (6), then the coefficient of $t$ is

$$
p \operatorname{tr}_{\mathfrak{g}}\left((\operatorname{ad} X)^{p-1} Y\right) \text {. }
$$

Thus we have

$$
\operatorname{tr}_{\mathfrak{g}}\left((\operatorname{ad} X)^{p-1} \text { ad } Y\right)=0
$$

From (7) we conclude that the right side of (3) vanishes, and hence (5) holds.

We may form a "generating function" for the identities (3) by multiplying equation (3) by $t^{n+p}$ and summing over $n \geq 0$. This gives the identity

$$
\int_{G} e^{t X^{g}}\left(\operatorname{ad} t X^{g}\right)^{p}(Y) d g=\int_{G}\left(e^{t X^{g}}-1\right) \operatorname{tr}_{\mathfrak{g}}\left(\left(\operatorname{ad} t X^{g}\right)^{p-1} \text { ad } Y\right) d g
$$

for all $X, Y \in \mathfrak{g}$ and all integers $p \geq 2$. Here we view (8) as an identity in formal power series in the variable $t$ with coefficients in $S(\mathfrak{g})$.

Suppose $\mathfrak{g}$ is semisimple. Then

$$
\int_{G}\left(\operatorname{ad} t X^{g}\right)^{p-1} d g
$$

is a scalar on each simple ideal of $\mathfrak{g}$ by Schur's lemma, and ad $Y$ has trace zero on each simple ideal. Thus

$$
\int_{G} \operatorname{tr}_{\mathfrak{g}}\left(\left(\operatorname{ad} t X^{g}\right)^{p-1} \text { ad } Y\right) d g=0
$$

in this case, so identity (8) becomes

$$
\int_{G} e^{t X^{g}}\left(\operatorname{ad} t X^{g}\right)^{p}(Y) d g=\int_{G} e^{t X^{g}} \operatorname{tr}_{\mathfrak{g}}\left(\left(\operatorname{ad} t X^{g}\right)^{p-1} \text { ad } Y\right) d g
$$

for all integers $p \geq 1$.

\section{SYMMETRIZATION AND NORMAL ORDERING}

2.1. Formal variables and intertwining operators. Let $\hat{\mathfrak{g}}$ be the (untwisted) affine algebra associated with the reductive Lie algebra $\mathfrak{g}$ and invariant bilinear form $B$. Recall that $\hat{\mathfrak{g}}$ is the central extension of the "loop algebra" $\mathfrak{g} \otimes \mathbf{C}\left[z, z^{-1}\right]$ with commutation relations

$$
\left[X \otimes z^{m}, Y \otimes z^{n}\right]=[X, Y] \otimes z^{m+n}+m \delta_{m,-n} B(X, Y) c .
$$

Here $\delta_{m, k}$ is the Kronecker symbol, and $c$ is a basis for the center of $\hat{\mathfrak{g}}$. For $X \in \mathfrak{g}$ and $n \in \mathbf{Z}$, we will write $X(n)$ for $X \otimes z^{n}$, viewed as an element of $\hat{\mathfrak{g}}$. We have the degree derivation $d_{0}$ of $\hat{\mathfrak{g}}$ which acts by

$$
d_{0} \cdot X(n)=n X(n), \quad d_{0} \cdot c=0 .
$$


Denote by $\hat{\mathfrak{g}}^{e}$ the algebra $\hat{\mathfrak{g}}$ extended by $d_{0}$.

Set $\mathfrak{u}=\mathfrak{g} \otimes \mathbf{C}[z] z$. For $k=1,2, \ldots$ define $\mathfrak{u}_{k}=\mathfrak{g} \otimes \mathbf{C}[z] z^{k}$. Then

$$
\mathfrak{u}=\mathfrak{u}_{1} \supset \mathfrak{u}_{2} \supset \cdots \quad \text { and }\left[\mathfrak{u}_{k}, \mathfrak{u}_{j}\right] \subset \mathfrak{u}_{k+j} .
$$

The Lie algebra $\mathfrak{u} / \mathfrak{u}_{k}$ is nilpotent and finite-dimensional for $k=1,2, \ldots$.

We shall use (two-sided) formal Laurent series in several indeterminates, generally following the notation in [L-W]. Thus if $\zeta$ is a formal variable and $M$ is a $\hat{\mathfrak{g}}^{e}$ module, then $M\{\zeta\}$ will denote the $\hat{\mathfrak{g}}^{e}$ module of formal Laurent series in $\zeta$ with coefficients in $M$. Set

$$
X(\zeta)=\sum_{n \in \mathbf{Z}} \zeta^{n} X(n)
$$

We view $X(\zeta)$ as an element of $\operatorname{Hom}(M, M\{\zeta\})$. Note that

$$
\left[d_{0}, X(\zeta)\right]=D X(\zeta)
$$

where $D=\zeta \partial / \partial \zeta$.

To avoid the divergent infinite sums that arise when formal Laurent series are multiplied, we use the device of adjoining additional commuting formal variables and making the natural identifications

$$
M\left\{\zeta_{1}\right\}\left\{\zeta_{2}\right\}=M\left\{\zeta_{2}\right\}\left\{\zeta_{1}\right\}=M\left\{\zeta_{1}, \zeta_{2}\right\}
$$

For example, if $X, Y \in \mathfrak{g}$ then the commutator $\left[X\left(\zeta_{1}\right), Y\left(\zeta_{2}\right)\right]$ is well defined as an element of $\operatorname{Hom}\left(M, M\left\{\zeta_{1}, \zeta_{2}\right\}\right)$. From (1) we calculate that

$$
\left[X\left(\zeta_{1}\right), Y\left(\zeta_{2}\right)\right]=\delta\left(\zeta_{1} / \zeta_{2}\right)[X, Y]\left(\zeta_{2}\right)+(D \delta)\left(\zeta_{1} / \zeta_{2}\right) B(X, Y) c
$$

where

$$
\delta(\zeta)=\sum_{n \in \mathbf{Z}} \zeta^{n}
$$

(cf. [L-W, Theorem 2.3]).

This formalism turns out to be particularly useful in studying families $\left\{T_{k}\right\}$ of operators in $\operatorname{Hom}(M, M)$ in terms of their "generating function"

$$
T(\zeta)=\sum_{k \in \mathbf{Z}} \zeta^{k} T_{k}
$$

Here $T(\zeta) \in \operatorname{Hom}(M, M\{\zeta\})$. Call $T_{k}$ the $\zeta$-homogeneous component of degree $k$ in $T(\zeta)$.

Lemma 2.1. Let $T(\zeta) \in \operatorname{Hom}(M, M\{\zeta\})$ be given by (4). The following are equivalent:

(i) For all $k \in \mathbf{Z}, T_{k}$ commutes with the action of $\hat{\mathfrak{g}}$ on $M$.

(ii) For all $X \in \mathfrak{g},\left[T\left(\zeta_{1}\right), X\left(\zeta_{2}\right)\right]=0$ as an element of $\operatorname{Hom}\left(M, M\left\{\zeta_{1}, \zeta_{2}\right\}\right)$.

Proof. Since $\zeta_{1}$ and $\zeta_{2}$ are commuting variables, we have

$$
\left[T\left(\zeta_{1}\right), X\left(\zeta_{2}\right)\right]=\sum_{k, n} \zeta_{1}^{k} \zeta_{2}^{n}\left[T_{k}, X(n)\right]
$$


But the elements $X(n)$, for $X \in \mathfrak{g}$ and $n \in \mathbf{Z}$, generate $\hat{\mathfrak{g}}$ as a Lie algebra. Thus the left side of (5) is zero as a formal series if and only if all the operators $T_{k}$ commute with $\hat{\mathfrak{g}}$.

2.2. The category $\mathscr{F}$ and normal ordering. We denote by $\mathscr{F}$ (for "formal") the category of all $\hat{\mathfrak{g}}^{e}$ modules $M$ such that for all $v \in M$, there exists an integer $k$ such that

$$
\mathfrak{u}_{k} v=0 .
$$

For $X \in \mathfrak{g}$ define $X_{ \pm}(\zeta) \in \hat{\mathfrak{g}}\{\zeta\}$ by

$$
X_{-}\{\zeta\}=\sum_{n<0} \zeta^{n} X(n), \quad X_{+}(\zeta)=\sum_{n \geq 0} \zeta^{n} X(n) .
$$

Note that $X(\zeta)=X_{-}(\zeta)+X_{+}(\zeta)$. If $M$ is in the category $\mathscr{F}$, then $X_{+}(\zeta) v$ is a polynomial in $\zeta$ for each $v \in M$. We shall use this decomposition to form normal-ordered powers of $X(\zeta)$ as operators from $M$ to $M\{\zeta\}$. It is convenient to introduce the following class of formal operators, which includes the operators $X(\zeta)$.

Let $\operatorname{Hom}_{\infty}(M, M\{\zeta\})$ to be the linear space of all formal operator series

$$
T(\zeta)=\sum_{n \in \mathbf{Z}} \zeta^{n} T_{n}, \quad \text { with } T_{n} \in \operatorname{Hom}(M, M),
$$

such that for every vector $v \in M, T_{n} v=0$ for all sufficiently large $n$. We can express this by saying that the series $T(\zeta) v$ is formally meromorphic at $\zeta=\infty$, since it has only a finite number of nonzero positive powers of $\zeta$. Define

$$
T_{+}(\zeta)=\sum_{n \geq 0} \zeta^{n} T_{n}, \quad T_{-}(\zeta)=\sum_{n<0} \zeta^{n} T_{n} .
$$

If $T(\zeta)$ is in $\operatorname{Hom}_{\infty}(M, M\{\zeta\})$ then obviously so are $T_{ \pm}(\zeta)$ and $D T(\zeta)$, where as usual $D=\zeta \partial / \partial \zeta$.

Lemma 2.2. Assume that $M$ is a $\hat{\mathfrak{g}}^{e}$ module in the category $\mathscr{F}$. Let $X \in \mathfrak{g}$ and $T(\zeta) \in \operatorname{Hom}_{\infty}(M, M\{\zeta\})$. Then the formal products $X_{-}(\zeta) T(\zeta)$ and $T(\zeta) X_{+}(\zeta)$ exist as elements of $\operatorname{Hom}_{\infty}(M, M\{\zeta\})$.

Proof. Let $v \in M$. Then there are integers $m(v)$ and $n(v)$ such that $X(j) v=$ 0 for $j>m(v)$ and $T_{k} v=0$ for $k>n(v)$. Set $p=m(v)+n(v)$. Then we can write the formal expansion of $T(\zeta) X_{+}(\zeta) v$ as

$$
\sum_{m \leq p} \zeta^{m}\left\{\sum_{k=m-p}^{m} T_{k} X_{+}(m-k) v\right\} .
$$

Note that the sum in braces is over a finite range of the index $k$, so it is defined in $M$. Since the whole series is truncated above at $p=p(v)$, the linear transformation given by this expansion is in $\operatorname{Hom}_{\infty}(M, M\{\zeta\})$. A similar calculation applies to the formal expansion of $X_{-}(\zeta) T(\zeta) v$.

We can now define the normal-ordered symmetrization map from $S(\mathfrak{g})$ to operators on $M$, using the formal variable machinery. 
Lemma 2.3. Let $M$ be a module in the category $\mathscr{F}$. There exists a unique linear map

$$
\sigma: S(\mathfrak{g}) \rightarrow \operatorname{Hom}_{\infty}(M, M\{\zeta\})
$$

such that for $m \in M, X \in \mathfrak{g}$, and all integers $n \geq 0$

$$
\sigma\left(X^{n}\right)(\zeta) m=\sum_{k=0}^{n}\left(\begin{array}{l}
n \\
k
\end{array}\right) X_{-}(\zeta)^{k} X_{+}(\zeta)^{n-k} m,
$$

where $\left(\begin{array}{l}n \\ k\end{array}\right)$ denotes the usual binomial coefficient.

Proof. By Lemma 2.2, the right side of (2) is in $\operatorname{Hom}_{\infty}(M, M\{\zeta\})$ and uniquely determines $\sigma$, since the elements $\left\{X^{n}\right\}$ span $S^{n}(\mathfrak{g})$.

To prove the existence of $\sigma$, choose a basis $X_{1}, \ldots, X_{r}$ for $\mathfrak{g}$. The ordered monomials

$$
X^{\alpha}=X_{1}^{\alpha_{1}} \cdots X_{r}^{\alpha_{r}}
$$

form a basis for $S^{n}(\mathfrak{g})$, where $n=|\alpha|=\alpha_{1}+\cdots+\alpha_{r}$. We define $\sigma\left(X^{\alpha}\right)(\zeta)$ as follows:

Let $t \in \mathbf{C}^{r}$ and set $X=t_{1} X_{1}+\cdots+t_{r} X_{r}$. For every $\alpha \in \mathbf{N}^{r}$ with $|\alpha|=n$, define $\Xi_{ \pm}(\alpha, \zeta) \in \operatorname{Hom}_{\infty}(M, M\{\zeta\})$ to be the coefficients of $t^{\alpha} / \alpha$ ! in the expansions

$$
\frac{1}{n !} X_{ \pm}(\zeta)^{n}=\sum_{|\alpha|=n} \frac{t^{\alpha}}{\alpha !} \Xi_{ \pm}(\alpha, \zeta)
$$

(same choice of \pm on each side of the equation). Note that the left side of (3) is already defined, by Lemma 2.2. Now we define

$$
\frac{1}{\alpha !} \sigma\left(X^{\alpha}\right)(\zeta)=\sum_{\beta+\gamma=\alpha} \frac{1}{\beta ! \gamma !} \Xi_{-}(\beta, \zeta) \Xi_{+}(\gamma, \zeta) .
$$

The operator products on the right are defined because of Lemma 2.2. We extend $\sigma$ by linearity to $S(\mathfrak{g})$. It is clear from the multinomial expansion in $S(\mathfrak{g})$ that equation (2) is satisfied, and hence $\sigma$ is independent of the choice of basis.

Lemma 2.4. Let $Y \in \mathfrak{g}$ and $u \in S(\mathfrak{g})$. Then

$$
[Y, \sigma(u)(\zeta)]=\sigma(\operatorname{ad} Y(u))(\zeta)
$$

as an element of $\operatorname{Hom}(M, M\{\zeta\})$.

Proof. It suffices to prove this when $u=X^{n}$, for all $X \in \mathfrak{g}$. Since $\sigma(X)(\zeta)=$ $X(\zeta)$, the intertwining property holds when $n=1$. Also from the relation

$$
\left(\begin{array}{c}
n+1 \\
k
\end{array}\right)=\left(\begin{array}{c}
n \\
k-1
\end{array}\right)+\left(\begin{array}{l}
n \\
k
\end{array}\right)
$$

and the expansion (2) it follows that

$$
\sigma\left(X^{n+1}\right)(\zeta)=X_{-}(\zeta) \sigma\left(X^{n}\right)(\zeta)+\sigma\left(X^{n}\right)(\zeta) X_{+}(\zeta)
$$


From the commutation relations in $\hat{\mathfrak{g}}$ one has

$$
\left[Y, X_{ \pm}(\zeta)\right]=[Y, X]_{ \pm}(\zeta)
$$

for all $X$ in $\mathfrak{g}$. The lemma now follows from these last two relations by induction on $n$, using the fact that ad $Y$ is a derivation of $S(\mathfrak{g})$.

For $X \in \mathfrak{g}$, we define the normal-ordered power

$$
: X(\zeta)^{n}:=\sigma\left(X^{n}\right)(\zeta)
$$

as an element of $\operatorname{Hom}_{\infty}(M, M\{\zeta\})$. Equation (2) shows how to calculate this power by the binomial expansion, as if $X_{-}(\zeta)$ and $X_{+}(\zeta)$ were commuting operators.

We adjoin another formal variable $t$ and for $X \in \mathfrak{g}$ we form the generating function

$$
e^{t X}=\sum_{n=0}^{\infty} \frac{t^{n}}{n !} X^{n},
$$

which we view as an element of $S(\mathfrak{g})\{t\}$. In this case the degree of homogeneity relative to $t$ is the usual degree in $S(\mathfrak{g})$. We extend the map $\sigma$ to a C-linear map from $S(\mathfrak{g})\{t\}$ to $\operatorname{Hom}(M, M\{\zeta, t\})$. With the normal-order notation of (5), we can write

$$
\sigma\left(e^{t X}\right)(\zeta)=\sum_{n=0}^{\infty} \frac{t^{n}}{n !}: X(\zeta)^{n}:,
$$

which we also denote as $: e^{t X(\zeta)}:$. From the ordered binomial expansion (2) we obtain the formula

$$
: e^{t X(\zeta)}:=e^{t X_{-}(\zeta)} e^{t X_{+}(\zeta)}
$$

for $X \in \mathfrak{g}$. Equation (6) uniquely determines the normal ordered map $\sigma$.

2.3. Symmetrization. Let Symm denote the symmetrization operation on the free associative algebra with generators $x, y$. For a monomial of degree 1 in $y$, we have

$$
\operatorname{Symm}\left(x^{n} y\right)=\frac{1}{n+1}\left(x^{n} y+x^{n-1} y x+\cdots+x y x^{n-1}+y x^{n}\right) .
$$

We define $\operatorname{Symm}\left(e^{x} y\right)$ to be the formal sum of the symmetrizations of the homogeneous components. We can then specialize $x$ and $y$ to be any elements in an associative algebra.

Lemma 2.5. Let $X, Y \in \mathfrak{g}$ and let $M$ be a module in the category $\mathscr{F}$. Then for every positive integer $n$,

$$
\begin{aligned}
\sigma\left(X^{n} Y\right)(\zeta)=\sum_{k=0}^{n}\left(\begin{array}{l}
n \\
k
\end{array}\right)\left\{X_{-}(\zeta)^{k}\right. & \operatorname{Symm}\left(Y_{+}(\zeta) X_{+}(\zeta)^{n-k}\right) \\
& \left.+\operatorname{Symm}\left(Y_{-}(\zeta) X_{-}(\zeta)^{k}\right) X_{+}(\zeta)^{n-k}\right\}
\end{aligned}
$$

as operators from $M$ to $M\{\zeta\}$. 
Proof. Introduce a formal variable $t$. Then $(n+1) \sigma\left(X^{n} Y\right)(\zeta)$ is the coeffcient of $t$ in $\sigma\left((X+t Y)^{n+1}\right)(\zeta)$. On the other hand, by the ordered binomial expansion (equation (2) in $\S 2.2$ ), we have

$$
\sigma\left((X+t Y)^{n+1}\right)(\zeta)=\sum_{k=0}^{n+1}\left(\begin{array}{c}
n+1 \\
k
\end{array}\right)\left(X_{-}(\zeta)+t Y_{-}(\zeta)\right)^{k}\left(X_{+}(\zeta)+t Y_{+}(\zeta)\right)^{n+1-k}
$$

The coefficient of $t$ on the right is

$$
\begin{aligned}
\sum_{k=0}^{n}(k+1)\left(\begin{array}{l}
n+1 \\
k+1
\end{array}\right) \operatorname{Symm}\left(Y_{-}(\zeta) X_{-}(\zeta)^{k}\right) X_{+}(\zeta)^{n-k} \\
\quad+\sum_{k=0}^{n}(n+1-k)\left(\begin{array}{c}
n+1 \\
k
\end{array}\right) X_{-}(\zeta)^{k} \operatorname{Symm}\left(Y_{+}(\zeta) X_{+}(\zeta)^{n-k}\right) .
\end{aligned}
$$

Now we use the identities $\left(\begin{array}{c}n+1 \\ k+1\end{array}\right)(k+1)=\left(\begin{array}{c}n+1 \\ k\end{array}\right)(n+1-k)=\left(\begin{array}{l}n \\ k\end{array}\right)(n+1)$ to obtain (1).

Example. Let $X, Y \in \mathfrak{g}$. From (1) we see that

$$
\begin{aligned}
\sigma(X Y)(\zeta)= & \frac{1}{2}\left\{X_{-}(\zeta) Y_{-}(\zeta)+Y_{-}(\zeta) X_{-}(\zeta)\right\}+X_{-}(\zeta) Y_{+}(\zeta)+Y_{-}(\zeta) X_{+}(\zeta) \\
& +\frac{1}{2}\left\{X_{+}(\zeta) Y_{+}(\zeta)+Y_{+}(\zeta) X_{+}(\zeta)\right\}
\end{aligned}
$$

Introducing a formal variable $t$, we can express the identities (1) in generating function form as follows:

Corollary 2.6. Let $X, Y \in \mathfrak{g}$. Then

$$
\sigma\left(e^{t X} Y\right)(\zeta)=\operatorname{Symm}\left(Y_{-}(\zeta) e^{t X_{-}(\zeta)}\right) e^{t X_{+}(\zeta)}+e^{t X_{-}(\zeta)} \operatorname{Symm}\left(Y_{+}(\zeta) e^{t X_{+}(\zeta)}\right) .
$$

For the calculation of commutation relations, we shall need to apply the symmetrization operator that appears in formulas (1) and (2) with $Y(\zeta)$ replaced by operators such as $D^{p} Y(\zeta)$. We observe that if $X \in \mathfrak{g}$ and $T(\zeta) \in$ $\operatorname{Hom}_{\infty}(M, M\{\zeta\})$, then the operator

(3) $\sum_{k=0}^{n}\left(\begin{array}{l}n \\ k\end{array}\right)\left\{X_{-}(\zeta)^{k} \operatorname{Symm}\left(T_{+}(\zeta) X_{+}(\zeta)^{n-k}\right)+\operatorname{Symm}\left(T_{-}(\zeta) X_{-}(\zeta)^{k}\right) X_{+}(\zeta)^{n-k}\right\}$

is defined in $\operatorname{Hom}_{\infty}(M, M\{\zeta\})$.

We denote the operator in (3) as $\sigma\left(X(\zeta)^{n}, T(\zeta)\right)$. When $T(\zeta)=Y(\zeta)$, for some $Y \in \mathfrak{g}$, then

$$
\sigma\left(X(\zeta)^{n}, T(\zeta)\right)=\sigma\left(X^{n} Y\right)(\zeta)
$$

by Lemma 2.5 , where the product $X^{n} Y$ is calculated in $S(\mathfrak{g})$. Extending formula (2) to this more general context, we define

$$
\sigma\left(e^{t X(\zeta)}, T(\zeta)\right)=\sum_{t=0}^{\infty} \frac{t^{n}}{n !} \sigma\left(X(\zeta)^{n}, T(\zeta)\right)
$$


with the series on the right being understood as a formal series in $t$. We can also write this definition as

(4) $\sigma\left(e^{t X(\zeta)}, T(\zeta)\right)=\operatorname{Symm}\left(T_{-}(\zeta) e^{t X_{-}(\zeta)}\right) e^{t X_{+}(\zeta)}+e^{t X_{-}(\zeta)} \operatorname{Symm}\left(T_{+}(\zeta) e^{t X_{+}(\zeta)}\right)$.

The map $T(\zeta) \rightarrow \sigma\left(e^{t X(\zeta)}, T(\zeta)\right)$ is linear and commutes with the action of ad $X$, for $X \in \mathfrak{g}$.

\section{SUgAwARA FIELDS}

3.1. Commutation relations. Let $u \in S(\mathfrak{g})^{G}$. The formal operator $\sigma(u)(\zeta)$ is defined on every module $M$ in the category $\mathscr{F}$, and we shall call it the Sugawara field associated with $u$ (cf. Introduction for the motivation for this terminology). To obtain Sugawara fields, we can take $X \in \mathfrak{g}$ and project $X^{k}$ onto the $G$-invariants by integrating over $G$. We set

$$
u_{k}=\int_{G}\left(X^{g}\right)^{k} d g
$$

in $S(\mathfrak{g})^{G}$, for $k=2,3, \ldots$. The Sugawara field associated with $u_{k}$ can then be written formally as

$$
\sigma\left(u_{k}\right)(\zeta)=\int_{G}: X^{g}(\zeta)^{k}: d g
$$

For fixed $v \in M$, every $\zeta$-homogeneous component of the function $g \rightarrow$ $: X^{g}(\zeta)^{k}: v$ lies in a finite-dimensional subspace of $M$ independent of $g$. Thus the integral on the right side of (1) exists as the formal sum of its $\zeta$ homogeneous components and obviously commutes with the action of $\mathfrak{g}$ on $M$.

We shall calculate commutation relations between a Sugawara field $\sigma(u)\left(\zeta_{1}\right)$ and $Y\left(\zeta_{2}\right)$, with $Y \in \mathfrak{g}$. The map $u \rightarrow \sigma(u)\left(\zeta_{1}\right)$ is linear and invariants of the form $u_{k}$ as above span $S^{k}(\mathfrak{g})^{G}$, as $X$ ranges over $\mathfrak{g}$. Thus we only need to calculate the commutators

$$
\left[\sigma\left(u_{k}\right)\left(\zeta_{1}\right), Y\left(\zeta_{2}\right)\right]
$$

for $k=2,3, \ldots$.

Fix $X \in \mathfrak{g}$. It is convenient to introduce the exponential generating function for the invariants $u_{k}$ obtained from powers of $X$. Let $t$ be a formal variable, and define

$$
u(t)=\int_{G} e^{t X^{g}} d g=\sum_{k=0}^{\infty} \frac{t^{k}}{k !} u_{k} .
$$

We view $u(t)$ as a formal series in $t$ with coefficients in $S(\mathfrak{g})^{G}$, as in $\S 1.2$. Consider the operator

$$
\sigma(u(t))(\zeta)=\int_{G}: e^{t X^{g}(\zeta)}: d g
$$


on $M$. By the remarks made earlier, the integral on the right side of (2) exists as the formal sum of its $t$-homogeneous components and obviously commutes with the action of $\mathfrak{g}$ on $M$.

Our first goal is to determine the general form of the commutator of $Y\left(\zeta_{2}\right)$ with the normal-ordered exponential appearing in the integrand in (2), when $Y \in \mathfrak{g}$. For this we will need a polarized version of the commutation relations in $\hat{\mathfrak{g}}$. Decompose the delta function $\delta(\zeta)=\delta_{+}(\zeta)+\delta_{-}(\zeta)$, where

$$
\delta_{+}(\zeta)=\sum_{n \geq 0} \zeta^{n}, \quad \delta_{-}(\zeta)=\sum_{n<0} \zeta^{n}
$$

Let $\delta^{(k)}(\zeta)=(d / d \zeta)^{k} \delta(\zeta)$ with a similar meaning for $\delta_{ \pm}^{(k)}(\zeta)$.

Lemma 3.1. Let $X, Y \in \mathfrak{g}$. Then

$$
\left[X_{ \pm}\left(\zeta_{1}\right), Y\left(\zeta_{2}\right)\right]=\delta_{ \pm}\left(\zeta_{1} / \zeta_{2}\right)[X, Y]\left(\zeta_{2}\right)+\left(D \delta_{ \pm}\right)\left(\zeta_{1} / \zeta_{2}\right) B(X, Y) c .
$$

Furthermore, for $n \geq 2$ one has

$$
\left(\operatorname{ad} X_{ \pm}\left(\zeta_{1}\right)\right)^{n}\left(Y\left(\zeta_{2}\right)\right)=\frac{( \pm 1)^{n-1}}{(n-1) !} \delta_{ \pm}^{(n-1)}\left(\zeta_{1} / \zeta_{2}\right)\left((\operatorname{ad} X)^{n}(Y)\right)\left(\zeta_{2}\right)
$$

Proof. Equation (3) follows directly from equation (3) of §2.1. Taking another commutator and using the fact that $c$ is central, we get

$$
\left(\operatorname{ad} X_{ \pm}\left(\zeta_{1}\right)\right)^{2} Y\left(\zeta_{2}\right)=\delta_{ \pm}\left(\zeta_{1} / \zeta_{2}\right)^{2}\left((\operatorname{ad} X)^{2} Y\right)\left(\zeta_{2}\right)+(D \delta)_{ \pm}\left(\zeta_{1} / \zeta_{2}\right) B(X,[X, Y]) c
$$

But $B(X,[X, Y])=-B([X, X], Y)=0$, so the $c$-term vanishes. Iterating this calculation, we find that

$$
\left(\operatorname{ad} X_{ \pm}\left(\zeta_{1}\right)\right)^{n} Y\left(\zeta_{2}\right)=\delta_{ \pm}\left(\zeta_{1} / \zeta_{2}\right)^{n}\left((\operatorname{ad} X)^{n}(Y)\right)\left(\zeta_{2}\right)
$$

for $n \geq 2$. Now observe that $\delta_{+}(\zeta)=(1-\zeta)^{-1}$ in $C[[\zeta]]$, so

$$
\delta_{+}(\zeta)^{n}=(1-\zeta)^{-n}=(1 /(n-1) !) \delta_{+}^{(n)}(\zeta)
$$

in $\mathbf{C}[[\zeta]]$. Similarly, $\delta_{-}(\zeta)=\zeta^{-1}\left(1-\zeta^{-1}\right)^{-1}$ in $\mathbf{C}\left[\left[\zeta^{-1}\right]\right]$, so

$$
\delta_{-}(\zeta)^{n}=(\zeta-1)^{-n}=\left((-1)^{n-1} /(n-1) !\right) \delta_{-}^{(n)}(\zeta)
$$

in $\mathbf{C}\left[\left[\zeta^{-1}\right]\right]$. Using these formulas in (5), we obtain (4).

We can now obtain a preliminary version of the commutator formula.

Proposition 3.2. Let $X, Y \in \mathfrak{g}$. Then on any module in the category $\mathscr{F}$,

(6) $\left[: e^{t X\left(\zeta_{1}\right)}:, Y\left(\zeta_{2}\right)\right]=c(D \delta)\left(\zeta_{1} / \zeta_{2}\right) B(t X, Y): e^{t X\left(\zeta_{1}\right)}:$

$$
+\left\{\sum_{n \geq 1} \frac{\delta^{(n-1)}\left(\zeta_{1} / \zeta_{2}\right)}{(n-1) !} \frac{(\operatorname{ad} t X)^{n}}{n !}\right\} e^{t X_{-}\left(\zeta_{1}\right)} Y\left(\zeta_{2}\right) e^{t X_{+}\left(\zeta_{1}\right)} .
$$


Proof. Let $R, S, T$ be noncommuting variables in a free associative algebra. Then one has the formal identity

$$
\begin{aligned}
{\left[e^{R} e^{S}, T\right] } & =e^{R}\left\{e^{S} T e^{-S}-e^{-R} T e^{R}\right\} e^{S} \\
& =e^{R}\left\{e^{\operatorname{ad} S} T-e^{-\operatorname{ad} R} T\right\} e^{S} \\
& =e^{R}\left\{\sum_{n \geq 1} \frac{1}{n !}\left((\operatorname{ad} S)^{n} T-(-\operatorname{ad} R)^{n} T\right)\right\} e^{S},
\end{aligned}
$$

where $e^{R}$ is defined by the formal exponential series and ad $R(T)=R T-T R$.

To obtain (6), we write $: e^{t X\left(\zeta_{1}\right)}:=e^{t X_{-}\left(\zeta_{1}\right)} e^{t X_{+}\left(\zeta_{1}\right)}$ by formula $(6)$ of $\S 2.2$. Then we specialize (7) with $R=t X_{-}\left(\zeta_{1}\right), S=t X_{+}\left(\zeta_{1}\right)$, and $T=Y\left(\zeta_{2}\right)$, and use Lemma 3.1.

We can use $\delta$-function identities to simplify (6). Suppose

$$
F\left(\zeta_{1}, \zeta_{2}\right)=\sum_{m, n \in \mathbf{Z}} a_{m n} \zeta_{1}^{m} \zeta_{2}^{n}
$$

is in $M\left\{\zeta_{1}, \zeta_{2}\right\}$. We say that $F$ is supported on a set $S \subset \mathbf{Z}^{2}$ if $a_{m n}=0$ when $(m, n) \notin S$. For example, if $X \in \mathfrak{g}, T(\zeta) \in \operatorname{Hom}_{\infty}(M, M\{\zeta\})$, and $v \in M$, then

$$
F\left(\zeta_{1}, \zeta_{2}\right)=e^{t X_{-}\left(\zeta_{1}\right)} T\left(\zeta_{2}\right) e^{t X_{+}\left(\zeta_{1}\right)} v
$$

is supported on a quadrant $\{m \leq r$ and $n \leq r\}$ for some integer $r$ (depending on the vector $v$ ).

Lemma 3.3. Let $F \in M\left(\zeta_{1}, \zeta_{2}\right)$ and suppose that $F$ is supported on a quadrant $\{(m, n): m \leq r$ and $n \leq r\}$. Then

$$
\delta\left(\zeta_{1} / \zeta_{2}\right) F\left(\zeta_{1}, \zeta_{2}\right)=\delta\left(\zeta_{1} / \zeta_{2}\right) F\left(\zeta_{1}, \zeta_{1}\right)
$$

$$
\delta^{\prime}\left(\zeta_{1} / \zeta_{2}\right) F\left(\zeta_{1}, \zeta_{2}\right)=D \delta\left(\zeta_{1} / \zeta_{2}\right) F\left(\zeta_{1}, \zeta_{1}\right)+\delta\left(\zeta_{1} / \zeta_{2}\right)\left(F+D_{2} F\right)\left(\zeta_{1}, \zeta_{1}\right)
$$

For any positive integer $k, \delta^{(k)}\left(\zeta_{1} / \zeta_{2}\right) F\left(\zeta_{1}, \zeta_{2}\right)$ is in the linear span of

$$
D^{p} \delta\left(\zeta_{1} / \zeta_{2}\right) D_{2}^{q} F\left(\zeta_{1}, \zeta_{1}\right)
$$

with $p+q \leq k$. Here $D_{2}=\zeta_{2} \partial / \partial \zeta_{2}$.

Proof (cf. [L-W, Proposition 3.9]). We use the notation $(x)_{[n]}$ to denote the falling factorial

$$
(x)_{[n]}=x(x-1) \cdots(x-n+1)
$$

for $n \in \mathbf{N}$ and $x \in \mathbf{C}$, with the convention that $(x)_{[0]}=1$. We have

$$
\begin{aligned}
\delta^{(k)}\left(\zeta_{1} / \zeta_{2}\right) F\left(\zeta_{1}, \zeta_{2}\right) & =\sum_{m, n \in \mathbf{Z}}\left\{\sum_{j \in \mathbf{Z}}(k+j)_{[k]}\left(\zeta_{1} / \zeta_{2}\right)^{j}\right\} a_{m n} \zeta_{1}^{m} \zeta_{2}^{n} \\
& =\sum_{m, n \in \mathbf{Z}}\left\{\sum_{j \in \mathbf{Z}}(k+n+j)_{[k]}\left(\zeta_{1} / \zeta_{2}\right)^{j}\right\} a_{m n} \zeta_{1}^{m} \zeta_{1}^{n} .
\end{aligned}
$$


Here we have interchanged the order of summation and made an index shift $j \rightarrow j+n$. Using the same argument as in Lemma 2.2, we check that these manipulations are justified at the formal series level because of the support condition on the coefficients $\left\{a_{m n}\right\}$. Taking $k=0$ and $k=1$ in this formula then yields (9) and (10).

In general we can expand the falling factorial

$$
\begin{aligned}
(k+n+j)_{[k]} & =(k+n+j)(k-1+n+j) \cdots(1+n+j) \\
& =\sum_{p+q \leq k} C_{k}(p, q) j^{p} n^{q}
\end{aligned}
$$

where the coefficients $C_{k}(p, q)$ are products of binomial coefficients and Stirling numbers of the first kind. Substituting this in the previous formula, we obtain

$$
\delta^{(k)}\left(\zeta_{1} / \zeta_{2}\right) F\left(\zeta_{1}, \zeta_{2}\right)=\sum_{p+q \leq k} C_{k}(p, q) D^{p} \delta\left(\zeta_{1} / \zeta_{2}\right) D_{2}^{q} F\left(\zeta_{1}, \zeta_{1}\right)
$$

as claimed in the lemma.

Now we apply Lemma 3.3 to $F\left(\zeta_{1}, \zeta_{2}\right)$ given in (8), noting that

$$
\left(D_{2}^{q} F\right)\left(\zeta_{1}, \zeta_{1}\right)=e^{t X_{-}\left(\zeta_{1}\right)} D^{q} T\left(\zeta_{1}\right) e^{t X_{+}\left(\zeta_{1}\right)} v .
$$

Using this in the commutator formula (6) with $T(\zeta)=Y(\zeta)$, we obtain the following:

Corollary 3.4. Let $X, Y \in \mathfrak{g}$. Then

$$
\begin{aligned}
{\left[: e^{t X\left(\zeta_{1}\right)}:, Y\left(\zeta_{2}\right)\right]=} & c(D \delta)\left(\zeta_{1} / \zeta_{2}\right) B(t X, Y): e^{t X\left(\zeta_{1}\right)}: \\
& +(\operatorname{ad} t X) \delta\left(\zeta_{1} / \zeta_{2}\right) e^{t X_{-}\left(\zeta_{1}\right)} Y\left(\zeta_{1}\right) e^{t X_{+}\left(\zeta_{1}\right)} \\
& +\frac{(\operatorname{ad} t X)^{2}}{2} \delta\left(\zeta_{1} / \zeta_{2}\right) e^{t X_{-}\left(\zeta_{1}\right)}\left\{(D+1) Y\left(\zeta_{1}\right)\right\} e^{t X_{+}\left(\zeta_{1}\right)} \\
& +\frac{(\operatorname{ad} t X)^{2}}{2} D \delta\left(\zeta_{1} / \zeta_{2}\right) e^{t X_{-}\left(\zeta_{1}\right)} Y\left(\zeta_{1}\right) e^{t X_{+}\left(\zeta_{1}\right)} \\
& +R_{3}(t X, Y)\left(\zeta_{1}, \zeta_{2}\right)
\end{aligned}
$$

where $R_{3}(t X, Y)\left(\zeta_{1}, \zeta_{2}\right)$ is a formal linear combination of terms of the form

$$
t^{m+p+q}(\operatorname{ad} X)^{m}\left(D^{j} \delta\right)\left(\zeta_{1} / \zeta_{2}\right) X_{-}\left(\zeta_{1}\right)^{p} D^{k} Y\left(\zeta_{1}\right) X_{+}\left(\zeta_{1}\right)^{q}
$$

with $m \geq 3, j+k \leq m-1$, and $p, q \geq 0$. For fixed degree $m+p+q$ in $t$, only a finite number of such terms occur.

3.2. Symmetrization identities. The next step in analyzing the commutator formula (12) of $\$ 3.1$ is to express the operator $e^{t X_{-}(\zeta)} Y(\zeta) e^{t X_{+}(\zeta)}$ in "symmetrized" form. For this we split $Y(\zeta)=Y_{+}(\zeta)+Y_{-}(\zeta)$ and use the following result:

Lemma 3.5. Let $X, Y \in \mathfrak{g}$. Then

$$
\left(\operatorname{ad} X_{ \pm}(\zeta)\right)^{n} Y_{ \pm}(\zeta)=( \pm \operatorname{ad} X)^{n} L_{n} Y_{ \pm}(\zeta)
$$


Here the \pm signs are taken the same in all terms and $L_{n}$ is the nth order differential operator

$$
L_{n}=\left(\begin{array}{c}
D+n \\
n
\end{array}\right)=\frac{(D+n)(D+n-1) \cdots(D+1)}{n !}
$$

where $D=\zeta \partial / \partial \zeta$.

Proof. We may calculate the commutation relations in the loop algebra, since no $c$-terms arise. Note that

$$
X_{+}(\zeta)=X \otimes(1-\zeta z)^{-1}
$$

in $\mathfrak{g} \otimes \mathbf{C}[[z, \zeta]]$. Thus

$$
\left(\operatorname{ad} X_{+}(\zeta)\right)^{n} Y_{+}(\zeta)=(\operatorname{ad} X)^{n} Y \otimes(1-\zeta z)^{-n-1}
$$

Now we use the binominal expansion to write

$$
Y \otimes(1-\zeta z)^{-n-1}=\sum_{k=0}^{\infty}\left(\begin{array}{c}
-n-1 \\
k
\end{array}\right)(-\zeta)^{k} Y(k) .
$$

We can write the negative binomial coefficient in (3) as

$$
\left(\begin{array}{c}
-n-1 \\
k
\end{array}\right)(-1)^{k}=\left(\begin{array}{c}
k+n \\
n
\end{array}\right)
$$

Thus from (2) and (3) we have

$$
\left(\operatorname{ad} X_{+}(\zeta)\right)^{n} Y_{+}(\zeta)=(\operatorname{ad} X)^{n}\left\{\sum_{k=0}^{\infty}\left(\begin{array}{c}
k+n \\
n
\end{array}\right) \zeta^{k} Y(k)\right\}
$$

which is formula (1) in the $(+)$ case.

The proof of the (-) case is similar, using the formula

$$
X_{-}(\zeta)=X \otimes(\zeta z)^{-1}\left(1-(\zeta z)^{-1}\right)^{-1}
$$

in $\mathfrak{g} \otimes \mathbf{C}\left[\left[z^{-1}, \zeta^{-1}\right]\right]$. We leave the details to the reader.

Let the Bernoulli numbers $b_{n}$ be defined by the expansion

$$
\frac{z}{e^{z}-1}=\sum_{n=0}^{\infty} b_{n} \frac{z^{n}}{n !} \text {. }
$$

We have the following symmetrization identity, where the differential operator $L_{n}$ is as in Lemma 3.5 and the symmetrization operator $\sigma$ is defined as in formula (4) of $\S 2.3$ :

Proposition 3.6. Let $X, Y \in \mathfrak{g}$. Then

$$
e^{t X_{-}(\zeta)} Y(\zeta) e^{t X_{+}(\zeta)}=\sum_{n=0}^{\infty} \frac{b_{n}}{n !} \sigma\left(e^{t X(\zeta)},(\operatorname{ad} t X)^{n} L_{n} Y(\zeta)\right)
$$


Proof. Let $x$ and $y$ generate a free associative algebra. Then one has the formal symmetrization identities

$$
\begin{aligned}
y e^{x} & =\sum_{n=0}^{\infty} b_{n} \frac{(\operatorname{ad} x)^{n}}{n !} \operatorname{Symm}\left(y e^{x}\right) \\
e^{x} y & =\sum_{n=0}^{\infty} b_{n} \frac{(-\operatorname{ad} x)^{n}}{n !} \operatorname{Symm}\left(y e^{x}\right)
\end{aligned}
$$

(cf. [Goo, Chapter 2, §2.1]). Splitting $Y(\zeta)=Y_{+}(\zeta)+Y_{-}(\zeta)$, we write the left side of (4) as the sum of two terms. For the term involving $Y_{+}(\zeta)$, we apply identity (5) with the specializations $x \rightarrow t X_{+}(\zeta)$ and $y \rightarrow Y_{+}(\zeta)$ and we use Lemma 3.5 to evaluate the commutators. Similarly, for the term involving $Y_{-}(\zeta)$ we apply identity (6) with the specializations $x \rightarrow t X_{-}(\zeta)$ and $y \rightarrow$ $Y_{-}(\zeta)$. We can then combine the terms using formula (4) of $\S 2.3$ and noting that $X$ commutes with $X_{ \pm}(\zeta)$.

Using the values $b_{0}=1, b_{1}=-\frac{1}{2}$ of the Bernoulli numbers and the formula $L_{1}=D+1$, we can write (4) as follows.

Corollary 3.7. Let $X, Y \in \mathfrak{g}$. Then

$$
\begin{aligned}
e^{t X_{-}(\zeta)} Y(\zeta) e^{t X_{+}(\zeta)}= & \sigma\left(e^{t X} Y\right)(\zeta)-\frac{\operatorname{ad} t X}{2} e^{t X_{-}(\zeta)}\{(D+1) Y(\zeta)\} e^{t X_{+}(\zeta)} \\
& +S(t X, Y)(\zeta)
\end{aligned}
$$

Here the remainder $S(t X, Y)(\zeta)$ is a formal linear sum of terms

$$
t^{n} S_{n}(X, Y)(\zeta)
$$

with $n \geq 2$. Furthermore, $S_{n}(X, Y)(\zeta)$ is a finite linear combination of terms of the form

$$
X_{-}(\zeta)^{p}\left\{(\operatorname{ad} X)^{m} D^{j} Y(\zeta)\right\} X_{+}(\zeta)^{q},
$$

where $m \geq 2, j \leq m$, and $p+m+q=n$.

3.3. Exponential commutator formula. We now apply Corollary 3.7 to the commutator formula (12) in Corollary 3.4. After cancellations we find that the linear and quadratic terms in $X$ are in the image of the map $\sigma: S(\mathfrak{g}) \rightarrow$ $\operatorname{Hom}(M, M\{\zeta\})$. Furthermore, all the higher-order terms in $X$ involve $(\operatorname{ad} X)^{m}$ for $m \geq 3$, as follows:

Proposition 3.8. Let $X, Y \in \mathfrak{g}$. Then

$$
\begin{aligned}
{\left[: e^{t X\left(\zeta_{1}\right)}\right.} & \left.:, Y\left(\zeta_{2}\right)\right] \\
= & (D \delta)\left(\zeta_{1} / \zeta_{2}\right)\left\{c B(t X, Y) \sigma\left(e^{t X}\right)\left(\zeta_{1}\right)+\frac{1}{2} \sigma\left(e^{t X}(\operatorname{ad} t X)^{2} Y\right)\left(\zeta_{1}\right)\right\} \\
& +\delta\left(\zeta_{1} / \zeta_{2}\right) \sigma\left(e^{t X}(\operatorname{ad} t X) Y\right)\left(\zeta_{1}\right)+R(t X, Y)\left(\zeta_{1}, \zeta_{2}\right)
\end{aligned}
$$


Here the remainder $R(t X, Y)\left(\zeta_{1}, \zeta_{2}\right)$ is a formal sum of operators homogeneous of degree $n \geq 3$ in $t$. Each of these homogeneous components is a finite linear combination of terms of the form

$$
t^{n} D^{i} \delta\left(\zeta_{1} / \zeta_{2}\right) X_{-}\left(\zeta_{1}\right)^{p}(\operatorname{ad} X)^{m} D^{j} Y\left(\zeta_{1}\right) X_{+}\left(\zeta_{1}\right)^{q}
$$

where $m \geq 3, i+j \leq m-1$, and $p+m+q=n$.

Remark. The key property of the remainder terms (2) is that they all involve $(\operatorname{ad} X)^{m}(Y)$ for $m \geq 3$.

Proof. Let us call a formal linear combination of terms of the form (2) an acceptable remainder if the exponent $m$ of ad $X$ is $\geq 3, i+j \leq m-1$, and there is only a finite number of terms with a given total degree $p+m+q$ in $X$. By Corollary 3.7 we have

$$
\begin{gathered}
(\operatorname{ad} t X) \delta\left(\zeta_{1} / \zeta_{2}\right) e^{t X_{-}\left(\zeta_{1}\right)} Y\left(\zeta_{1}\right) e^{t X_{+}\left(\zeta_{1}\right)}=\delta\left(\zeta_{1} / \zeta_{2}\right) \sigma\left(e^{t X}(\operatorname{ad} t X) Y\right)\left(\zeta_{1}\right) \\
-\frac{(\operatorname{ad} t X)^{2}}{2} \delta\left(\zeta_{1} / \zeta_{2}\right) e^{t X_{-}\left(\zeta_{1}\right)}\left\{(D+1) Y\left(\zeta_{1}\right)\right\} e^{t X_{+}\left(\zeta_{1}\right)}
\end{gathered}
$$

plus an acceptable remainder. Substituting this in the right side of formula (12) of $\S 3.1$, we obtain (1).

3.4. Commutator formula for Sugawara fields. We can now state our first main result. We assume that $\mathfrak{g}$ is simple and $B$ is the Killing form.

Theorem 3.9 (Generating function version of commutator formula). Let $X, Y$ $\in \mathfrak{g}$. Then on any module in the category $\mathscr{F}$,

$$
\begin{aligned}
{\left[\int_{G}:\right.} & \left.e^{t X^{g}\left(\zeta_{1}\right)}: d g, Y\left(\zeta_{2}\right)\right] \\
= & \left(c+\frac{1}{2}\right)(D \delta)\left(\zeta_{1} / \zeta_{2}\right) \sigma\left(\int_{G} e^{t X^{g}} B\left(t X^{g}, Y\right) d g\right)\left(\zeta_{1}\right) \\
& +\Phi(t X, Y)\left(\zeta_{1}, \zeta_{2}\right)
\end{aligned}
$$

Here the remainder $\Phi(t X, Y)\left(\zeta_{1}, \zeta_{2}\right)$ is a formal sum of operators homogeneous of degree $n \geq 3$ in $t$. The coefficient of $t^{n}$ in $\Phi(t X, Y)\left(\zeta_{1}, \zeta_{2}\right)$ is a finite linear combination of operators of the form

$$
D^{i} \delta\left(\zeta_{1} / \zeta_{2}\right) \int_{G} X_{-}^{g}\left(\zeta_{1}\right)^{p}\left(\operatorname{ad} X^{g}\right)^{m} D^{j} Y\left(\zeta_{1}\right) X_{+}^{g}\left(\zeta_{1}\right)^{q} d g
$$

where $m \geq 3, i+j \leq m-1$, and $p+m+q=n$.

Proof. We replace $X$ by $X^{g}$ in formula (1) of $\S 3.3$ and integrate over $G$. By Corollary 1.4 , the integral involving ad $X^{g}$ vanishes. We apply formula (9) of $\S 1.2$ to the integral involving $\left(\mathrm{ad} X^{g}\right)^{2}$ and combine terms to obtain (1).

Corollary 3.10. Let $Q \in S^{2}(\mathfrak{g})^{G}$ correspond to the Killing form. Then for $Y \in \mathfrak{g}$,

$$
\left[\sigma(Q)\left(\zeta_{1}\right), Y\left(\zeta_{2}\right)\right]=(2 c+1)(D \delta)\left(\zeta_{1} / \zeta_{2}\right) Y\left(\zeta_{1}\right)
$$


Proof. Taking the quadratic terms in $t$ in identity (1) and applying equation (4) of $\S 1.2$, we obtain

$$
\left[\int_{G}: X^{g}\left(\zeta_{1}\right):{ }^{2} d g, Y\left(\zeta_{2}\right)\right]=\frac{B(X, X)}{\operatorname{dim} \mathfrak{g}}(2 c+1)(D \delta)\left(\zeta_{1} / \zeta_{2}\right) Y\left(\zeta_{1}\right) .
$$

On the other hand, since $\mathfrak{g}$ is simple, we know by Schur's lemma that

$$
\int_{G}\left(X^{g}\right)^{2} d g=\alpha(X) Q
$$

for some scalar $\alpha(X)$. Applying the operator $\nabla_{Y}$ to both sides of this equation and using formula (4) of $\S 1.2$, we find that $\alpha(X)=B(X, X) / \operatorname{dim} g$. Thus (3) follows from (4).

Let $T_{n} \in \operatorname{Hom}(M, M)$ be the coefficient of $\zeta^{n}$ in $\sigma(Q)(\zeta)$. These operators are the well-known quadratic Sugawara operators (cf. [G-W3, Lemma 2.1], where the name "shifted Casimir operators" is used). If we write out the formal series (3) in $\zeta_{1}^{m} \zeta_{2}^{n}$ and equate corresponding terms on each side, we obtain the following commutation relations.

Corollary 3.11. Let $Y \in \mathfrak{g}$. Then for all $m, n \in \mathbf{Z}$

$$
\left[Y(m), T_{n}\right]=m(2 c+1) Y(m+n)
$$

on any module in the category $\mathscr{F}$.

For a general Sugawara field $\sigma(u)(\zeta)$, we have the following commutation relations with the current fields.

Corollary 3.12 (Homogeneous version of commutator formula). Let $u \in S^{n}(\mathfrak{g})^{G}$. Then for $Y \in \mathfrak{g}$,

$$
\left[\sigma(u)\left(\zeta_{1}\right), Y\left(\zeta_{2}\right)\right]=\left(c+\frac{1}{2}\right)(D \delta)\left(\zeta_{1} / \zeta_{2}\right) \sigma\left(\nabla_{Y} u\right)\left(\zeta_{1}\right)+R(u, Y)\left(\zeta_{1}, \zeta_{2}\right)
$$

on any module in the category $\mathscr{F}$. Here the remainder $R(u, Y)$ is a finite linear combination of operators of the form (2), with $X$ running over a finite subset of $\mathfrak{g}, m \geq 3, i+j \leq m-1$, and $p+m+q=n$.

Proof. Equating terms homogeneous of degree $n$ in $t$ from formula (1), we obtain formula (6) for invariants of the form

$$
u=\int_{G}\left(X^{g}\right)^{n} d g
$$

with $X \in \mathfrak{g}$. Let $\left\{X_{i}\right\}$ be a basis of $\mathfrak{g}$, and define $\Xi_{n}$ to be the set of elements $\sum k_{i} X_{i}$, with $0 \leq k_{i} \leq n$ integers. Then any polynomial of degree $n$ on $\mathfrak{g}$ that vanishes on $\Xi_{n}$ must be identically zero. Hence the finite set $\left\{X^{n}: X \in\right.$ $\left.\Xi_{n}\right\}$ spans $S^{n}(\mathfrak{g})$. It follows that invariants of the form (7), with $X \in \Xi_{n}$, $\operatorname{span} S^{n}(\mathfrak{g})^{G}$. Hence formula (6), with the remainder as stated, is true for any invariant. 


\section{Restricted Sugawara fieldS}

4.1. Invariants arising in the commutation formula. We now assume that $\mathfrak{g}$ is simple and take $B$ to be the Killing form. Let $M$ be a module in the category $\mathscr{F}$. When $M$ is an irreducible highest-weight module on which $c+\frac{1}{2} \neq 0$, the quadratic Sugawara operators $\left\{T_{n}\right\}$ in Corollary 3.11 can be normalized to furnish a representation of the Virasoro algebra on $M$ [G-W3, Lemma 2.2]. On the other hand, when $c+\frac{1}{2}=0$ on $M$ then the operators $T_{n}$ commute with the action of $\hat{\mathfrak{g}}$ on the module. We now study this phenomenon in general.

Definition. Let $u \in S(\mathfrak{g})^{G}$. Then $\sigma(u)(\zeta)$ is a restricted Sugawara field if on all modules in the category. $\mathscr{F}$

$$
\left[\sigma(u)\left(\zeta_{1}\right), Y\left(\zeta_{2}\right)\right]=\left(c+\frac{1}{2}\right)(D \delta)\left(\zeta_{1} / \zeta_{2}\right) \sigma\left(\nabla_{Y} u\right)\left(\zeta_{1}\right)
$$

for all $Y \in \mathfrak{g}$. (Here $\zeta_{1}$ and $\zeta_{2}$ are formal variables.)

Suppose $\sigma(u)(\zeta)$ is a restricted Sugawara field and $M$ is a module in the category $\mathscr{F}$ on which $c+\left(\frac{1}{2}\right)=0$. Then the $\zeta$-homogeneous components of $\sigma(u)(\zeta)$ commute with the action of $\hat{\mathfrak{g}}$ on $M$, by Lemma 2.1 , and will be called Sugawara operators.

The quadratic invariant $Q$ associated with the Killing form defines a restricted Sugawara field, by Corollary 3.10. For a general invariant $u$, the operator $\sigma(u)(\zeta)$ is a restricted Sugawara field if and only if the remainder term $R(u, Y)\left(\zeta_{1}, \zeta_{2}\right)$ described in Corollary 3.12 vanishes on all modules $M$ in the category $\mathscr{F}$. We shall analyze this remainder using information about the $G$-invariants in the tensor algebra of $\mathfrak{g}$. For this, we introduce the following maps.

Given $X, Y \in \mathfrak{g}$ and nonnegative integers $p, m, q$, we define

$$
T_{p m q}(X, Y)=\int_{G}\left(X^{g}\right)^{\otimes p} \otimes\left(\operatorname{ad} X^{g}\right)^{m} Y \otimes\left(X^{g}\right)^{\otimes q} d g .
$$

Observe that for $X \in \mathfrak{g}$ fixed, $Y \rightarrow T_{p m q}(X, Y)$ is in $\operatorname{Hom}_{G}\left(\mathfrak{g}, \otimes^{p+q+1} \mathfrak{g}\right)$. We define a linear map

$$
\Phi_{p m q}: \otimes^{p+q+1} \mathfrak{g} \rightarrow \operatorname{Hom}(M, M\{\zeta\})
$$

by setting

$$
\begin{aligned}
\Phi_{p m q}\left(X^{1} \otimes \cdots\right. & \left.\otimes X^{p} \otimes Y \otimes X^{p+1} \otimes \cdots \otimes X^{p+q}\right)(\zeta) \\
& =X_{-}^{1}(\zeta) \cdots X_{-}^{p}(\zeta)\left\{D^{m} Y(\zeta)\right\} X_{+}^{p+1}(\zeta) \cdots X_{+}^{p+q}(\zeta)
\end{aligned}
$$

for elements $X^{i}, Y \in \mathfrak{g}$.

Suppose $u \in S(\mathfrak{g})^{G}$ is homogeneous of degree $n$. Then the remainder term $R(u, Y)\left(\zeta_{1}, \zeta_{2}\right)$ in the commutation formula in Corollary 3.12 can be written as a finite linear combination of terms of the form

$$
D^{i} \delta\left(\zeta_{1} / \zeta_{2}\right) \Phi_{p j q}\left(T_{p m q}(X, Y)\right)\left(\zeta_{1}\right)
$$

with $X \in \mathfrak{g}$ and the integers $p, m, q$ satisfying $m \geq 3$ and $p+m+q=n$. So the possible remainder terms are restricted by the possible maps $T_{p m q}(X, Y)$. 
Theorem 4.1. (a) Let $u \in S^{3}(\mathfrak{g})^{G}$. Then $\sigma(u)(\zeta)$ is a restricted Sugawara field.

(b) Let $\omega: S(\mathfrak{g}) \rightarrow U(\mathfrak{g})$ be the canonical symmetrization map, and let $\chi: U(\mathfrak{g})^{G} \rightarrow \mathbf{C}$ be the infinitesimal character of the adjoint representation on $\mathfrak{g}$. If $u \in S^{4}(\mathfrak{g})^{G}$ satisfies

$$
\chi(\omega(u))=0
$$

then $\sigma(u)(\zeta)$ is a restricted Sugawara field.

Proof. For invariants of degree 3, all the remainder terms of the form (4) involve the map $T_{0,3,0}(X, Y)$. But this map vanishes by Proposition 1.7. Hence part (a) of the theorem is proved.

To prove part (b), we first prove that

$$
T_{p m q}(X, Y)=\gamma(X) T_{p m q}(Y)
$$

when $p+m+q=4$ and $m \geq 3$, where $\gamma(X)=\operatorname{tr}_{\mathfrak{g}}\left((\operatorname{ad} X)^{4}\right) / \operatorname{dim} \mathfrak{g}$ and $T_{p m q}(Y)$ does not depend on $X$. There are two cases to consider.

Case 1. $m=4$ and $p=q=0$. Since $\mathfrak{g}$ is simple, Schur's lemma immediately implies that $T_{0,4,0}(X, Y)=\gamma(X) Y$.

Case 2. $m=3$. We have

$$
T_{1,3,0}(X, Y)+T_{0,3,1}(X, Y)=\int_{G}\left(\operatorname{ad} X^{g}\right)^{3}\left\{X^{g} \otimes Y+Y \otimes X^{g}\right\} d g .
$$

This vanishes by Proposition 1.7, however, since the integrand is a symmetric tensor. On the other hand,

$$
T_{1,3,0}(X, Y)-T_{0,3,1}(X, Y)=2 \int_{G} X^{g} \wedge\left(\operatorname{ad} X^{g}\right)^{3} Y d g
$$

where $\wedge$ denotes the product in the exterior algebra of $\mathfrak{g}$. Hence the map

$$
Y \rightarrow T_{1,3,0}(X, Y)-T_{0,3,1}(X, Y)
$$

is in $\operatorname{Hom}_{G}\left(\mathfrak{g}, \Lambda^{2} \mathfrak{g}\right)$. We now use the following general fact:

Lemma 4.2. Let $\left\{Z_{i}\right\}$ be an orthonormal basis for $\mathfrak{g}$ relative to the form $B$. Then $\operatorname{dim} \operatorname{Hom}_{G}\left(\mathfrak{g}, \bigwedge^{2} \mathfrak{g}\right)=1$ with basis the map

$$
J(Y)=\sum_{i<j} B\left(Y,\left[Z_{i}, Z_{j}\right]\right) Z_{i} \wedge Z_{j} .
$$

Proof. See §A.2.

We can now finish the proof of (6) in Case 2. By the remarks above it suffices to consider the map (7), which we can write as $\beta(X) J(Y)$ for some $\beta(X) \in \mathbf{C}$ by Lemma 4.2. Hence we only need to show that $\beta(X)$ is proportional to $\operatorname{tr}_{\mathfrak{g}}\left((\operatorname{ad} X)^{4}\right)$. We observe that

$$
\left(J(Y), Z_{i} \wedge Z_{j}\right)=\frac{1}{2} B\left(Y,\left[Z_{i}, Z_{j}\right]\right)
$$


for all $Y \in \mathfrak{g}$. Taking the inner product of the right side of (7) with $Z_{i} \wedge Z_{j}$ and comparing terms with (8), we calculate that

(9) $\beta(X)\left[Z_{i}, Z_{j}\right]=2 \int_{G}\left\{B\left(X^{g}, Z_{j}\right)\left(\operatorname{ad} X^{g}\right)^{3} Z_{i}-B\left(X^{g}, Z_{i}\right)\left(\operatorname{ad} X^{g}\right)^{3} Z_{j}\right\} d g$.

Now we take the inner product of both sides of $(9)$ with $\left[Z_{i}, Z_{j}\right]$ and sum over $i$, $j$. Since $\sum\left(\operatorname{ad} Z_{i}\right)^{2}=1$, we obtain the formula

$$
\begin{aligned}
\beta(X) \operatorname{dim} \mathfrak{g} & =4 \sum_{i, j} \int_{G} B\left(X^{g}, Z_{j}\right) B\left(\left(\operatorname{ad} X^{g}\right)^{3} Z_{i},\left[Z_{i}, Z_{j}\right]\right) d g \\
& =4 \sum_{i} \int_{G} B\left(\left(\operatorname{ad} X^{g}\right)^{4} Z_{i}, Z_{i}\right) d g \\
& =4 \operatorname{tr}_{\mathfrak{g}}\left((\operatorname{ad} X)^{4}\right) .
\end{aligned}
$$

This completes the proof of (6).

We now complete the proof of part (b) of the theorem. Since the symmetrization map $\omega$ satisfies $\omega\left(X^{k}\right)=\omega(X)^{k}$ for $X \in \mathfrak{g}$ and intertwines the adjoint action of $G$ on $S(\mathfrak{g})$ and $U(\mathfrak{g})$, we can write

$$
\gamma(X)=\chi\left(\omega\left(I\left(X^{4}\right)\right)\right)
$$

where $I\left(X^{n}\right)$ is the projection of $X^{n}$ onto the invariants, given by formula (2) of $\S 1.1$. Thus by using (6) in (4), we can write the remainder term as

$$
R(u, Y)\left(\zeta_{1}, \zeta_{2}\right)=\chi(\omega(u)) T(Y)\left(\zeta_{1}, \zeta_{2}\right) \text {. }
$$

Here $T(Y)\left(\zeta_{1}, \zeta_{2}\right)$ is a finite sum of operators of the form

$$
\left(D^{i} \delta\right)\left(\zeta_{1} / \zeta_{2}\right) T_{i}(Y)\left(\zeta_{1}\right)
$$

with $T_{i}(Y)(\zeta) \in \operatorname{Hom}_{\infty}(M, M\{\zeta\})$ and depending linearly on $Y$. In particular, if $u$ satisfies (5), then the remainder vanishes, and hence $\sigma(u)(\zeta)$ is a restricted Sugawara field.

Remark. We have calculated an explicit formula for the operator $T(Y)\left(\zeta_{1}, \zeta_{2}\right)$ in (10), but it would serve no purpose to give it here. The important point is that $T(Y)\left(\zeta_{1}, \zeta_{2}\right)$ does not depend on $u$.

Corollary 4.3. Let $Q \in S^{2}(\mathfrak{g})^{G}$ correspond to the Killing form. Given $u \in$ $S^{4}(\mathfrak{g})^{G}$, set

$$
u^{\#}=u-\chi(\omega(u)) Q^{2} .
$$

Then $\sigma\left(u^{\#}\right)(\zeta)$ is a restricted Sugawara field.

Proof. If $\pi$ is any irreducible finite-dimensional representation of $\mathfrak{g}$, then

$$
\pi\left(\omega\left(Q^{2}\right)\right)=\pi(\omega(Q))^{2}
$$

as we verify by writing $Q$ symmetrically in terms of a root basis and evaluating the operators on the highest-weight vector. Taking $\pi$ to be the adjoint representation, in which the Casimir operator $\omega(Q)$ acts by 1 , we conclude that

$$
\chi\left(\omega\left(Q^{2}\right)\right)=1 \text {. }
$$


It follows that $\chi\left(u^{\#}\right)=0$, so we may apply part (b) of Theorem 4.1.

We now consider the invariants of the special form

$$
u_{k}=\int_{G}\left(X^{g}\right)^{k} d g
$$

for $X \in \mathfrak{g}$. We shall show that $\sigma\left(u_{k}\right)(\zeta)$ is a restricted Sugawara field if $X$ lies in the zero variety of certain $G$-invariant polynomials on $\mathfrak{g}$. To describe this variety, we need some more notation.

Let $\left\{Z_{i}\right\}$ be an orthonormal basis for $\mathfrak{g}$ relative to the form $B$. For $X \in \mathfrak{g}$ and $m, r$ nonnegative integers, define

$$
S_{m r}(X)=\sum_{i=1}^{\operatorname{dim} g} \int_{G} Z_{i} \otimes\left(\operatorname{ad} X^{g}\right)^{m} Z_{i} \otimes\left(X^{g}\right)^{\otimes r} d g .
$$

Let $k \geq 3$ be an integer and define $\mathscr{R}^{k}$ to be the linear span of the polynomials

$$
X \rightarrow\left\langle S_{m r}(X), J\right\rangle, \text { for } m \geq 3, m+r=k,
$$

as $J$ ranges over the $G$-invariants in $\otimes^{r+2} \mathfrak{g}^{*}$. It is clear that $\mathscr{R}^{k} \subset S^{k}\left(\mathfrak{g}^{*}\right)^{G}$.

Example. We have $\mathscr{R}^{3}=0$ and $\mathscr{R}^{4}$ consisting of multiples of the polynomial $\operatorname{tr}_{\mathfrak{g}}\left((\operatorname{ad} X)^{4}\right)$. (This follows from the proofs of Theorems 4.1 and 4.4.)

Theorem 4.4. Let $X \in \mathfrak{g}$ and define $u_{k}$ in terms of $X$ by equation (11). Suppose $X$ satisfies the equations

$$
f(X)=0 \text { for all } f \in \mathscr{R}^{k} .
$$

Then $\sigma\left(u_{k}\right)(\zeta)$ is a restricted Sugawara field.

Proof. By Corollary 3.10 and part (a) of Theorem 4.1, we only need to consider the case $k \geq 4$. From Corollary 3.12 and equation (4), we have the following sufficient condition for the restricted Sugawara commutation property:

(a) Suppose $T_{p m q}(X, Y)=0$ for all $Y \in \mathfrak{g}$ and all integers $m \geq 3$, $p \geq 0$, and $q \geq 0$ with $p+m+q=k$. Then $\sigma\left(u_{k}\right)(\zeta)$ is a restricted Sugawara field.

We next express the vanishing of $T_{p m q}(X, Y)$ for all $Y \in \mathfrak{g}$ in terms of orthogonality relations in the tensor algebra of $\mathfrak{g}$. Extend the form $B$ to a nondegenerate bilinear form $(\cdot, \cdot)$ on $\otimes^{n} \mathfrak{g}$ and define $S_{p m q}(X) \in \otimes^{p+q+2} \mathfrak{g}$ as follows: Given $W_{i}, Y, Z$ in $\mathfrak{g}$, let

$$
\begin{aligned}
\left(S_{p m q}(X), Y \otimes W_{1} \otimes \cdots \otimes W_{p} \otimes Z \otimes W_{p+1} \otimes \cdots \otimes W_{p+q}\right) & \\
& =\left(T_{p m q}(X, Y), W_{1} \otimes \cdots \otimes W_{p} \otimes Z \otimes W_{p+1} \otimes \cdots \otimes W_{p+q}\right) .
\end{aligned}
$$

From equation (2) we see that

$$
\begin{aligned}
\left(S_{p m q}(X), Y \otimes W_{1} \otimes \cdots \otimes W_{p} \otimes Z \otimes W_{p+1} \otimes \cdots \otimes W_{p+q}\right) & \\
& =\int_{G} B\left(\left(\operatorname{ad} X^{g}\right)^{m} Y, Z\right)\left\{\prod_{j=1}^{p+q} B\left(X^{g}, W_{j}\right)\right\} d g .
\end{aligned}
$$


Fix positive integers $k \geq m \geq 3$ and set $r=k-m$. Let $X \in \mathfrak{g}$. We claim that the following conditions (b) and (c) are equivalent: $T_{p m q}(X, Y)=0$ for all $Y \in \mathfrak{g}$ and all nonnegative integers $p, q$
with $p+q=r$. (c) For every $G$-invariant tensor $J \in \otimes^{r+2} \mathfrak{g}^{*}$ one has $\left\langle S_{0 m r}(X), J\right\rangle$
$=0$.

To prove this equivalence, we observe that condition (b) is the same as the vanishing of $S_{p m q}(X)$ for all $p, q$ with $p+q=r$. But by formula (14), $S_{p m q}(X)=0$ for all $p, q$ with $p+q=r$ if and only if $S_{0 m r}(X)=0$. In particular, condition (b) implies condition (c). For the converse, suppose (c) holds. We observe from (14) that $S_{0 m r}(X)$ is invariant under the action of $G$ on $\bigotimes^{r+2} \mathfrak{g}$. Since the $G$-invariants in $\bigotimes^{r+2} \mathfrak{g}^{*}$ are nonsingularly paired with the $G$-invariants in $\bigotimes^{r+2} \mathfrak{g}$, it follows that $S_{0 m r}(X)=0$. Hence (b) holds.

Let $\left\{Z_{i}\right\}$ be an orthonormal basis for $\mathfrak{g}$ relative to the form $B$. Then $\left\{Z_{i_{1}} \otimes \cdots \otimes Z_{i_{p}}\right\}$ is an orthonormal basis for $\otimes^{p} \mathfrak{g}$. Using equation (14) to expand $S_{0 m r}(X)$ relative to this basis, we find that $S_{m r}(X)=S_{0 m r}(X)$. The theorem now follows from (a) and the equivalence of (b) and (c).

4.2. Commutator invariants for $\mathrm{SU}(n)$. Throughout this section we take $\mathfrak{g}=$ $\mathrm{sl}_{n}(\mathrm{C})$ and $G=\mathrm{SU}(n)$. We shall use classical invariant theory to determine an explicit basis for the subspace $\mathscr{R}^{k}$ of $G$-invariant polynomials on $\mathfrak{g}$ that occurs in Theorem 4.4 .

Let $M_{n}(\mathbf{C})$ be the associative algebra of $n \times n$ complex matrices. Let $\Sigma_{p}$ denote the symmetric group on $p$ letters. Given $\sigma \in \Sigma_{p}$, let $\gamma_{1}, \gamma_{2}, \ldots, \gamma_{q}$ be the disjoint cycles of $\sigma$ and define $J_{\sigma} \in \bigotimes^{P} M_{n}(\mathbf{C})^{*}$ by

$$
\left\langle J_{\sigma}, Y_{1} \otimes \cdots \otimes Y_{p}\right\rangle=\prod_{i=1}^{q} \operatorname{tr}_{\mathbf{C}^{n}}\left(Y_{\left[\gamma_{i}\right]}\right) .
$$

Here we set $Y_{\left[\gamma_{i}\right]}=Y_{j_{1}} Y_{j_{2}} \cdots Y_{j_{s}}$ if $\gamma_{i}$ is the cycle $\left(j_{1} j_{2} \cdots j_{s}\right)$, the product being taken in $M_{n}(\mathbf{C})$. We let $g \in \mathrm{GL}_{n}(\mathbf{C})$ act on $\Theta^{p} M_{n}(\mathbf{C})$ by

$$
\left(Y_{1} \otimes \cdots \otimes Y_{p}\right)^{g}=g^{-1} Y_{1} g \otimes \cdots \otimes g^{-1} Y_{p} g \text {. }
$$

It is obvious that $J_{\sigma}$ is invariant under the transpose of this action.

Lemma 4.5. The space of $G$-invariants in $\bigotimes^{p} \mathfrak{g}^{*}$ is spanned by the restrictions to $\bigotimes^{p} \mathfrak{g}$ of the elements $\left\{J_{\sigma}: \sigma \in \Sigma_{p}\right\}$.

Proof. Let $\pi: M_{n}(\mathbf{C}) \rightarrow \mathfrak{g}$ be the projection

$$
\pi(Y)=Y-\operatorname{tr}_{\mathbf{C}^{n}}(Y) I .
$$

Then $\pi(Y)^{g}=\pi\left(Y^{g}\right)$. Hence if $J$ is a $G$-invariant in $\otimes^{p} \mathfrak{g}^{*}$, then $\left(\pi^{*}\right)^{\otimes p} J$ is a $\mathrm{GL}_{n}(\mathbf{C})$-invariant in $\bigotimes^{p} M_{n}(\mathbf{C})^{*}$. Thus the restriction map on invariants is surjective. So it suffices to know that the space of $\mathrm{GL}_{n}(\mathbf{C})$-invariants 
in $\otimes^{p} M_{n}(\mathbf{C})^{*}$ is spanned by $\left\{J_{\sigma}: \sigma \in \Sigma_{p}\right\}$. This "fundamental theorem of invariant theory" is a consequence of the following properties:

(a) Let $J \in \bigotimes^{p} M_{n}(\mathbf{C})=\operatorname{End}\left(\otimes^{p} \mathbf{C}^{n}\right)$. Then $J$ is invariant under $\mathrm{GL}_{n}(\mathbf{C})$ if and only if the action of $J$ on $\otimes^{p} \mathrm{C}^{n}$ commutes with the action of $\mathrm{GL}_{n}(\mathrm{C})$.

(b) The commutant of the representation of $\mathrm{GL}_{n}(\mathbf{C})$ on $\otimes^{p} \mathrm{C}^{n}$ is spanned by the permutation operators

$$
\pi_{\sigma}\left(v_{1} \otimes \cdots \otimes v_{p}\right)=v_{\sigma 1} \otimes \cdots \otimes v_{\sigma p}
$$

for $\sigma$ ranging over $\Sigma_{p}$ [Wey, Chapter 4].

To complete the proof, take $\pi_{\sigma}$ in property (b) and let $J_{\sigma}$ be the element of $\otimes^{p} M_{n}(\mathbf{C})^{*}$ defined by $\pi_{\sigma}$ via the trace form. Then one calculates that $J_{\sigma}$ is given by formula (1) (cf. [Mur, equation 3.22]).

Lemma 4.6. Let $X \in \mathfrak{g}$ and let $m \geq 3, r \geq 0$, with $m+r \geq 4$. Suppose $\sigma \in \Sigma_{r+2}$. One of the following occurs:

Case 1. Suppose 1 and 2 are in disjoint cycles of $\sigma$. Then

$$
\left\langle J_{\sigma}, S_{m r}(X)\right\rangle=0 \text {. }
$$

Case 2. Suppose 1 and 2 are in the same cycle of $\sigma$, and suppose $\sigma$ has cycle decomposition $\gamma_{1} \cdots \gamma_{q}$, enumerated so that 1 and 2 are in the cycle $\gamma_{1}$. Let $k_{i}$ be the length of the cycle $\gamma_{i}$. Then

$$
\left\langle J_{\sigma}, S_{m r}(X)\right\rangle=\operatorname{tr}_{M_{n}(\mathbf{C})}\left(L(X)^{a}(\operatorname{ad} X)^{m} R(X)^{b}\right) \prod_{i=2}^{q} \operatorname{tr}_{\mathbf{C}^{n}}\left(X^{k_{i}}\right)
$$

where $a, b$ are nonnegative integers with $a+b+2=k_{1}$. Here $L(X)$ (resp. $R(X)$ ) denotes the action of left (resp. right) multiplication by $X$ on $M_{n}(\mathbf{C})$.

Proof. It is more convenient to use the invariant form

$$
B(X, Y)=\operatorname{tr}_{\mathbf{C}^{n}}(X Y)
$$

which is proportional to the Killing form. This has the effect of multiplying $S_{m r}(X)$ by a nonzero constant. Since we are only interesting in the vanishing of $S_{m r}(X)$, we shall abuse notation and use formula (12) of $\S 4.1$ with a $B$ orthonormal basis $Z_{1}, \ldots, Z_{d}$ for $\mathfrak{g}\left(d=n^{2}-1\right)$. We set $Z_{0}=n^{-1 / 2} I_{n}$. Then $\left\{Z_{i}: i=0, \ldots, d\right\}$ is an orthonormal basis for $M_{n}(\mathbf{C})$ relative to $B$. Since $(\operatorname{ad} X) Z_{0}=0$ for $X \in \mathfrak{g}$, the summation in formula (12) of $\S 4.1$ can include $i=0$ provided $m \geq 1$. This will allow us to use the associative algebra structure on $M_{n}(\mathbf{C})$ in our calculations involving $S_{m r}(X)$. Set

$$
T(X)=\sum_{i=0}^{d} Z_{i} \otimes(\operatorname{ad} X)^{m} Z_{i} \otimes(X)^{\otimes r}
$$

Then $S_{m r}(X)$ is obtained by integrating the function $g \rightarrow T\left(X^{g}\right)$ over $G$, by the remarks just made. 
Suppose now we are in the situation of Case 1. Then by (1) we have

$$
\left\langle J_{\sigma}, T(X)\right\rangle=\sum_{i=0}^{d} \operatorname{tr}_{\mathbf{C}^{n}}\left(Z_{i} X^{a}\right) \operatorname{tr}_{\mathbf{C}^{n}}\left(X^{b}(\operatorname{ad} X)^{m} Z_{i}\right) P(X),
$$

where $a$ and $b$ are nonnegative integers and $P(X)$ is a product of factors of the form $\operatorname{tr}_{C^{n}}\left(X^{k}\right)$. We observe that the sum over $i$ in (4) gives the expansion of the element $X^{a}$ in $M_{n}(\mathbf{C})$. Hence

$$
\left\langle J_{\sigma}, T(X)\right\rangle=\operatorname{tr}_{\mathbf{C}^{n}}\left(X^{b}(\operatorname{ad} X)^{m} X^{a}\right) P(X)=0 .
$$

Since $X \in \mathfrak{g}$ was arbitrary, this same equation holds with $X$ replaced by $X^{g}$, for $g \in G$. Integrating over $G$, we obtain (2).

Now suppose we are in the situation of Case 2. Write the cycle $\gamma_{1}=$ $\left(1 i_{1} \cdots i_{a} 2 j_{1} \cdots j_{b}\right)$. Then by formula (1) we have

$$
\left\langle J_{\sigma}, T(X)\right\rangle=\sum_{i=0}^{d} \operatorname{tr}_{\mathbf{C}^{n}}\left(Z_{i} X^{a}(\operatorname{ad} X)^{m} Z_{i} X^{b}\right) \prod_{j=2}^{q} \operatorname{tr}_{\mathbf{C}^{n}}\left(X^{k_{j}}\right) .
$$

But the sum over $i$ gives the trace of the operator $L(X)^{a}(\operatorname{ad} X)^{m} R(X)^{b}$ on $M_{n}(\mathbf{C})$. Since $X \in \mathfrak{g}$ was arbitrary, this same equation holds with $X$ replaced by $X^{g}$, for $g \in G$. Integrating over $G$ and using the $G$-invariance of the traces, we obtain (3).

Define $G$-invariant polynomials $s_{k}$ on $\mathfrak{g}$ by

$$
s_{k}(X)=\operatorname{tr}_{\mathbf{C}^{n}}\left(X^{k}\right)
$$

for $k=0,1,2, \ldots$. One has $s_{0}=n$ and $s_{1}=0$. The polynomials $s_{2}, \ldots, s_{n}$ are algebraically independent generators for the ring of $G$-invariant polynomials on $\mathfrak{g}$. We denote by $\mathscr{P}$ the set of all sequences $p=\left(p_{1}, p_{2}, \ldots\right)$ of nonnegative integers such that $p_{i}=0$ for all sufficiently large $i$. Given $p \in \mathscr{P}$, we set

$$
s^{p}=\prod_{i=1}^{\infty} s_{i}^{p_{i}}
$$

Since all but a finite number of factors in the product are $1, s^{p}$ is a polynomial on $\mathfrak{g}$ of degree

$$
w(p)=\sum_{i=1}^{n} i p_{i}
$$

Since $s_{1}=0$, we see that if $p_{1} \neq 0$ then $s^{p}=0$.

We define

$$
\begin{aligned}
& \mathscr{P}_{k}=\{p \in \mathscr{P}: w(p)=k\}, \\
& \mathscr{P}_{n, k}=\left\{p \in \mathscr{P}_{k}: p_{i}=0 \text { for } i=1 \text { and } i>n\right\} .
\end{aligned}
$$

The polynomials

$$
\left\{s^{p}: p \in \mathscr{P}_{n, k}\right\}
$$


are then a basis for the $G$-invariant polynomials of degree $k$ on $\mathfrak{g}$, for $k \geq 2$.

We note for use in $\S 4.3$ that each $p \in \mathscr{P}_{k}$ corresponds to a partition

$$
k=m_{1}+m_{2}+\cdots
$$

with $m_{j}=i$ for $p_{i}$ values of $j$. (This partition is also denoted by $1^{p_{1}} 2^{p_{2}} \ldots$.) One has

$$
s^{p}=s_{m_{1}} s_{m_{2}} \cdots .
$$

For integers $u \geq 4$ and $0 \leq q \leq u-3$, define

$$
\beta_{u q}=s_{u-q} s_{q}-3 s_{u-q-1} s_{q+1}+3 s_{u-q-2} s_{q+2}-s_{u-q-3} s_{q+3} .
$$

Because $s_{1}=0$, we have

$$
\begin{aligned}
& \beta_{u 0}=s_{u} s_{0}+3 s_{u-2} s_{2}-s_{u-3} s_{3}, \\
& \beta_{u 1}=-3 s_{u-2} s_{2}+3 s_{u-3} s_{3}-s_{u-4} s_{4} .
\end{aligned}
$$

Note that $\beta_{u q}$ is a homogeneous polynomial of degree $u$ on $\mathfrak{g}$. Since

$$
\beta_{u q}=-\beta_{u, u-q-3}
$$

we may restrict $q$ to the range $0 \leq q \leq(u-3) / 2$.

Lemma 4.7. Let $k \geq 4$. Then the space $\mathscr{R}^{k}$ is spanned by the polynomials

$$
s^{p} \beta_{u q}
$$

where $u \geq 4,0 \leq q \leq(u-3) / 2, p \in \mathscr{P}_{n, r}$, and $r+u=k$.

Proof. Define

$$
\alpha_{a b m}(X)=\operatorname{tr}_{M_{n}(\mathbf{C})}\left(L(X)^{a}(\operatorname{ad} X)^{m} R(X)^{b}\right)
$$

for $X \in \mathfrak{g}$. Then $\alpha_{a b m}$ is an invariant polynomial of degree $a+b+m$ on $\mathfrak{g}$. By Lemma $4.6 \mathscr{R}^{k}$ is spanned by the polynomials of the form

$$
\alpha_{a b m} s_{m_{1}} \cdots s_{m_{j}}
$$

that are homogeneous of degree $k$, where $m \geq 3$. Since

$$
(\operatorname{ad} X)^{m}=(L(X)-R(X))(\operatorname{ad} X)^{m-1}
$$

we have the recursion

$$
\alpha_{a b m}=\alpha_{a+1, b, m-1}-\alpha_{a, b+1, m-1} .
$$

Thus it suffices to take $m=3$ in (6). Futhermore, since

$$
\alpha_{0,0,3}(X)=\operatorname{tr}_{M_{n}(\mathbf{C})}\left((\operatorname{ad} X)^{3}\right)=0
$$

by equation (6) of $\S 1.2$, it suffices to take $a+b \geq 1$ in (6).

Finally, we can express $\alpha_{a, b, 3}$ in terms of $\beta_{u q}$ by using the relation

$$
\operatorname{tr}_{M_{n}(\mathbf{C})}\left(L(X)^{a} R(X)^{b}\right)=\operatorname{tr}_{\mathbf{C}^{n}}\left(X^{a}\right) \operatorname{tr}_{\mathbf{C}^{n}}\left(X^{b}\right) .
$$


Expanding $(\operatorname{ad} X)^{3}=(L(X)-R(X))^{3}$ and using (7), we find that

$$
\alpha_{a, b, 3}=\beta_{u q}
$$

with $u=3+a+b$ and $q=b$. Since we may assume $a+b \geq 1$, we have $u \geq 4$.

Let $u \geq 4$ be an integer. Define

$$
f_{u q}=(q-1)(u-q-1) s_{0} s_{u}+(u-1) s_{q} s_{u-q} .
$$

Note that $f_{u, 0}=f_{u, 1}=0$ and $f_{u q}=f_{u, u-q}$, so we may restrict $q$ to the range $2 \leq q \leq u / 2$. We have the following "normal basis" for $\mathscr{R}^{k}$ :

Theorem 4.8. Let $\mathfrak{g}=\operatorname{sl}_{n}(\mathbf{C})$, with $n \geq 4$. For $4 \leq k \leq n$ the space $\mathscr{R}^{k}$ has basis

$$
\left\{s^{p} f_{u q}: 4 \leq u \leq k, 2 \leq q \leq u / 2 \text {, and } p \in \mathscr{P}_{n, k-u}\right\} .
$$

Proof. Let $4 \leq u \leq n$. Define

$$
V_{u}=\operatorname{span}\left\{f_{u q}: 2 \leq q \leq u / 2\right\} .
$$

Because the polynomials $\left\{s_{k}: 2 \leq k \leq n\right\}$ are algebraically independent, it is clear from the formula for $f_{u q}$ that the set $\left\{f_{u q}: 2 \leq q \leq u / 2\right\}$ is linearly independent. Hence $\operatorname{dim} V_{u}=\operatorname{Int}(u / 2)-1$, where $\operatorname{Int}(x)$ denotes the integer part of a real number $x$. Define

$$
W_{u}=\operatorname{span}\left\{\beta_{u q}: 0 \leq q \leq(u-3) / 2\right\} .
$$

Since

$$
(u-1) \beta_{u q}=f_{u q}-3 f_{u, q+1}+3 f_{u, q+2}-f_{u, q+3}
$$

for $0 \leq q \leq(u-3) / 2$, we have $W_{u} \subset V_{u}$.

We next prove that the set

$$
\left\{\beta_{u q}: 0 \leq q \leq(u-3) / 2\right\}
$$

is linearly independent. We first observe that the variable $s_{u}$ appears only in $\beta_{u 0}$. Hence in any linear relation among the polynomials in (10), the coefficient of $\beta_{u 0}$ must be zero. Since the set (10) has $\operatorname{Int}(u / 2)-1$ elements, it thus suffices to prove that

$$
E=\operatorname{span}\left\{\beta_{u q}: 1 \leq q \leq(u-3) / 2\right\}
$$

has dimension $\operatorname{Int}(u / 2)-2$. Set $\zeta_{q}=s_{q} s_{u-q}$ and $m=\operatorname{Int}(u / 2)$. Then the subspace $E$ is contained in

$$
F=\operatorname{span}\left\{\zeta_{q}: 2 \leq q \leq m\right\} .
$$

Since $\operatorname{dim} F=m-1$, it will suffice to prove the following:

$$
\left\{\beta_{u q}: 1 \leq q \leq m-2\right\} \text { is linearly independent modulo } \mathbf{C} \zeta_{m}
$$


To prove assertion (I), suppose we have a relation

$$
\sum_{q=1}^{m-2} c_{q} \beta_{u q}=0 \quad \bmod \mathbf{C} \zeta_{m} .
$$

Expressing $\beta_{u q}$ in terms of $\left\{\zeta_{r}\right\}$ in equation (11) and comparing coefficients of $\zeta_{r}$, we find that the coefficients $c_{q}$ satisfy the system of $m-1$ equations

$$
\begin{aligned}
0 & =-3 c_{1}+c_{2}, \\
0 & =3 c_{1}-3 c_{2}+c_{3}, \\
0 & =-c_{1}+3 c_{2}-3 c_{3}+c_{4}, \\
\vdots & \\
0 & =-c_{m-5}+3 c_{m-4}-3 c_{m-3}+c_{m-2}, \\
0 & =-c_{m-4}+3 c_{m-3}-4 c_{m-2} .
\end{aligned}
$$

Here the coefficient -4 appears in the last equation because of the relation $\zeta_{m+1}=\zeta_{m}$. The first $m-3$ equations imply that

$$
c_{q}=\frac{1}{2} q(q+1) c_{1}, \text { for } 2 \leq q \leq m-2 .
$$

Substituting this formula in the last equation, we find that

$$
-c_{1}(m-1)^{2}=0 \text {. }
$$

Since $m \geq 2$, this forces $c_{1}=0$. Hence $c_{q}=0$ for all $q$, proving (I).

From (I) it follows that $\operatorname{dim} W_{u}=\operatorname{Int}(u / 2)-1=\operatorname{dim} V_{u}$. Hence $W_{u}=V_{u}$. From this and Lemma 4.7 we conclude that the set (9) is a basis for $\mathscr{R}^{k}$.

Corollary 4.9. Suppose $X \in \mathfrak{g}$ lies on the variety defined by the equations

$$
\begin{gathered}
\left(s_{3} / 2 s_{0}\right)^{2}=\left(-s_{2} / s_{0}\right)^{3}, \\
s_{2 j} / s_{0}=-(2 j-1)\left(-s_{2} / s_{0}\right)^{j}, \\
s_{2 j+1} / s_{0}=2 j\left(s_{3} / 2 s_{0}\right)\left(-s_{2} / s_{0}\right)^{j-1}
\end{gathered}
$$

for $2 \leq j \leq(n-1) / 2$, where $s_{0}=n$. Then for $4 \leq k \leq n$ one has $f(X)=0$ for all $f \in \mathscr{R}^{k}$. Furthermore, if $X$ is a point on this variety with

$$
s_{2}(X) / s_{0}=s_{3}(X) / 2 s_{0}=-1 \text {, }
$$

then for all $p \in \mathscr{P}_{k}$ one has

$$
\prod_{j \geq 2} s_{j}(X)^{p_{j}}=(-n)^{|p|} \prod_{j \geq 2}(j-1)^{p_{j}} .
$$

Proof. If $X$ satisfies equations (12)-(14), then a straightforward calculation shows that $f_{u q}(X)=0$ for $4 \leq u \leq n$ and $0 \leq q \leq u$. Hence $f(X)=0$ for all $f \in \mathscr{R}^{k}$ by Theorem 4.8. If $X$ also satisfies (15), then by (13) and (14) we have

$$
s_{j}(X)=-n(j-1)
$$

for $2 \leq j \leq n$. Hence (16) holds. 


\subsection{Restricted Sugawara fields and basic invariants.}

Definition. The affine algebra $\hat{\mathfrak{g}}$ has a complete set of restricted Sugawara fields if there exists algebraically independent homogeneous generators $u_{1}, \ldots, u_{l}$ for $S(\mathfrak{g})^{G}$, such that $\sigma\left(u_{i}\right)(\zeta)$ is a restricted Sugawara field for $i=1, \ldots, l$ (where $l=\operatorname{rank}(\mathfrak{g}))$.

Theorem 4.10. Let $\mathfrak{g}$ be a simple algebra of classical type. If $\operatorname{rank}(\mathfrak{g}) \leq 2$ then $\hat{\mathfrak{g}}$ has a complete set of restricted Sugawara fields.

Proof. Since $\mathfrak{g}$ is of classical type, the basic invariants for $\mathfrak{g}$ have degrees at most 4 . The basic invariants of degree $<4$ give restricted Sugawara fields by Theorem 4.1(a). If $\mathfrak{g}$ admits a degree $\dot{4}$ basic invariant $u$, we apply Corollary 4.3 to replace $u$ by $u^{\#}$, which is algebraically independent of the lower-order basic invariants and gives a restricted Sugawara field.

For the remainder of this section we take $\mathfrak{g}=\operatorname{sl}_{n}(C)$ and $G=\mathrm{SU}(n)$. The ring of $G$-invariants in $S(\mathfrak{g})$ is a polynomial ring on $n-1$ generators. In this section we construct a specific set of generators that furnish a complete set of restricted Sugawara fields for $\hat{\mathfrak{g}}$.

Theorem 4.11. Let $X \in \mathfrak{g}$. Assume that $X$ satisfies equations (12)-(15) of $\S 4.2$. Set

$$
u_{k}=\int_{G}\left(X^{g}\right)^{k} d g
$$

Then the following hold:

(i) $\sigma\left(u_{k}\right)(\zeta)$ is a restricted Sugawara field on every module in the category $\mathscr{F}$, for $k=2, \ldots, n$.

(ii) $u_{2}, \ldots, u_{n}$ are algebraically independent generators of $S(\mathfrak{g})^{G}$.

Part (i) of the theorem follows immediately from Corollary 4.9 and Theorem 4.4. The rest of this section will be devoted to the proof of part (ii) of the theorem. We shall identify $S(\mathfrak{g})$ and $S\left(\mathfrak{g}^{*}\right)$ as $G$-modules using the trace form $B$ on $C^{n}$, as in $\S 4.2$. The basic strategy is to express the invariants $u_{k}$ in terms of the invariants $s_{k}$ using formulas of Frobenius and Harish-Chandra. For this we must first recall some facts about the finite-dimensional holomorphic representations of $\mathrm{SL}_{n}(\mathrm{C})$ (cf. [Wey]).

Let $H \subset \mathrm{SL}_{n}(\mathbf{C})$ be the subgroup of diagonal matrices, and let $\mathfrak{h}$ be its Lie algebra. Let $\Lambda_{+}$be the set of all

$$
\lambda=\left(m_{1}, m_{2}, \ldots, m_{n}\right) \in \mathbf{N}^{n}, \quad \text { with } m_{1} \geq m_{2} \geq \cdots \geq m_{n} \geq 0
$$

Each such $\lambda$ defines a dominant integral linear functional on the diagonal matrices in $\mathrm{gl}_{n}(\mathbf{C})$ by the formula

$$
\lambda(X)=m_{1} x_{1}+\cdots+m_{n} x_{n}
$$


if $X=\operatorname{diag}\left(x_{1}, \ldots, x_{n}\right)$. Let $\chi_{\lambda}$ be the character of the irreducible finitedimensional holomorphic representation of $\mathrm{GL}_{n}(\mathbf{C})$ with highest weight $\lambda$. Then $\chi_{\lambda}$ is a polynomial function on $\mathrm{GL}_{n}(\mathbf{C})$, relative to the matrix coordinates. Hence it extends to a polynomial function on $M_{n}(\mathrm{C})$ and by restriction gives a polynomial function on $\mathfrak{g}$.

Let $\Lambda_{++}$be the set of all $\mu=\left(\mu_{1}, \ldots, \mu_{n}\right) \in \mathbf{N}^{n}$ such that

$$
\mu_{1}>\mu_{2}>\cdots>\mu_{n} \geq 0 \text {. }
$$

Define $\rho=(n-1, n-2, \ldots, 1,0) \in \Lambda_{++}$. If $\mu \in \Lambda_{++}$, then $\mu=\lambda+\rho$, with $\lambda \in \Lambda_{+}$. For $\lambda=\left(m_{1}, m_{2}, \ldots, m_{n}\right) \in \Lambda_{+}$, set

$$
|\lambda|=m_{1}+\cdots+m_{n}, \quad \lambda !=\left(m_{1} !\right) \cdots\left(m_{n} !\right) .
$$

Let $\Lambda_{+}(k)=\left\{\lambda \in \Lambda_{+}:|\lambda|=k\right\}$. If $\lambda \in \Lambda_{+}(k)$, then $\chi_{\lambda}$ is a $G$-invariant polynomial function homogeneous of degree $k$ on $\mathfrak{g}$.

Lemma 4.12. Let $X \in \mathfrak{g}$. Then

$$
\int_{G}\left(X^{g}\right)^{k} d g=\gamma_{n, k} \sum_{\lambda \in \Lambda_{+}(k)} \frac{1}{(\lambda+\rho) !} \chi_{\lambda}(X) \chi_{\lambda}
$$

for $k=1,2, \ldots$, where $\gamma_{n, k}$ is a nonzero constant not depending on $X$. Here the left side of (2) is viewed as a polynomial function on $\mathfrak{g}$ via the form $B$.

Proof. Given $X=\operatorname{diag}\left(x_{1}, \ldots, x_{n}\right)$, we set

$$
\pi(X)=\prod_{i<j}\left(x_{i}-x_{j}\right) .
$$

Say $X$ is regular if $\pi(X) \neq 0$. It will suffice to prove the lemma for regular $X \in \mathfrak{h}$, since both sides of (2) are invariant polynomial functions of $X$. Let $W=\Sigma_{n}$, the symmetric group on $n$ letters, acting on $\mathfrak{h}$ by permuting the coordinates. For $w \in W$ let $\varepsilon(w)$ be the sign of $w$. For regular elements $X, Y \in \mathfrak{h}$, one has Harish-Chandra's orbital Fourier transform formula

$$
\int_{G} e^{t B\left(X^{g}, Y\right)} d g=\frac{\gamma t^{-r}}{\pi(X) \pi(Y)} \sum_{w \in W} \varepsilon(w) e^{t B(w X, Y)}
$$

where $t \in \mathbf{C}, r=n(n-1) / 2$ is the number of positive roots of $(\mathfrak{g}, \mathfrak{h})$, and $\gamma$ is a nonzero constant depending only on $n$ [Har]. Expanding both sides of (3) and equating the coefficients of $t^{k}$, we obtain

$$
\frac{1}{k !} \int_{G} B\left(X^{g}, Y\right)^{k} d g=\frac{\gamma}{(k+r) ! \pi(X) \pi(Y)} \sum_{w \in W} \varepsilon(w) B(w X, Y)^{k+r} .
$$

Let $X=\operatorname{diag}\left(x_{1}, \ldots, x_{n}\right)$ and $Y=\operatorname{diag}\left(y_{1}, \ldots, y_{n}\right)$. For $\mu=\left(\mu_{1}, \ldots, \mu_{n}\right)$ $\in \mathbf{N}^{n}$, define

$$
A^{\mu}(X)=\operatorname{det}\left[x_{i}^{\mu_{j}}\right]_{1 \leq i, j \leq n} .
$$


Expanding $B(w X, Y)^{k+r}$ by the multinomial formula, we obtain

$$
\sum_{w \in W} \varepsilon(w) B(w X, Y)^{k+r}=\sum_{|\mu|=k+r} \frac{1}{\mu !} A^{\mu}(X) \prod_{i=1}^{n} y_{i}^{\mu_{i}}
$$

where the summation is over $\mu \in \mathbf{N}^{n}$. Notice that $A^{\mu}(X)=0$ if any of the components of $\mu$ are equal. If the components of $\mu$ are distinct, then there are a unique permutation $w \in W$ and a dominant integral $\lambda \in \Lambda_{+}(k)$ so that $w \mu=\lambda+\rho$. We can thus write the right side of (5) as

$$
\sum_{\lambda \in \Lambda_{+}(k)} \sum_{w \in W} \frac{1}{(\lambda+\rho) !} A^{w \cdot(\lambda+\rho)}(X) \prod_{i=1}^{n} y_{i}^{(w \cdot(\lambda+\rho))_{i}} .
$$

Since $A^{\mu}(X)=\varepsilon(w) A^{w \mu}(X)$, the sum over $W$ of the products involving $Y$ gives $A^{\lambda+\rho}(Y)$. Hence

$$
\sum_{w \in W} \varepsilon(w) B(w X, Y)^{k+r}=\sum_{\lambda \in \Lambda_{+}(k)} \frac{1}{(\lambda+\rho) !} A^{\lambda+\rho}(X) A^{\lambda+\rho}(Y) .
$$

By the Weyl character formula we have

$$
\chi_{\lambda}(X)=A^{\lambda+\rho}(X) / \pi(X)
$$

for $X \in \mathfrak{g}$ regular. (Recall that we have extended $\chi_{\lambda}$ from $G$ to $\mathfrak{g}$ using matrix coordinates, and that in these coordinates the function $\pi(X)$ is the same as the Weyl denominator for $G$.) The lemma now follows from (4), (6), and (7), since the polynomial function on $\mathfrak{g}$ corresponding to $X^{k}$ is $Y \rightarrow B(Y, X)^{k}$.

We now recall the duality between the irreducible representations of the symmetric group $\Sigma_{k}$ and those of $\mathrm{GL}_{n}(\mathbf{C})$ (cf. [Wey] and [Mur]). We assume $k \leq n$. Given $\lambda=\left(m_{1}, \ldots, m_{n}\right) \in \Lambda_{+}(k)$, we let $Y_{\lambda}$ be the associated Young diagram with $i$ th row having $m_{i}$ boxes. Let $\chi^{\lambda}$ be the character of the irreducible representation of $\Sigma_{k}$ associated with the Young symmetrizer for $Y_{\lambda}$. For example, if $\lambda=(k, 0, \ldots, 0)$, then $\chi^{\lambda}(w)=1$. If $\lambda=(1,1, \ldots, 1,0, \ldots, 0)$, then $\chi^{\lambda}(w)=\varepsilon(w)$.

Let $p=\left(p_{1}, p_{2}, \ldots\right) \in \mathscr{P}_{k}$ be a partition of $k$. We denote by $C^{p}$ the conjugacy class in $\Sigma_{k}$ consisting of all elements having $p_{i}$ cycles of length $i$. (This class is often denoted by $1^{p_{1}} 2^{p_{2}} \ldots$ in the literature.) We denote by $C_{k}$ the conjugacy class of elements having a single cycle of length $k$. For any conjugacy class $C$ in $\Sigma_{k}$, we write $\chi^{\lambda}(C)$ for the value of the character $\chi^{\lambda}$ on the class $C$ and $\operatorname{Card}(C)$ for the number of elements in $C$.

Lemma 4.13. Let $X \in \mathfrak{g}$. Then for $k=2,3, \ldots, n$ one has

$$
\int_{G}\left(X^{g}\right)^{k} d g=J_{k}(X) s_{k} \quad \bmod \mathbf{C}\left[s_{2}, \ldots, s_{k-1}\right] \text {. }
$$

Here

$$
J_{k}(X)=\gamma_{n, k} \sum_{\lambda \in \Lambda_{+}(k)} \frac{1}{(\lambda+\rho) !} \chi^{\lambda}\left(C_{k}\right) \chi_{\lambda}(X),
$$

where $\gamma_{n, k}$ is a nonzero constant not depending on $X$. 
Proof. Let $\lambda \in \Lambda_{+}(k)$. By a formula of Frobenius [Wey, equation 7.19], one has

$$
\chi_{\lambda}=\frac{1}{k !} \sum_{p \in \mathscr{P}_{k}} \operatorname{Card}\left(C^{p}\right) \chi^{\lambda}\left(C^{p}\right) s^{p} .
$$

The lemma follows by using (10) in the right side of (2) and observing that the invariant $s_{k}$ only occurs in connection with the conjugacy class $C_{k}$.

Lemma 4.14. Let $X \in \mathfrak{g}$ and suppose $X$ satisfies equations (12)-(15) of $\S 4.2$. Then $J_{k}(X)$ in Lemma 4.13 is a nonzero multiple of

$$
\sum_{\lambda \in \Lambda_{+}(k)} \sum_{p \in \mathscr{T}_{k}} \frac{(-n)^{|p|}}{(\lambda+\rho) !} \operatorname{Card}\left(C^{p}\right)\left\{\prod_{i \geq 2}(i-1)^{p_{i}}\right\} \chi^{\lambda}\left(C_{k}\right) \chi^{\lambda}\left(C^{p}\right)
$$

for $2 \leq k \leq n$.

Proof. Formula (11) follows immediately from (9), (10), and formula (16) of $\S 4.2$.

Proof of Theorem 4.11(ii). Recall that $s_{2}, \ldots, s_{n}$ are algebraically independent generators for $S(\mathfrak{g}){ }^{G}$. Hence by Lemma 4.13 we only need to prove that the leading coefficients $J_{k}(X) \neq 0$ for $k=2, \ldots, n$, because we can then recursively express $\left\{s_{k}\right\}$ in terms of $\left\{u_{k}\right\}$. If we denote the quantity (11) as $f_{k}(n)$, then by Lemma 4.14 we are reduced to proving that $f_{k}(n) \neq 0$ for $k=2, \ldots, n$. This turns out to be remarkably difficult because of the extensive cancellations that occur in the formula defining $f_{k}(n)$. We shall make a detailed analysis of the terms in (11) and derive another formula for $f_{k}(n)$ in which all the terms are of the same sign.

For $2 \leq k \leq n$ set

$$
\lambda_{0}=(k, 0, \ldots, 0) \in \Lambda_{+}(k),
$$

and for $1 \leq j \leq k-1$, let

$$
\lambda_{j}=(k-j, 1, \ldots, 1,0, \ldots, 0) \in \Lambda_{+}(k),
$$

where 1 occurs as the second to the $(j+1)$ th component of $\lambda_{j}$. By [Mur, equation 5.3] one knows that if $\lambda \in \Lambda_{+}(k)$, then

$$
\chi^{\lambda}\left(C_{k}\right)= \begin{cases}(-1)^{j} & \text { if } \lambda=\lambda_{j} \\ 0 & \text { otherwise }\end{cases}
$$

For $0 \leq j \leq k-1$, define

$$
\alpha_{j, k}(n)=(-1)^{j} \sum_{p \in \mathscr{P}_{k}} \operatorname{Card}\left(C^{p}\right)(-n)^{|p|}\left\{\prod_{i \geq 2}(i-1)^{p_{i}}\right\} \chi^{\lambda_{j}}\left(C^{p}\right) .
$$


From (11) and (13) we see that

$$
f_{k}(n)=\sum_{j=0}^{k-1} \frac{1}{\left(\lambda_{j}+\rho\right) !} \alpha_{j, k}(n) .
$$

We now proceed to prove that $f_{k}(n) \neq 0$. This requires a rather lengthy argument, because the terms on the right side of (15) are not all of the same sign. Recall that $\lambda_{0}$ parametrizes the trivial representation of $\Sigma_{k}$. Since

$$
\operatorname{Card}\left(C^{p}\right)=\frac{k !}{p_{1} ! p_{2} ! \cdots} \frac{1}{1^{p_{1}} 2^{p_{2}} \cdots}
$$

for $p$ a partition of $k$, we can write equation (14) in the case $j=0$ as

$$
\frac{1}{k !} \alpha_{0, k}(n)=\sum_{p \in \mathscr{P}_{k}} \prod_{i \geq 2}\left\{\frac{(-n)^{p_{i}}}{p_{i} !}\left(\frac{i-1}{i}\right)^{p_{i}}\right\} .
$$

Note in particular that $\alpha_{0, k}(n)$ is a polynomial function of $n$ and hence is defined for any complex value of $n$.

We set $\alpha_{0,0}(n)=1$ and form the generating function

$$
A(n, x)=\sum_{k=0}^{\infty} \frac{x^{k}}{k !} \alpha_{0, k}(n)
$$

with $|x|<1$. From formula (17) we calculate that

$$
\begin{aligned}
A(n, x) & =1+\sum_{p \in \mathscr{D}} \prod_{i \geq 1} \frac{1}{p_{i} !}\left(\frac{n(1-i) x^{i}}{i}\right)^{p_{i}} \\
& =\prod_{i=1}^{\infty} \exp \left\{\frac{n(1-i) x^{i}}{i}\right\} \\
& =\exp \left\{n \sum_{i=1}^{\infty} \frac{x^{i}}{i}\right\} \exp \left\{-n \sum_{i=1}^{\infty} x^{i}\right\} .
\end{aligned}
$$

Thus we have

$$
A(n, x)=(1-x)^{-n} \exp \left\{-\frac{n x}{1-x}\right\}
$$

for $|x|<1$. Since $A(-n, x) A(n, x)=1$, we obtain the orthogonality relation

$$
\sum_{i=0}^{k}\left(\begin{array}{l}
k \\
i
\end{array}\right) \alpha_{0, i}(-n) \alpha_{0, k-i}(n)=0
$$

for $k \geq 1$, because the left side of (19) is the coefficient of $x^{k} / k$ ! in the series expansion of $A(-n, x) A(n, x)$.

In general, for $0 \leq j \leq k-1$ and $p$ a partition of $k$, we have from Proposition A.4 in the appendix that

$$
(-1)^{j} \chi^{\lambda_{j}}\left(C^{p}\right)=\sum_{0 \leq w(q) \leq j}(-1)^{|q|} \prod_{i \geq 2}\left(\begin{array}{c}
p_{i} \\
q_{i}
\end{array}\right) .
$$


The sum in (20) is over all $q \in \mathscr{P}$ such that $0 \leq w(q) \leq j$ and $q_{i} \leq p_{i}$ for $i=1,2, \ldots$. Substituting (16) and (20) in (14) and rearranging terms, we find by (17) that

$$
\alpha_{j, k}(n)=\sum_{i=0}^{j}\left(\begin{array}{c}
k \\
i
\end{array}\right) \alpha_{0, i}(-n) \alpha_{0, k-i}(n)
$$

for $0 \leq j \leq k-1$.

Next, we calculate the factorial term in $f_{k}(n)$ as follows:

$$
\begin{aligned}
\left(\lambda_{j}+\rho\right) ! & =(k-j+n-1) !(n-1) !(n-2) ! \cdots(n-j) !(n-j-2) ! \cdots 1 ! \\
& =\frac{\Gamma(n+k-j)}{\Gamma(n-j)} \prod_{i=1}^{n-1}(n-i) !
\end{aligned}
$$

where $\Gamma(x)$ is Euler's gamma function. By the integral formula for the beta function, we have

$$
\frac{\Gamma(n-j)}{\Gamma(n+k-j)}=\Gamma(k)^{-1} \int_{0}^{1} x^{n-j-1}(1-x)^{k-1} d x .
$$

Thus we can write

$$
\frac{1}{\left(\lambda_{j}+\rho\right) !}=\frac{1}{M} \int_{0}^{1} x^{n-j-1}(1-x)^{k-1} d x,
$$

where $M=(k-1) ! \prod_{i=1}^{n-1}(n-i)$ ! is positive and does not depend on $j$.

Now we substitute (21) and (22) in (15):

$$
f_{k}(n)=\frac{1}{M} \int_{0}^{1}\left\{\sum_{j=0}^{k-1} \sum_{i=0}^{j}\left(\begin{array}{c}
k \\
i
\end{array}\right) \alpha_{0, i}(-n) \alpha_{0, k-i}(n) x^{n-j-1}\right\}(1-x)^{k-1} d x .
$$

Interchanging the order of summation on $i$ and $j$ and summing the resulting finite geometric series, we find that

$$
f_{k}(n)=\frac{1}{M} \int_{0}^{1} \sum_{i=0}^{k-1}\left(\begin{array}{c}
k \\
i
\end{array}\right) \alpha_{0, i}(-n) \alpha_{0, k-i}(n)\left(1-x^{k-i}\right) x^{n-k}(1-x)^{k-2} d x
$$

Notice that because the factor $\left(1-x^{k-i}\right)$ vanishes when $i=k$, we may change the upper limit of summation to $k$ in formula (23). Having done this, we can then replace the factor $\left(1-x^{k-i}\right)$ by $-x^{k-i}$, because of the orthogonality relation (19). Thus if we define

$$
b_{n, k}(x)=\sum_{i=0}^{k}\left(\begin{array}{c}
k \\
i
\end{array}\right) \alpha_{0, i}(-n) \alpha_{0, k-i}(n) x^{k-i}
$$

then we can write

$$
f_{k}(n)=-\frac{1}{M} \int_{0}^{1} b_{n, k}(x) x^{n-k}(1-x)^{k-2} d x .
$$


We observe that the measure $x^{n-k}(1-x)^{k-2} d x$ in integral (25) is positive. Hence to prove $f_{k}(n) \neq 0$, it will suffice to prove that $b_{n, k}(x)>0$ in $0<x<1$. We shall do this by finding a differential-difference equation satisfied by $b_{n, k}(x)$.

Form the generating function

$$
B_{n}(t, x)=\sum_{k=0}^{\infty} \frac{t^{k}}{k !} b_{n, k}(x) .
$$

From (24) we see that

$$
B_{n}(t, x)=A(-n, t) A(n, t x) .
$$

Hence by formula (18) we.calculate that

$$
\frac{\partial}{\partial x} B_{n}(t, x)=-\frac{n t^{2} x}{(1-t x)^{2}} B_{n}(t, x) \text {. }
$$

But

$$
\frac{n t^{2} x}{(1-t x)^{2}}=-n \sum_{j=2}^{\infty}(j-1) t^{j} x^{j-1}
$$

By multiplying the series (26) and (28), we find the coefficient of $t^{k} / k$ ! in the Taylor expansion of the right side of (27) to be

$$
-n \sum_{i=0}^{k-2} \frac{k !(k-i-1)}{i !} x^{k-i-1} b_{n, i}(x) .
$$

Thus we obtain the differential-difference equation

$$
\frac{\partial}{\partial x} b_{n, k}(x)=-n \sum_{i=0}^{k-2} \frac{k !(k-i-1)}{i !} x^{k-i-1} b_{n, i}(x) .
$$

Since $b_{n, 0}(x)=1$ and $b_{n, k}(1)=0$ for all $k \leq n$ by (19), we conclude by (29) and induction on $k$ that

$$
b_{n, k}(x)>0 \text { and } \frac{\partial}{\partial x} b_{n, k}(x)<0 \text { for } 0<x<1 \text { and } 2 \leq k \leq n .
$$

This completes the proof.

\section{SUgAWARA OPERATORS AND CHARACTER FORMULAS}

5.1. Generalized Harish-Chandra homomorphism. Throughout this section we assume that $\mathfrak{g}$ is a finite-dimensional simple Lie algebra over $\mathbf{C}$, and we take $B$ to be the Killing form. We recall some notation from [Wal]. Fix $\mathfrak{h}$, a Cartan subalgebra of $\mathfrak{g}$, and let $\Phi$ be the root system of $\mathfrak{g}$ with respect to $\mathfrak{h}$. For $\alpha \in \Phi$, we denote by $\mathfrak{g}_{\alpha}$ the corresponding root space. Fix a system $\Phi^{+}$of positive roots in $\Phi$. Let $\alpha_{1}, \ldots, \alpha_{l}$ be the set of simple roots in $\Phi^{+}$and let $\beta$ denote the largest root. We set

$$
\mathfrak{n}_{0}=\sum_{\alpha \in \Phi^{+}} \mathfrak{g}_{\alpha}, \quad \overline{\mathfrak{n}}_{0}=\sum_{\alpha \in \Phi^{+}} \mathfrak{g}_{-\alpha} .
$$


Choose, for $\alpha \in \Phi$, elements $X_{\alpha} \in \mathfrak{g}_{\alpha}$ such that $\alpha\left(\left[X_{\alpha}, X_{-\alpha}\right]\right)=2$. Put $h_{\alpha}=\left[X_{\alpha}, X_{-\alpha}\right]$. If we set, for $i=1, \ldots, l$,

$$
\begin{array}{lll}
e_{i}=X_{\alpha_{i}}, & f_{i}=X_{-\alpha_{i}}, & h_{i}=h_{\alpha_{i}}, \\
e_{0}=X_{-\beta}(1), & f_{0}=X_{\beta}(-1), & h_{0}=-h_{\beta}+2 c /(\beta, \beta),
\end{array}
$$

then we obtain a standard set of generators for the affine algebra $\hat{\mathfrak{g}}$. Set

$$
\hat{\mathfrak{h}}=\sum_{i=0}^{l} \mathbf{C} h_{i}, \quad \hat{\mathfrak{h}}^{e}=\mathbf{C} d_{0} \oplus \hat{\mathfrak{h}} .
$$

Let $\delta$ and $\rho$ in $\left(\hat{\mathfrak{h}}^{e}\right)^{*}$ be defined by $\delta(\hat{\mathfrak{h}})=0, \delta\left(d_{0}\right)=1$ and $\rho\left(d_{0}\right)=0$, $\rho\left(h_{i}\right)=1$ for $i=0,1, \ldots, l$.

For $\alpha \in\left(\hat{\mathfrak{h}}^{e}\right)^{*}$ let $\hat{\mathfrak{g}}_{\alpha}=\left\{x \in \hat{\mathfrak{g}}:[H, x]=\alpha(H) x\right.$ for $\left.H \in \hat{\mathfrak{h}}^{e}\right\}$. We denote by $\Delta=\left\{\alpha \neq 0: \hat{\mathfrak{g}}_{\alpha} \neq 0\right\}$ the roots of $\hat{\mathfrak{h}}^{e}$ on $\hat{\mathfrak{g}}$. Then

$$
\Delta=\{\alpha+n \delta: \alpha \in \Phi, n \in \mathbf{Z}\} \cup\{n \delta: n \in \mathbf{Z}-\{0\}\},
$$

where we extend $\alpha \in \Phi$ to $\hat{\mathfrak{h}}^{e}$ by $\alpha(c)=\alpha\left(d_{0}\right)=0$. We set

$$
\begin{aligned}
& \Delta_{R}^{+}=\{\alpha+n \delta: \alpha \in \Phi, n=1,2, \ldots\} \cup \Phi^{+}, \\
& \Delta_{I}^{+}=\{n \delta: n=1,2, \ldots\} .
\end{aligned}
$$

The system of positive roots for $\hat{\mathfrak{h}}^{e}$ is then $\Delta^{+}=\Delta_{R}^{+} \cup \Delta_{I}^{+}$. We define subalgebras

$$
\mathfrak{n}=\sum_{\alpha \in \Delta^{+}} \hat{\mathfrak{g}}_{\alpha}, \quad \mathfrak{v}=\sum_{\alpha \in \Delta^{+}} \hat{\mathfrak{g}}_{-\alpha} .
$$

Then we have the triangular decomposition $\hat{\mathfrak{g}}^{e}=\mathfrak{v} \oplus \hat{\mathfrak{h}}^{e} \oplus \mathfrak{n}$.

On the loop algebra $\mathfrak{g} \otimes \mathbf{C}\left[z, z^{-1}\right]$ we put the nondegenerate invariant bilinear form $B\left(X \otimes z^{p}, Y \otimes z^{q}\right)=\delta_{p+q, 0} B(X, Y)$. We extend $B$ to an invariant form on $\hat{\mathfrak{g}}^{e}$ by setting $B(c, c)=0, B\left(d_{0}, d_{0}\right)=0$, and $B\left(c, d_{0}\right)=1$. The restriction of this form to $\hat{\mathfrak{h}}^{e}$ is nondegenerate, and we write $(\cdot, \cdot)$ for the dual form on $\left(\hat{\mathfrak{h}}^{e}\right)^{*}$.

Let $\mathscr{O}$ be the category of all finitely generated $\hat{\mathfrak{g}}^{e}$ modules $M$ such that

(i) $\hat{\mathfrak{h}}^{\boldsymbol{e}}$ acts semisimply on $M$;

(ii) if $m \in M$ then there exists a positive integer $k$ such that $\mathfrak{n}^{k} m=0$.

In particular, modules in $\mathscr{O}$ satisfy the u-finiteness condition (1) of $\S 2.2$ and hence are in the category $\mathscr{F}$. Given $\Lambda \in\left(\hat{\mathfrak{h}}^{e}\right)^{*}$, we write $\mathbf{C}_{\Lambda}$ for the one-dimensional $\hat{\mathfrak{h}}^{e} \oplus \mathfrak{n}$ module with $\hat{\mathfrak{h}}^{e}$ acting by $\Lambda$ and $\mathfrak{n}$ acting by 0 . We define

$$
M(\Lambda)=U\left(\hat{\mathfrak{g}}^{e}\right) \otimes_{U\left(\hat{\mathfrak{h}}^{e} \oplus \mathfrak{n}\right)} \mathbf{C}_{\Lambda}
$$

The module $M(\Lambda) \in \mathscr{O}$ and has a unique irreducible quotient that we denote by $L(\Lambda)$.

We shall need to analyze the modules $M(\Lambda)$ for varying $\Lambda$. Since

$$
U\left(\hat{\mathfrak{g}}^{e}\right)=U(\mathfrak{v}) \otimes U\left(\hat{\mathfrak{h}}^{e} \oplus \mathfrak{n}\right)
$$


by the Poincaré-Birkhoff-Witt (PBW) theorem, we may realize the module $M(\Lambda)$ as the fixed vector space $U(\mathfrak{v})$ with an action $\pi_{\Lambda}$ of $\hat{\mathfrak{g}}^{e}$ that depends on $\Lambda$. Under this identification the element $1 \otimes 1$ will be denoted as $v_{\Lambda}$. If $Y \in \mathfrak{v}$, then $\pi_{\Lambda}(Y)$ is left multiplication by $Y$ and is constant in $\Lambda$.

Let $u \in S(\mathfrak{g})$, and consider the formal operator $\sigma(u)(\zeta)$ on $M(\Lambda)$, as defined in Lemma 2.3. For $\Lambda \in \hat{\mathfrak{h}}^{e}$ there is a unique element $z_{u}(\Lambda)(\zeta) \in U(\mathfrak{v})\{\zeta\}$ such that

$$
\sigma(u)(\zeta) v_{\Lambda}=z_{u}(\Lambda)(\zeta) v_{\Lambda}
$$

If $u$ has degree $n$, then $z_{u}(\Lambda)(\zeta)$ is a polynomial in $\Lambda$ of degree at most $n$. We shall determine the leading terms in this polynomial when $u$ is $G$-invariant.

There is a projection $p: U(\mathfrak{g}) \rightarrow U(\mathfrak{h})$ defined by the decomposition

$$
U(\mathfrak{g})=U(\mathfrak{h}) \oplus\left\{\mathfrak{n}_{0} U(\mathfrak{g})+U(\mathfrak{g}) \overline{\mathfrak{n}}_{0}\right\} .
$$

Let $\omega: S(\mathfrak{g}) \rightarrow U(\mathfrak{g})$ be the canonical symmetrization map. We shall denote by

$$
\boldsymbol{\beta}: S(\mathfrak{g}) \rightarrow U(\mathfrak{h})
$$

the composite map $\boldsymbol{\beta}(u)=p(\omega(u))$. If $\Lambda \in \hat{\mathfrak{h}}^{e}$, we write $\Lambda_{0}$ for the restriction of $\Lambda$ to $\mathfrak{h}$. Fix an orthonormal basis $\left\{H_{i}\right\}$ for $\mathfrak{h}$ and let the gradient operators $\nabla_{i}$ on $S(\mathfrak{g})$ be defined as in $\S 1.1$ relative to this basis.

Lemma 5.1. If $u \in S^{n}(\mathfrak{g})^{G}$, then

$$
\begin{aligned}
z_{u}(\Lambda)(\zeta)= & \beta(u)\left(\Lambda_{0}\right)+\sum_{i=1}^{l} \nabla_{i} u\left(\Lambda_{0}\right)\left(H_{i}\right)_{-}(\zeta) \\
& +\{\text { terms of degree } \leq n-2 \text { in } \Lambda\}
\end{aligned}
$$

Proof. It will suffice to prove (2) when

$$
u=\int_{G}\left(X^{g}\right)^{n} d g
$$

for some $X \in \mathfrak{g}$, since elements of this form span $S^{n}(\mathfrak{g})^{G}$. We start by studying the dependence of $: X(\zeta)^{n}: v_{\Lambda}$ on $\Lambda$. We first observe that $\pi_{\Lambda}\left(X_{+}(\zeta)\right)^{k} v_{\Lambda}=$ $\pi_{\Lambda}(X)^{k} v_{\Lambda}$, since $v_{\Lambda}$ is annihilated by $\pi_{\Lambda}(X(j))$ for $j>0$ and $[X, X(j)]=0$. But from the commutation relations in $\mathfrak{g}$ we calculate that

$$
\pi_{\Lambda}(X)^{k} v_{\Lambda}=B\left(X, H_{\Lambda}\right)^{k} v_{\Lambda}+\{\text { terms of degree } \leq k-1 \text { in } \Lambda\},
$$

where $H_{\Lambda} \in \mathfrak{h}$ corresponds to $\Lambda_{0}$ via the form $B$. The operator $\pi_{\Lambda}\left(X_{-}(\zeta)\right)^{k}$ does not depend on $\Lambda$. We now combine these observations with the normalordered binomial expansion (Lemma 2.3) to obtain

$$
\begin{aligned}
\sigma\left(X^{n}\right)(\zeta) v_{\Lambda}= & \pi_{\Lambda}(X)^{n} v_{\Lambda}+n B\left(X, H_{\Lambda}\right)^{n-1} \pi_{\Lambda}\left(X_{-}(\zeta)\right) v_{\Lambda} \\
& +\{\text { terms of degree } \leq n-2 \text { in } \Lambda\} .
\end{aligned}
$$


We can write $\pi_{\Lambda}(X)^{n} v_{\Lambda}=\pi_{\Lambda}\left(\omega\left(X^{n}\right)\right) v_{\Lambda}$, where $\omega$ is the symmetrization map. Replacing $X$ by $X^{g}$ in (3) and integrating over $G$, we have

$$
\begin{aligned}
\sigma(u)(\zeta) v_{\Lambda}= & \pi_{\Lambda}(\omega(u)) v_{\Lambda}+\int_{G} n B\left(X^{g}, H_{\Lambda}\right)^{n-1} \pi_{\Lambda}\left(\left(X^{g}\right)_{-}(\zeta)\right) v_{\Lambda} d g \\
& +\{\text { terms of degree } \leq n-2 \text { in } \Lambda\} .
\end{aligned}
$$

But by the usual formula for the Harish-Chandra homomorphism, $\pi_{\Lambda}(\omega(u)) v_{\Lambda}$ $=\boldsymbol{\beta}(u)\left(\Lambda_{0}\right)$. Furthermore, we can expand

$$
\pi_{\Lambda}\left(\left(X^{g}\right)_{-}(\zeta)\right)=\sum_{i=1}^{l} B\left(X^{g}, H_{i}\right) \pi_{\Lambda}\left(\left(H_{i}\right)_{-}(\zeta)\right) .
$$

Since

$$
\nabla_{i} u\left(\Lambda_{0}\right)=\int_{G} n B\left(X^{g}, H_{\Lambda}\right)^{n-1} B\left(X^{g}, H_{i}\right) d g,
$$

we can thus rewrite (4) to obtain equation (2).

5.2. Character formulas for category $\mathscr{O}_{I}$. We define the subcategory $\mathscr{O}_{I}=\{M \in$ $\mathscr{O}: c+\rho(c)=0$ on $M$ \}. Recall from [Wal, Lemma 1.1] that $\rho(c)=\frac{1}{2}$ and that the module $M(\Lambda)$ is in $\mathscr{O}_{I}$ if and only if

$$
(\Lambda+\rho, \gamma)=0 \text { for all } \gamma \in \Delta_{I}
$$

(the shifted highest weight $\Lambda+\rho$ is on the boundary of the Tits cone; cf. [Kac]). When $u \in S(\mathfrak{g})^{G}$ and $\sigma(u)(\zeta)$ is a restricted Sugawara field, then $\sigma(u)(\zeta)$ commutes with the action of $\hat{\mathfrak{g}}$ on any module in the category $\mathscr{O}_{I}$.

In this section we shall assume the existence of a complete set of restricted Sugawara fields for $\hat{\mathfrak{g}}$ and derive an explicit character formula for "generic" irreducible modules in the category $\mathscr{O}_{I}$. This extends the results in [Wal], where the case $\mathfrak{g}=\mathrm{sl}(2, \mathrm{C})$ was treated. By Theorems 4.10 and 4.11 this completeness assumption is true for $g$ of type $B_{2}$ and $A_{n}, n=1,2,3, \ldots$.

If $\Lambda \in \mathfrak{h}^{*}$ then we extend $\Lambda$ to $\hat{\mathfrak{h}}^{e}$ by setting $\Lambda(c)=-\frac{1}{2}$ and $\Lambda\left(d_{0}\right)=0$. With this definition, we have a map $\Lambda \rightarrow M(\Lambda)$ from $\mathfrak{h}^{*}$ to $\mathscr{O}_{I}$. For $\alpha \in \mathfrak{h}^{*}$ let $H_{\alpha} \in \mathfrak{h}$ be defined by $B\left(X, H_{\alpha}\right)=\alpha(X)$ for all $X \in \mathfrak{h}$. Denote by

$$
\bar{\omega}=\prod_{\alpha \in \Phi^{+}} H_{\alpha}
$$

the fundamental skew-invariant element in $S(\mathfrak{h})$. Define

$$
D_{R}=\prod_{\alpha \in \Delta_{R}^{+}}\left(1-e^{-\alpha}\right) .
$$

Theorem 5.2. Assume that $\sigma\left(u_{1}\right)(\zeta), \ldots, \sigma\left(u_{l}\right)(\zeta)$ is a complete set of restricted Sugawara fields for $\hat{\mathfrak{g}}$. Set $T_{i}(\zeta)=\sigma\left(u_{i}\right)(\zeta)$ and write

$$
T_{i}(\zeta)=\sum_{n \in \mathbf{Z}} T_{i}(n) \zeta^{n}
$$


There exists a countable set of rational functions $\left\{\phi_{i}\right\}$ on $\mathfrak{h}^{*}$ such that

(1) The singularities of the $\phi_{i}$ are contained in $\left\{\Lambda \in \mathfrak{h}^{*}: \bar{\omega}(\Lambda)=0\right\}$.

(2) If $\Lambda \in \mathfrak{h}^{*}$ and $\bar{\omega}(\Lambda) \neq 0$, then the function $t \rightarrow \phi_{i}(t \Lambda)$ is not identically zero.

(3) Set $\Omega=\left\{\Lambda \in \mathfrak{h}^{*}: \phi_{i}(\Lambda) \neq 0\right.$ for $\left.i=1,2, \ldots\right\}$. If $\Lambda \in \Omega$ then

(a) $L(\Lambda)=M(\Lambda) /\left\{\sum_{i=1}^{l} \sum_{n=1}^{\infty} T_{i}(-n) M(\Lambda)\right\}$;

(b) $\operatorname{ch} L(\Lambda)=e^{\Lambda} / D_{R}$;

(c) $\operatorname{Hom}_{\hat{\mathfrak{g}}}(M(\Lambda), M(\Lambda))$ is the algebra generated by the set of mutually commuting operators $\left\{T_{i}(-n): 1 \leq i \leq l, n=1,2, \ldots\right\}$.

We shall prove this theorem following the same general lines as [Wal, Theorem 2.5]. It will require several preliminary results, however, that we develop in the next two sections.

5.3. Decompositions of $U(\mathfrak{v})$. Let the invariants $u_{i}$ and the operators $T_{i}(n)$ be as in Theorem 5.2. Then $\left[d_{0}, T_{i}(n)\right]=n T_{i}(n)$ on $M(\Lambda)$. Hence $T_{i}(n)=0$ for $n>0$ since the spectrum of $\pi_{\Lambda}\left(d_{0}\right)$ is $\{0,-1, \ldots\}$. For $\Lambda \in \mathfrak{h}^{*}$ we can thus expand

$$
z_{u_{i}}(\Lambda)(\zeta)=\sum_{n \in \mathbf{N}} z_{i}(\Lambda)(-n) \zeta^{-n}
$$

where $z_{i}(\Lambda)(-n) \in U(\mathfrak{v})$ is of degree $-n$ relative to $d_{0}$.

Lemma 5.3. Let $\Lambda \in \mathfrak{h}^{*}$. Then for $k, m \in \mathbf{N}$ and $i, j=1, \ldots, l$

$$
\left[T_{i}(-k), T_{j}(-m)\right]=0
$$

on $M(\Lambda)$. For any $H \in \mathfrak{h},\left[H, z_{i}(\Lambda)(-k)\right]=0$. Furthermore

$$
\left[z_{i}(\Lambda)(-k), z_{j}(\Lambda)(-m)\right]=0
$$

in $U(\mathfrak{v})$.

Proof. By Lemma 2.1, $\left[T_{i}(-k), X(n)\right]=0$ as operators on $M$ for all $X \in$ $\mathfrak{g}$ and $n \in \mathbf{Z}$. But for any vector $v \in M$ there exists $y \in U(\hat{\mathfrak{g}})$ so that $T_{j}(-m) v=y v$. Hence (1) holds. To prove (2), write

$$
\begin{aligned}
T_{i}(-k) T_{j}(-m) v_{\Lambda} & =T_{i}(-k) z_{j}(\Lambda)(-m) v_{\Lambda} \\
& =z_{j}(\Lambda)(-m) T_{i}(-k) v_{\Lambda} \\
& =z_{j}(\Lambda)(-m) z_{i}(\Lambda)(-k) v_{\Lambda} .
\end{aligned}
$$

Hence by (1) we have

$$
z_{i}(\Lambda)(-k) z_{j}(\Lambda)(-m) v_{\Lambda}=z_{j}(\Lambda)(-m) z_{i}(\Lambda)(-k) v_{\Lambda} .
$$

But the map $y \rightarrow \pi_{\Lambda}(y) v_{\Lambda}$ is injective on $U(\mathfrak{v})$, so (2) follows. The same argument, with $T_{j}(-m)$ replaced by $H$ or $d_{0}$, shows that $\left[H, z_{i}(-k)\right] v_{\Lambda}=0$ and hence the commutator is zero, since $[\mathfrak{h}, \mathfrak{v}] \subset \mathfrak{v}$.

Let $H_{1}, \ldots, H_{l}$ be a fixed orthonormal basis for $\mathfrak{h}$ and set

$$
H_{i}(\Lambda)=\sum_{j=1}^{l} \nabla_{j} u_{i}(\Lambda) H_{j}
$$


for $\Lambda \in \mathfrak{h}^{*}$. Since $u_{1}, \ldots, u_{l}$ are basic invariants, we know that $\operatorname{det}\left[\nabla_{j} u_{i}(\Lambda)\right]$ is a nonzero multiple of $\bar{\omega}(\Lambda)$. Hence $H_{1}(\Lambda), \ldots, H_{l}(\Lambda)$ define a basis of $\mathfrak{h}$ if $\bar{\omega}(\Lambda) \neq 0$. We set $H_{i}(\Lambda)(n)=H_{i}(\Lambda) \otimes z^{n}$. Let $d_{i}=\operatorname{deg}\left(u_{i}\right)$. Then $H_{i}(\Lambda)(n)$ is homogeneous of degree $d_{i}-1$ in $\Lambda$.

Let $H \in \mathfrak{h}$ satisfy $\alpha_{i}(H)=1$ for $i=1,2, \ldots, l$. The subalgebra $\mathfrak{v}$ is stable under ad $H$ and decomposes as

$$
\mathfrak{v}=\mathfrak{v}^{-} \oplus \mathfrak{v}^{0} \oplus \mathfrak{v}^{+}
$$

into negative, zero, and positive eigenspaces for ad $H$. We have

$$
\begin{aligned}
\mathfrak{v}^{-} & =\overline{\mathfrak{n}}_{0} \otimes \mathbf{C}\left[z^{-1}\right], \\
\mathfrak{v}^{0} & =\mathfrak{h} \otimes \mathbf{C}\left[z^{-1}\right] z^{-1}, \\
\mathfrak{v}^{+} & =\mathfrak{n}_{0} \otimes \mathbf{C}\left[z^{-1}\right] z^{-1} .
\end{aligned}
$$

The summands in (3) are subalgebras and satisfy $\left[\mathfrak{v}^{0}, \mathfrak{v}^{ \pm}\right] \subset \mathfrak{v}^{ \pm}$. Let $d=$ $\operatorname{dim} \mathfrak{n}_{0}$.

For defining various PBW bases, we shall need to use the sets $\mathscr{I}, \mathcal{J}$, and $\mathscr{K}$ of multi-indices of the form

$$
\begin{array}{lll}
I=\left(I_{i n}\right), & 1 \leq i \leq l, & n=1,2, \ldots, \\
J=\left(J_{p m}\right), & 1 \leq p \leq d, & m=1,2, \ldots, \\
K=\left(K_{p m}\right), & 1 \leq p \leq d, & m=0,1, \ldots,
\end{array}
$$

respectively, with the indices nonnegative integers and all but a finite number of them zero. We write $I_{i}=\left\{I_{i n}\right\}$ for fixed $i$. We then form three sets of operators $\left\{T^{I}\right\},\left\{H(\Lambda)^{I}\right\}$, and $\left\{z(\Lambda)^{I}\right\}$ indexed by $\mathscr{I}$, with $\Lambda \in \mathfrak{h}^{*}$ appearing as a parameter, as follows:

$$
T^{I}=T_{1}^{I_{1}} T_{2}^{I_{2}} \ldots \quad \text { where } T_{i}^{I_{i}}=T_{i}(-1)^{I_{i 1}} T_{i}(-2)^{I_{i 2}} \ldots
$$

The other products are defined similarly by replacing $T_{i}(-n)$ with $H_{i}(\Lambda)(-n)$ or $z_{i}(\Lambda)(-n)$. Note that since the operators in each family mutually commute (Lemma 5.3), the ordering of the products is immaterial. let

Pick a basis $X_{1}, \ldots, X_{d}$ for $\mathfrak{n}_{0}$ such that ad $H\left(X_{p}\right)=n_{p} X_{p}$. For $J \in \mathcal{J}$

$$
X^{J}=X_{1}^{J_{1}} X_{2}^{J_{2}} \cdots \quad \text { where } X_{i}^{J_{i}}=X_{i}(-1)^{J_{i 1}} X_{i}(-2)^{J_{i 2}} \cdots
$$

Then by the PBW theorem $\left\{X^{J}: J \in \mathcal{J}\right\}$ is a basis for $U\left(\mathfrak{v}^{+}\right)$. We similarly fix a basis $Y_{1}, \ldots, Y_{d}$ for $\bar{n}_{0}$ such that ad $H\left(Y_{p}\right)=-n_{p} Y_{p}$. By the PBW theorem, we obtain a basis for $U\left(\mathfrak{v}^{-}\right)$indexed by $\mathscr{K}$ consisting of the ordered monomials

$$
Y^{K}=Y_{1}^{K_{1}} Y_{2}^{K_{2}} \cdots \quad \text { where } Y_{i}^{K_{i}}=Y_{i}^{K_{i 0}} Y_{i}(-1)^{K_{i 1}} Y_{i}(-2)^{K_{i 2}} \cdots
$$

Let $d_{i}=\operatorname{deg}\left(u_{i}\right)$. For $I \in \mathscr{I}$ and $K \in \mathscr{K}$ we set

$$
\mu(I)=\sum_{i, n}\left(d_{i}-1\right) I_{i n}, \quad \nu(K)=\sum_{p, n} n_{p} K_{p n} .
$$


Because $d_{i} \geq 2$ and $n_{p} \geq 1$ in these sums, the sets $\{I \in \mathscr{I}: \mu(I)=r\}$ and $\{K \in \mathscr{K}: \nu(K)=r\}$ are finite for each positive integer $r$.

From (3) and the PBW theorem, there is a direct sum decomposition

$$
U(\mathfrak{v})=U\left(\mathfrak{v}^{0} \oplus \mathfrak{v}^{-}\right) \oplus \mathfrak{v}^{+} U(\mathfrak{v}) .
$$

Let $q: U(\mathfrak{v}) \rightarrow U\left(\mathfrak{v}^{0} \oplus \mathfrak{v}^{-}\right)$be the corresponding projection.

Lemma 5.4. There exists a countable set of polynomials $\left\{\phi_{j}\right\}$ on $\mathfrak{h}^{*}$ satisfying the properties (1) and (2) of Theorem 5.2, such that if $\phi_{j}(\Lambda) \neq 0$ for all $j$ then

$$
q: \bigoplus_{I \in \mathcal{I}} U\left(\mathfrak{v}^{-}\right) z^{I}(\Lambda) \rightarrow U\left(\mathfrak{v}^{-} \oplus \mathfrak{v}^{0}\right)
$$

is a linear isomorphism.

Proof. Let $H \in \mathfrak{h}$ be as above, with $\alpha_{i}(H)=1$ for $i=1, \ldots, l$. Then $q(\operatorname{ad} H \cdot x)=\operatorname{ad} H \cdot q(x)$. We grade $U\left(\mathfrak{v}^{-}\right)$relative to ad $H$ by

$$
U^{(m)}\left(\mathfrak{v}^{-}\right)=\left\{x \in U\left(\mathfrak{v}^{-}\right): \operatorname{ad}(H) x=-m x\right\}
$$

for $m \in \mathbf{N}$. Then $U^{(0)}\left(\mathfrak{v}^{-}\right)=\mathbf{C}$ and $U^{(m)}\left(\mathfrak{v}^{-}\right)$has basis $\left\{Y^{K}: K \in \mathscr{K}, \nu(K)=\right.$ $m\}$ for $m>0$. Hence $U^{(m)}\left(\mathfrak{b}^{-}\right)$is finite-dimensional. We shall prove that

$$
q: \sum_{\mu(I)=i} U^{(k)}\left(\mathfrak{v}^{-}\right) z^{I}(\Lambda) \rightarrow \sum_{\mu(I)=i} U^{(k)}\left(\mathfrak{v}^{-}\right) H(\Lambda)^{I}
$$

is a linear isomorphism for all $i, k \in \mathbf{N}$, subject to the nonvanishing of polynomials $\left\{\phi_{j}(\Lambda)\right\}$ satisfying (1), (2) of Theorem 5.2.

For $i=0,(4)$ is the identity map on $U^{(k)}\left(\mathfrak{v}^{-}\right)$, so the result is true in this case. Let $i>0$ and $k \geq 0$. Fix a linear order on the finite set

$$
M_{i, k}=\{(I, K): I \in \mathscr{I}, \mu(I)=i \text { and } K \in \mathscr{K}, \nu(K)=k\}
$$

and use this order to arrange the elements $Y^{k} z^{I}(\Lambda)$ (resp. $\left.Y^{K} H(\Lambda)^{I}\right)$ into a column vector

$$
B_{i, k}(\Lambda)=\left[Y^{K} z^{I}(\Lambda)\right]_{I, K \in M_{i k}} \quad\left(\text { resp. } C_{i, k}(\Lambda)=\left[Y^{K} H(\Lambda)^{I}\right]_{I, K \in M_{i k}}\right) .
$$

Assume that $\bar{\omega}(\Lambda) \neq 0$. Since $\left[H, z^{I}(\Lambda)\right]=0$, we can write

$$
q\left(B_{i, k}(\Lambda)\right)=A_{i, k}(\Lambda) C_{i, k}(\Lambda)
$$

where $A_{i, k}(\Lambda)$ is a matrix with rational entries whose singularities are contained in $\left\{\Lambda \in \mathfrak{h}^{*}: \bar{\omega}(\Lambda)=0\right\}$. The lemma will be proved if we show that the function $t \rightarrow \operatorname{det} A_{i, k}(t \Lambda)$, for $t \in \mathbf{C}$, is not identically zero when $\bar{\omega}(\Lambda) \neq 0$. We can then take for the functions in the lemma the set $\left\{\operatorname{det} A_{i, k}(\Lambda): i>0, k \geq 0\right\}$.

By Lemma 5.1,

$$
z_{j}(t \Lambda)(-n)=t^{d_{j}-1} H_{j}(\Lambda)(-n)+\{\text { lower order in } t\}
$$

for all $n>0$. Hence for all $I \in \mathscr{J}$

$$
z^{I}(t \Lambda)=t^{\mu(I)} H(\Lambda)^{I}+\{\text { lower order in } t\} .
$$


Thus $B_{i, k}(t \Lambda)=t^{i} C_{i, k}(\Lambda)+\{$ lower order in $t\}$. Since $C_{i, k}(t \Lambda)=t^{i} C_{i, k}(\Lambda)$, it follows from the definition of the matrix $A_{i, k}(\Lambda)$ that $A_{i, k}(t \Lambda)=$ Identity + terms of order $t^{-1}$. Hence

$$
\lim _{t \rightarrow \infty} A_{i, k}(t \Lambda)=\text { Identity }
$$

This completes the proof.

Let $\left\{\phi_{i}\right\}$, be as in Lemma 5.4. Set

$$
\Omega=\left\{\Lambda \in \mathfrak{h}^{*}: \bar{\omega}(\Lambda) \neq 0 \text { and } \phi_{i}(\Lambda) \neq 0 \text { for all } i\right\} .
$$

Let $\mathscr{A}$ be the ring of operators on $M(\Lambda)$ generated by $\left\{T_{i}(-n): 1 \leq i \leq l, n=\right.$ $1,2, \ldots\}$. By Lemma $5.3, \mathscr{A}$ is commutative.

Lemma 5.5. If $\Lambda \in \Omega$ then $M(\Lambda)$ is a free $\mathscr{A}$ module on $U\left(\mathfrak{v}^{+}\right) U\left(\mathfrak{v}^{-}\right) v_{\Lambda}$.

Proof. By the PBW theorem, $\left\{X^{J} Y^{K}: J \in \mathcal{J}\right.$ and $\left.K \in \mathscr{K}\right\}$ is a basis for $U\left(\mathfrak{v}^{+}\right) U\left(\mathfrak{v}^{-}\right)$. Since the operators $T^{I}$, for $I \in \mathscr{I}$, commute with the action of $\hat{\mathfrak{g}}$, we have

$$
T^{I} X^{J} Y^{K} v_{\Lambda}=X^{J} Y^{K} z^{I}(\Lambda) v_{\Lambda}
$$

Hence it will suffice to prove that for $\Lambda \in \Omega$

$$
\left\{X^{J} Y^{K} z^{I}(\Lambda): I \in \mathscr{I}, J \in \mathscr{J}, K \in \mathscr{K}\right\}
$$

is a basis for $U(\mathfrak{b})$.

We grade $U(\mathfrak{v})$ by the homogeneous derivation $d$ that acts by $d \cdot e_{i}=e_{i}$ and $d \cdot f_{i}=-f_{i}$ for $i=0,1, \ldots, l$. Define

$$
U(\mathfrak{b})_{[j]}=\{x \in U(\mathfrak{b}): d \cdot x=-j x\}
$$

for $j=0,1,2, \ldots$. These subspaces are finite-dimensional. Let

$$
\mathscr{S}_{j}=\left\{(I, J, K): X^{J} Y^{K} z^{I}(\Lambda) \in U(\mathfrak{v})_{[j]}\right\} \text {. }
$$

We shall prove by induction on $j$ that the elements $X^{J} Y^{K} z^{I}(\Lambda)$ for $(I, J, K)$ $\in \mathscr{S}_{j}$ are a basis for $U(\mathfrak{v})_{[j]}$.

If $j=0$ then $U(\mathfrak{v})_{[0]}=\mathbf{C} \cdot 1$ and the result is true. Assume that the result is true for $j<r$. If $v \in U(\mathfrak{b})_{[r]}$ then by Lemma 5.4 there exists $a_{I, K} \in \mathbf{C}$ so that the element

$$
w=\sum_{(I, 0, K) \in \mathscr{S}_{j}} a_{I, K} Y^{K} z^{I}(\Lambda)
$$

satisfies $q(w)=q(v)$. Thus there exist elements $w_{i, n} \in U(\mathfrak{v})_{[j]}$, with $j<r$, such that

$$
v-w=\sum_{i=1}^{d} \sum_{n=1}^{\infty} X_{i}(-n) w_{i, n} .
$$

Now applying the induction hypothesis to $w_{i, n}$ we can write $X_{i}(-n) w_{i, n}$ as a sum of elements $X_{i}(-n) X^{J} Y^{K} z^{I}(\Lambda)$ of total degree $p$ relative to the derivation 
$d$. We can then rearrange the factors $X_{i}(-n) X^{J}$ as a sum of terms $X^{J^{\prime}}$ without changing the total degree. This proves that $X^{J} Y^{K} z^{I}(\Lambda)$ for $(I, K, J) \in \mathscr{S}_{r}$ span $U(\mathfrak{v})_{[r]}$. But obviously the elements $X^{J} Y^{K} H(\Lambda)^{I}$ for $(I, K, J) \in \mathscr{S}_{r}$ are a basis for $U(\mathfrak{v})_{[r]}$. Hence by dimension, the set

$$
\left\{X^{J} Y^{K} z^{I}(\Lambda):(I, K, J) \in \mathscr{S}_{r}\right\}
$$

is linearly independent. This completes the induction step.

5.4. Filtrations on $M(\Lambda)$. Recall that there is an anti-automorphism $x \rightarrow x^{t}$ of $\hat{\mathfrak{g}}^{e}$ that is the identity on $\hat{\mathfrak{h}}^{e}$ and that interchanges the canonical generators $e_{i}$ and $f_{i}(i=0,1, \ldots, l)$. There is a canonical bilinear form $(\cdot, \cdot)_{\Lambda}$ on $M(\Lambda)$ that satisfies

$$
\left(\pi_{\Lambda}(x) y, z\right)_{\Lambda}=\left(y, \pi_{\Lambda}\left(x^{t}\right) z\right)_{\Lambda}
$$

(the contravariant form). We view this as a form varying with $\Lambda$ on the fixed vector space $U(\mathfrak{v})$.

Let $\mathbf{C}[t] \otimes U(\mathfrak{v})$ be looked upon as the space of all polynomials in one variable with values in $U(\mathfrak{v})$. Set

$$
\begin{array}{r}
M(\Lambda)_{j}=\left\{x(0) v_{\Lambda}: x \in \mathbf{C}[t] \otimes U(\mathfrak{v}) \text { with }\left(x(t) v_{\Lambda}, z\right)_{\Lambda+t \rho} \in t^{j} \mathbf{C}[t]\right. \\
\text { for all } z \in M(\Lambda)\} .
\end{array}
$$

Then $M(\Lambda)=M(\Lambda)_{0} \supset M(\Lambda)_{1} \supset \cdots$ defines a $\hat{\mathfrak{g}}$-invariant filtration of $M(\Lambda)$ called the "Jantz : filtration" (cf. [K-K]). If $I \in \mathscr{I}$, then let $|I|=\sum I_{\text {in }}$.

Lemma 5.6. If $\Lambda \in \mathfrak{h}^{*}$ and $I \in \mathscr{I}$, then $T^{I} v_{\Lambda} \in M(\Lambda)_{|I|}$.

Proof. This follows by the same argument as Lemma 2.2 in [Wal].

We shall compare the Jantzen filtration with the following filtration of $M(\Lambda)$. For $j \geq 0$ set

$$
N(\Lambda)_{j}=\sum_{|I| \geq j} U(\hat{\mathfrak{g}}) T^{I} v_{\Lambda} .
$$

Then $M(\Lambda)=N(\Lambda)_{0} \supset N(\Lambda)_{1} \supset \cdots$ defines a $\hat{\mathfrak{g}}$-invariant filtration of $M(\Lambda)$, and $M(\Lambda)_{j} \supset N(\Lambda)_{j}$ by Lemma 5.6. We now calculate the character of the module $N(\Lambda)_{j}$. Set

$$
D_{R}=\prod_{\alpha \in \Delta_{R}^{+}}\left(1-e^{-\alpha}\right) .
$$

For $I$ in $\mathscr{J}, \mathscr{J}$, or $\mathscr{K}$ we set $w(I)=\sum n I_{\text {in }}$. Define

$$
p_{l, j}(n)=\operatorname{Card}\{I \in \mathscr{I}: w(I)=n \text { and }|I| \geq j\} .
$$

Lemma 5.7. If $\Lambda \in \Omega$ then

$$
\operatorname{ch} N(\Lambda)_{j}=\frac{e^{\Lambda}}{D_{R}} \sum_{n \geq 1} p_{l, j}(n) e^{-n \delta}
$$


Proof. By Lemma 5.5, $N(\Lambda)_{j}$ has a basis

$$
\left\{T^{I} X^{J} Y^{K} v_{\Lambda}: I \in \mathscr{J}, J \in \mathscr{J}, K \in \mathscr{K},|I| \geq j\right\} .
$$

For $h \in \hat{\mathfrak{h}}^{e}$ we have $\left[h, T^{I} X^{J} Y^{K}\right]=-\eta(h) T^{I} X^{J} Y^{K}$, with

$$
\eta=\sum_{i=1}^{l}\left\{w\left(I_{i}\right) \delta+w\left(J_{i}\right) \delta\right\}-\sum_{i=1}^{d}\left\{\left|J_{i}\right| \alpha_{i}+w\left(K_{i}\right) \delta+\left|K_{i}\right| \alpha_{i}\right\}
$$

Here $I_{i}=\left(I_{i 1}, I_{i 2}, \ldots\right)$ and similarly with $J_{i}$ and $K_{i}$. It follows that

$$
\begin{aligned}
\operatorname{ch} N(\Lambda)_{j}=e^{\Lambda}\left\{\sum_{J \in \mathcal{J}} \prod_{i=1}^{d} e^{-w\left(J_{i}\right) \delta+\left|J_{i}\right| \alpha_{i}}\right\} \\
\cdot\left\{\sum_{K \in \mathscr{K}} \prod_{i=1}^{d} e^{-w\left(K_{i}\right) \delta-\left|K_{i}\right| \alpha_{i}}\right\} \sum_{n \geq 1} p_{l, j}(n) e^{-n \delta} .
\end{aligned}
$$

To obtain formula (1), we show that the product of two factors involving the roots of $\mathfrak{g}$ on the right side of (2) gives $1 / D_{R}$. To see this, let $\mathbf{N}_{m} \subset \mathbf{N}^{m}$ be the $m$-tuples of nonnegative integers $\left(p_{1}, \ldots, p_{m}\right)$ with $p_{m} \geq 1$. For $L \in \mathbf{N}_{m}$ set $w(L)=\sum n p_{n}$. Then the first bracketed term on the right in (2) is the product over all roots $\alpha \in \Phi^{+}$of the sum

$$
1+\sum_{m=1}^{\infty} \sum_{L \in \mathbf{N}_{m}} e^{-w(L) \delta+|L| \alpha}
$$

But (3) can be expressed as

$$
1+e^{-\delta+\alpha} \sum_{m=1}^{\infty} \prod_{n=1}^{m}\left(1-e^{-n \delta+\alpha}\right)^{-1}
$$

which can be factored as

$$
\prod_{n=1}^{\infty}\left(1-e^{-n \delta+\alpha}\right)^{-1}
$$

A similar calculation shows that the second bracketed term on the right in (2) is the product over all roots $\alpha \in \Phi^{+}$of

$$
\prod_{n=0}^{\infty}\left(1-e^{-n \delta-\alpha}\right)^{-1}
$$

By the description of $\Delta_{R}^{+}$in $\S 5.1$, we conclude that the product of the two bracketed terms on the right in (2) is $1 / D_{R}$.

5.5. Proof of Theorem 5.2. We now assemble the results of the previous sections to prove Theorem 5.2. The first step is to show that the two filtrations introduced in $\$ 5.4$ coincide when $\Lambda$ is in suitably general position. We start with Jantzen's character sum formula (cf. [K-K]).

$$
\sum_{j \geq 1} \operatorname{ch} M(\Lambda)_{j}=\sum_{\alpha \in \tilde{\Delta}^{+}} \sum_{\substack{j \geq 1 \\ L_{j n}(\Lambda)=0}} \operatorname{ch} M(\Lambda-j \alpha) .
$$


Here $\tilde{\Delta}^{+}$is the set of positive roots of $\hat{\mathfrak{g}}$ with each root repeated according to its multiplicity, and $L_{\mu}(\Lambda)=2(\Lambda+\rho, \mu)-(\mu, \mu)$. We assume that $\Lambda$ satisfies $L_{j \alpha}(\Lambda) \neq 0$ for all integers $j>0$ and all $\alpha \in \Delta_{R}^{+}$. Then only the imaginary roots $r \delta, r>0$, occur on the right side of $(1)$, with multiplicity $l$. (Recall that we are assuming that $\Lambda \in \mathfrak{h}$ and hence $(\Lambda, \delta)=0$.) It follows that

$$
\sum_{j \geq 1} \operatorname{ch} M(\Lambda)_{j}=l \sum_{\substack{j \geq 1 \\ r \geq 1}} \operatorname{ch} M(\Lambda-j r \delta) .
$$

For $n$ a positive integer, set $\sigma(n)=\operatorname{Card}\{(p, q) \in \mathbf{N} \times \mathbf{N}: p q=n\}$. Then (2) can be written as

$$
\sum_{j \geq 1} \operatorname{ch} M(\Lambda)_{j}=l \sum_{n \geq 1} \sigma(n) \operatorname{ch}(M(\Lambda-n \delta)) .
$$

For any $\mu \in \hat{\mathfrak{h}}^{e}$ the character of the module $M(\mu)$ is

$$
\operatorname{ch} M(\mu)=e^{\mu} / D_{R} D_{I}
$$

where

$$
\frac{1}{D_{I}}=\sum_{n=0}^{\infty} p_{l}(n) e^{-n \delta}=\prod_{k=1}^{\infty}\left(1-e^{-k \delta}\right)^{-l}
$$

Here $p_{l}(n)=\operatorname{Card}\{I \in \mathscr{I}: w(I)=n\}$. It follows that

$$
\begin{aligned}
\sum_{j \geq 1} \operatorname{ch} M(\Lambda)_{j} & =\frac{l}{D_{R}} \sum_{n \geq 1} \sigma(n) e^{\Lambda-n \delta} \sum_{k \geq 0} p_{l}(k) e^{-k \delta} \\
& =\frac{l}{D_{R}} \sum_{n \geq 1} \sum_{k \geq 0} \sigma(n) p_{l}(k) e^{\Lambda-(n+k) \delta} .
\end{aligned}
$$

We now need the following combinatorial result, generalizing [Wal, Lemma 2.4].

Lemma 5.8. One has

$$
l \sum_{i \geq 1} p_{l}(n-i) \sigma(i)=\sum_{j \geq 1} p_{l, j}(n) .
$$

Proof. For $\sigma(n)$ we have the generating function

$$
\sum_{n \geq 0} \sigma(n) q^{n}=\sum_{p \geq 1} \frac{q^{p}}{1-q^{p}}
$$

It follows that

$$
\sum_{n \geq 0} q^{n}\left\{\sum_{i \geq 1} p_{l}(n-i) \sigma(i)\right\}=\prod_{k=1}^{\infty}\left(1-q^{k}\right)^{-l} \sum_{p \geq 1} \frac{q^{p}}{1-q^{p}} .
$$

Now introduce formal variables $x_{i, n}$, for $i=1, \ldots, l$ and $n=1,2, \ldots$. Let

$$
E=\sum_{i, n} x_{i, n} \frac{\partial}{\partial x_{i, n}}
$$


be the formal Euler operator. Then

$$
E \prod_{\substack{1 \leq i \leq l \\ n>0}}\left(1-x_{i, n}\right)^{-1}=\prod_{\substack{1 \leq i \leq l \\ n>0}}\left(1-x_{i, n}\right)^{-1} \sum_{\substack{1 \leq i \leq l \\ n>0}} \frac{x_{i, n}}{1-x_{i, n}} .
$$

On the other hand, we can expand

$$
\prod_{\substack{1 \leq i \leq l \\ n>0}}\left(1-x_{i, n}\right)^{-1}=\sum_{I \in \mathcal{I}} x^{I}
$$

from which we obtain

$$
E \prod_{\substack{1 \leq i \leq l \\ n>0}}\left(1-x_{i, n}\right)^{-1}=\sum_{I \in \mathcal{F}}|I| x^{I}
$$

Comparing this with (6) and specializing to $x_{i, n}=q^{n}$, we have the identity

$$
l \prod_{k=1}^{\infty}\left(1-q^{k}\right)^{-l} \sum_{p \geq 1} \frac{q^{p}}{1-q^{p}}=\sum_{n \geq 1}\left\{\sum_{I \in \mathcal{S}_{n}}|I|\right\} q^{n} .
$$

So comparing (5) and (7), we conclude that for $n \geq 1$,

$$
\sum_{i \geq 1} p_{l}(n-i) \sigma(i)=\sum_{I \in \mathscr{I}_{n}}|I| \text {. }
$$

On the other hand,

$$
\operatorname{Card}\{I \in \mathscr{I}:|I|=j\}=p_{l, j}(n)-p_{l, j+1}(n) .
$$

Hence

$$
\sum_{I \in \mathcal{I}_{n}}|I|=\sum_{j \geq 1} j\left(p_{l, j}(n)-p_{l, j+1}(n)\right)=\sum_{j \geq 1} p_{l, j}(n) .
$$

This proves the lemma.

At this point we can describe the set $\left\{\phi_{j}\right\}$ in Theorem 5.2: take the functions $\phi_{j}$ in Lemma 5.4 together with all the functions $L_{j \alpha}$ with $j=1,2, \ldots$ and $\alpha \in \Delta_{R}^{+}$. Define the generic set $\Omega$ by these functions. If $\Lambda \in \Omega$ then by Lemmas 5.7 and 5.8 we have

$$
\sum_{j \geq 1} \operatorname{ch} N(\Lambda)_{j}=\sum_{j \geq 1} \operatorname{ch} M(\Lambda)_{j}
$$

Since $N(\Lambda)_{j} \subset M(\Lambda)_{j}$ it follows that $N(\Lambda)_{j}=M(\Lambda)_{j}$ for all $j$. In particular, $N(\Lambda)_{1}=M(\Lambda)_{1}$, which proves part (3a) of the theorem. Part (3b) follows from Lemma 5.5. To obtain (3c), we need an additional lemma.

Lemma 5.9. If $\Lambda \in \Omega$ then for $j \geq 1$

$$
N(\Lambda)_{j} / N(\Lambda)_{j+1} \cong \bigoplus_{n \geq 1}\left(p_{l, j}(n)-p_{l, j+1}(n)\right) L(\Lambda-n \delta)
$$

as a $\hat{\mathfrak{g}}^{e}$ module. 
Proof. By Lemma 5.5,

$$
N(\Lambda)_{j} / N(\Lambda)_{j+1}=\bigoplus_{\substack{I \in \mathcal{I} \\|I|=j}} T^{I} U\left(\mathfrak{v}^{+}\right) U\left(\mathfrak{v}^{-}\right) v_{\Lambda} .
$$

Thus by (8) and part (3b) of Theorem 5.2, we have

$$
\operatorname{ch} N(\Lambda)_{j} / N(\Lambda)_{j+1}=\sum_{n \geq 1}\left(p_{l, j}(n)-p_{l, j+1}(n)\right) \operatorname{ch} L(\Lambda-n \delta) .
$$

We fix $j$ and write

$$
v^{I}(\Lambda)=T^{I} v_{\Lambda}+N(\Lambda)_{j+1} \text { for } I \in \mathscr{I}
$$

with $|I|=j$. Then $v^{I}(\Lambda)$ is a nonzero highest-weight vector of weight $\Lambda$ $w(I) \delta$ in $N(\Lambda)_{j} / N(\Lambda)_{j+1}$. By Lemma 5.5 the set $\left\{v^{I}(\Lambda): I \in \mathscr{I},|I|=j\right\}$ is linearly independent. Since

$$
\operatorname{ch}\left(\bigoplus_{|I|=j} \operatorname{Cv}^{I}(\Lambda)\right) \operatorname{ch} L(\Lambda)=\operatorname{ch}\left(N(\Lambda)_{j} / N(\Lambda)_{j+1}\right)
$$

by (9), we conclude that

$$
\left(N(\Lambda)_{j} / N(\Lambda)_{j+1}\right)^{\mathrm{n}}=\bigoplus_{|I|=j} \mathrm{C}^{I}(\Lambda)
$$

We now assert that if $|I|=j$ then

$$
U(\hat{\mathfrak{g}}) v^{I}(\Lambda) \cong L(\Lambda-w(I) \delta) .
$$

Indeed, the submodule $U(\hat{\mathfrak{g}}) T^{I} v_{\Lambda}$ of $M(\Lambda)$ is isomorphic to $M(\Lambda-w(I) \delta)$, since the map $y \rightarrow \pi_{\Lambda}(y) v^{I}(\Lambda)$ is injective on $U(\mathfrak{v})$. But the operators $T_{i}(-n)$ are functorially defined on any module in $\mathscr{O}_{I}$. Hence by part (3a) of Theorem 5.2 , we conclude that

$$
\sum_{i=1}^{l} \sum_{n=1}^{\infty} T_{i}(-n) U(\hat{\mathfrak{g}}) T^{I} v_{\Lambda}
$$

is the maximal proper submodule in $U(\hat{\mathfrak{g}}) T^{I} v_{\Lambda}$. This submodule is contained in $N(\Lambda)_{j+1}$, so $(11)$ is an isomorphism.

Since the highest-weight vectors $v^{I}(\Lambda)$ are independent and the modules $U(\hat{\mathfrak{g}}) v^{I}(\Lambda)$ are irreducible, it follows from (10) that

$$
N(\Lambda)_{j} / N(\Lambda)_{j+1}=\bigoplus_{|I|=j} U(\hat{\mathfrak{g}}) v^{I}(\Lambda)
$$

This proves the lemma.

We can now prove part (3c) of Theorem 5.2. Suppose that

$$
S \in \operatorname{Hom}_{\hat{\mathfrak{g}}}(M(\Lambda), M(\Lambda)) .
$$


We may assume that $\left[d_{0}, S\right]=-k S$ for some integer $k>0$. Set $z_{S}=S v_{\Lambda}$, and pick $j$ so that $z_{S} \in N(\Lambda)_{j}$ but $z_{S} \notin N(\Lambda)_{j+1}$. Since $z_{S}$ is annihilated by $\mathfrak{n}$, it follows from Lemma 5.9 that there are scalars $a_{I, j}$ such that

$$
z_{S}-\sum_{\substack{|I|=j \\ w(I)=k}} a_{I, j} T^{I} v_{\Lambda} \in N(\Lambda)_{j+1} .
$$

Thus replacing $S$ by $\sum a_{I, j} T^{I}$, we may assume that $z_{S} \in N(\Lambda)_{j+1}$. After a finite number of steps we get $z_{S}=0$ and hence $S=0$, since the map $S \rightarrow z_{S}$ is injective on $\operatorname{Hom}_{\hat{\mathfrak{g}}}(M(\Lambda), M(\Lambda))$. This means that the original $S$ is a polynomial in the operators $T^{I}$.

\section{APPENDIX}

A.1. Characters of some representations of $\Sigma_{k}$. We shall derive the formula for the characters of $\Sigma_{k}$ associated with the highest weights $\lambda_{j}$ of $\mathrm{GL}_{n}(\mathbf{C})$ defined in $\S 4.3$, formula (12). Recall that the Young diagram corresponding to $\lambda_{j}$ is an inverted "L" with $j+1$ rows. The first row has length $k-j$ and the remaining rows have length 1 . We shall write

$$
\chi_{j, k}=\chi_{\lambda_{j}}
$$

for the character of the representation of $\mathrm{GL}_{n}(\mathbf{C})$ with highest weight $\lambda_{j}$, for $j=0,1, \ldots, k-1$. This is defined provided $1 \leq k \leq n$.

For $j=0$ and $k \geq 1$ we shall write $\chi_{0, k}=q_{k}$, following the notation of classical invariant theory (cf. [Mur]). This is the character of the representation of $\mathrm{GL}_{n}(\mathbf{C})$ on $S^{k}\left(\mathbf{C}^{n}\right)$, which is paired with the trivial representation of $\Sigma_{k}$ in the Frobenius-Schur-Weyl theory. Thus by formula (10) of $\S 4.3$ one has

$$
q_{k}=\frac{1}{k !} \sum_{p \in \mathscr{P}_{k}} \operatorname{Card}\left(C^{p}\right) s^{p}
$$

for $k=1,2, \ldots$ We define $q_{0}=1$ and $q_{k}=0$ for $k<0$.

When $j=k-1$, then $\chi_{k-1, k}$ is the character of the representation of $\mathrm{GL}_{n}(\mathbf{C})$ on the $k$ th exterior power $\wedge^{k} \mathbf{C}^{n}$. This representation is paired with the signum representation of $\Sigma_{k}$. If $p \in \mathscr{P}_{k}$ is a partition, define

$$
\sigma(p)=\sum_{i} p_{2 i}
$$

Then on the conjugacy class $C^{p} \subset \Sigma_{k}$ the signum representation has value $(-1)^{\sigma(p)}$. We shall write $\Theta_{k}=\chi_{k-1, k}$. By formula (10) of $\S 4.3$ one has

$$
\boldsymbol{\Theta}_{k}=\frac{1}{k !} \sum_{p \in \mathscr{P}_{k}} \operatorname{Card}\left(C^{p}\right)(-1)^{\sigma(p)} s^{p}
$$

for $k=1,2, \ldots, n$. 
Lemma A.1. One has

$$
\chi_{j, k}=q_{k-j} \boldsymbol{\Theta}_{j}-\chi_{j-1, k}
$$

for $1 \leq j \leq k$.

Proof. By Jacobi's formula (cf. [Mur, equation 4.35]) one has

$$
\chi_{j, k}=\operatorname{det}\left[\begin{array}{ccccc}
q_{k-j} & q_{k-j+1} & q_{k-j+2} & \cdots & q_{k} \\
1 & q_{1} & q_{2} & \cdots & q_{j} \\
0 & 1 & q_{1} & \cdots & q_{j-1} \\
\cdot & \cdot & \cdot & \cdot & \cdot \\
0 & \cdot & \cdot & 1 & q_{1}
\end{array}\right]
$$

Note that the matrix in $(4)$ is $(j+1)$ by $(j+1)$. Expanding the determinant by the first column, we obtain

$$
\begin{aligned}
\chi_{j, k}= & q_{k-j} \operatorname{det}\left[\begin{array}{cccc}
q_{1} & q_{2} & \cdots & q_{j} \\
1 & q_{1} & \cdots & q_{j-1} \\
\cdot & \cdot & \cdot & \cdot \\
0 & \cdots & 1 & q_{1}
\end{array}\right] \\
& -\operatorname{det}\left[\begin{array}{cccc}
q_{k-j+1} & q_{k-j+2} & \cdots & q_{k} \\
1 & q_{1} & \cdots & q_{j-1} \\
\cdot & \cdot & \cdot & \cdot \\
0 & \cdots & 1 & q_{1}
\end{array}\right] .
\end{aligned}
$$

Comparing this expansion with (4), we obtain (3).

Fix $k \geq 1$. We shall write $\chi^{j}=\chi^{\lambda_{j}}$ for the character of $\Sigma_{k}$ that is dual to $\chi_{j, k}$. As we have already noted, $\chi^{0}=1$ and $\chi^{k-1}$ is the signum character.

Lemma A.2. Let $1 \leq j \leq k-1$ and let $p \in \mathscr{P}$. Then

$$
\chi^{j}\left(C^{p}\right)=-\chi^{j-1}\left(C^{p}\right)+\left(\begin{array}{l}
k \\
j
\end{array}\right) \sum(-1)^{\sigma(\alpha)} \frac{\operatorname{Card}\left(C^{\alpha}\right) \operatorname{Card}\left(C^{\beta}\right)}{\operatorname{Card}\left(C^{p}\right)} .
$$

The summation in (5) is over all $\alpha, \beta \in \mathscr{P}$ with $w(\alpha)=j$ and $\alpha+\beta=p$. Proof. By formula (10) of $\S 4.3$ one has

$$
\chi_{j, k}=\frac{1}{k !} \sum_{p \in \mathscr{P}_{k}} \operatorname{Card}\left(C^{p}\right) \chi^{j}\left(C^{p}\right) s^{p} .
$$

Multiplying (1) and (2), we obtain

$$
q_{k-j} \boldsymbol{\Theta}_{j}=\frac{1}{j !(k-j) !} \sum \operatorname{Card}\left(C^{\alpha}\right) \operatorname{Card}\left(C^{\beta}\right)(-1)^{\sigma(\alpha)} s^{\alpha+\beta},
$$

where the sum is over $\alpha, \beta \in \mathscr{P}_{k}$ with $w(\alpha)=j$ and $w(\beta)=k-j$. Now we use (6) and (7) to calculate the coefficient of $s^{p}$ on each side of (3). This yields (5).

For each partition $p \in \mathscr{P}_{k}$, we define the generating function

$$
\phi_{p}(t)=\sum_{j=0}^{k-1}(-t)^{j} \chi^{j}\left(C^{p}\right) .
$$

This function can be expressed in terms of cyclotomic polynomials as follows: 
Lemma A.3. For $p \in \mathscr{P}_{k}$, one has

$$
(1-t) \phi_{p}(t)=\prod_{i}\left(1-t^{i}\right)^{p_{i}}
$$

Proof. Let $\alpha, \beta \in \mathscr{P}$ with $\alpha+\beta=p$. By formula (16) of $\S 4.3$, one calculates that

$$
\left(\begin{array}{l}
k \\
j
\end{array}\right) \frac{\operatorname{Card}\left(C^{\alpha}\right) \operatorname{Card}\left(C^{\beta}\right)}{\operatorname{Card}\left(C^{p}\right)}=\prod_{i}\left(\begin{array}{l}
p_{i} \\
\alpha_{i}
\end{array}\right) \text {. }
$$

Substituting this formula in (5), we obtain the expansion

$$
\begin{aligned}
\phi_{p}(t)=1 & +\sum_{j=0}^{k-2}(-1)^{j} t^{j+1} \chi^{j}\left(C^{p}\right) \\
& +\sum_{j=1}^{k-1}(-t)^{j} \sum_{\alpha \in \mathscr{P}_{j}}(-1)^{\sigma(\alpha)} \prod_{i}\left(\begin{array}{c}
p_{i} \\
\alpha_{i}
\end{array}\right) .
\end{aligned}
$$

For $\alpha \in \mathscr{P}$ one has

$$
(-1)^{w(\alpha)+\sigma(\alpha)}=(-1)^{|\alpha|} .
$$

Adding and subtracting the term $t^{k}(-1)^{k+\sigma(p)}$ from the right side of (10) and recalling that $\chi^{k-1}\left(C^{p}\right)=(-1)^{\sigma(p)}$, we thus can write (10) as

$$
\phi_{p}(t)=1+t \phi_{p}(t)+\sum_{j=1}^{k} \sum_{\alpha \in \mathscr{P}_{j}} \prod_{i}\left\{\left(\begin{array}{c}
p_{i} \\
\alpha_{i}
\end{array}\right)(-1)^{\alpha_{i}} t^{i \alpha_{i}}\right\} \text {. }
$$

In (11) we follow the usual convention with binomial coefficients:

$$
\left(\begin{array}{l}
a \\
b
\end{array}\right)=0 \text { if } b>a \text {. }
$$

Because of this, we may simply write (11) as

$$
(1-t) \phi_{p}(t)=\sum_{\alpha \in \mathscr{P}} \prod_{i}\left\{\left(\begin{array}{c}
p_{i} \\
\alpha_{i}
\end{array}\right)(-1)^{\alpha_{i}} t^{i \alpha_{i}}\right\},
$$

where the term with $\alpha=0$ contributes 1 to the right side of (12). But now we recognize the right side of (12) as the expansion of the right side of (9).

Proposition A.4. For $1 \leq j \leq k-1$ and $p \in \mathscr{P}_{k}$ one has

$$
(-1)^{j} \chi^{j}\left(C^{p}\right)=1+\sum_{m=1}^{j} \sum_{\alpha \in \mathscr{P}_{m}}(-1)^{|\alpha|} \prod_{i}\left(\begin{array}{c}
p_{i} \\
\alpha_{i}
\end{array}\right) \text {. }
$$

Proof. Multiply equation (12) by the geometric series expansion of $(1-t)^{-1}$. The coefficient of $t^{j}$ is then given by (13).

A.2. Proof of Lemma 4.2. We start with the well-known property

$$
\operatorname{dim}\left(\bigwedge^{3} \mathfrak{g}^{*}\right)^{G}=1
$$


of the cohomology of a simple Lie group, where the space (1) is spanned by the invariant 3-form $B([X, Y], Z)$, with $B$ the Killing form of $\mathfrak{g}$.

We shall show that (1) implies that $\operatorname{dim} \operatorname{Hom}_{G}\left(\mathfrak{g}, \bigwedge^{2} \mathfrak{g}\right)=1$. For this we introduce the following maps: Let

$$
\alpha: \bigwedge^{3} \mathfrak{g} \rightarrow \mathfrak{g} \otimes \bigwedge^{2} \mathfrak{g}
$$

be the inclusion map. Let

$$
\beta: \mathfrak{g} \otimes \bigwedge^{2} \mathfrak{g} \rightarrow S^{2}(\mathfrak{g}) \otimes \mathfrak{g}
$$

be the symmetrization map on the first and second indices. If $T \in \mathfrak{g} \otimes \bigwedge^{2} \mathfrak{g}$ has components $\left\{a_{i j k}\right\}$ relative to a basis $\left\{X_{i} \otimes X_{j} \otimes X_{k}\right\}$ for $\otimes^{3} \mathfrak{g}$, then $\beta(T)$ has components $\left\{\left(a_{i j k}+a_{j i k}\right) / 2\right\}$. In particular, if $T \in \operatorname{Image}(\alpha)$, then $a_{i j k}$ is skew in all indices, and hence $\beta(T)=0$. Conversely, if $\beta(T)=0$, then $a_{i j k}$ is skew in the first and second indices. Since it is already skew in the second and third indices, it must be completely antisymmetric, since the group $\Sigma_{3}$ is generated by the transpositions (12) and (23). Hence $T$ is in the subspace $\bigwedge^{3} \mathfrak{g}$. Thus we have

$$
\operatorname{Image}(\alpha)=\operatorname{Kernel}(\beta)
$$

Finally, let

$$
\gamma: S^{2}(\mathfrak{g}) \otimes \mathfrak{g} \rightarrow S^{3}(\mathfrak{g})
$$

be symmetrization relative to the second and third indices. By the same argument that gave (2), we see that

$$
\operatorname{Image}(\beta)=\operatorname{Kernel}(\gamma) .
$$

Obviously $\gamma$ is surjective, and the maps $\alpha, \beta, \gamma$ are $G$ homomorphisms. Restricting these maps to the corresponding subspaces of $G$-invariant tensors, we obtain from (2) and (3) the exact sequence of maps

$$
0 \rightarrow\left(\bigwedge^{3} \mathfrak{g}\right)^{G} \stackrel{\alpha}{\rightarrow}\left(\mathfrak{g} \otimes \bigwedge^{2} \mathfrak{g}\right)^{G} \stackrel{\beta}{\rightarrow}\left(S^{2}(\mathfrak{g}) \otimes \mathfrak{g}\right)^{G} \stackrel{\vartheta}{\rightarrow} S^{3}(\mathfrak{g})^{G} \rightarrow 0 .
$$

We claim that the map $\gamma$ in sequence (4) is an isomorphism. Indeed, given $u \in S^{3}(\mathfrak{g})^{G}$, we know by Corollary 1.2 that $T_{u} \in \operatorname{Hom}_{G}\left(\mathfrak{g}, S^{2}(\mathfrak{g})\right)$. If we identify $\left(S^{2}(\mathfrak{g}) \otimes \mathfrak{g}\right)^{G}$ with $\operatorname{Hom}_{G}\left(\mathfrak{g}, S^{2}(\mathfrak{g})\right)$ using the Killing form, then we find that $\gamma\left(T_{u}\right)=3 u$. Thus the map $u \rightarrow \frac{1}{3} T_{u}$ is the inverse of $\gamma$ on the space $S^{3}(\mathfrak{g})^{G}$. Now from the exactness of sequence (4) we thus conclude that $\beta=0$, and hence $\alpha$ is an isomorphism on the $G$-invariants. By (1) we conclude that

$$
\operatorname{dim}\left(\mathfrak{g} \otimes \bigwedge^{2} \mathfrak{g}\right)^{G}=1
$$

Since $\operatorname{Hom}\left(\mathfrak{g}, \bigwedge^{2} \mathfrak{g}\right)$ is isomorphic to $\mathfrak{g} \otimes \Lambda^{2} \mathfrak{g}$ as a $G$-module, this proves Lemma 4.2. 
Note added in proof. The paper of T. Hayashi mentioned in the Introduction has appeared in Invent. Math. 94 (1988), 13-52. Other recent papers in the physics literature on higher-order Sugawara operators include the following.

F. A. Bais, P. Bouwknegt, M. Surridge and K. Schoutens, Extensions of the Virasoro algebra constructed from Kac-Moody algebras using higher order Casimir invariants, Nuclear Phys. B304 (1988), 348-370. , Coset construction for extended Virasoro algebras, Nuclear Phys. B304 (1988), 371-391.

\section{REFERENCES}

[C-I] V. Chari and S. Ilangovan, On the Harish-Chandra homomorphism for infinite-dimensional Lie algebras, J. Algebra 90 (1984), 476-490.

[C-G-J] S. Coleman, D. Gross, and R. Jackiw, Fermion avatars of the Sugawara model, Phys. Rev. 180 (1969), 1359-1366.

[Fre] I. B. Frenkel, Two constructions of affine Lie algebra representations and boson-fermion correspondence in quantum field theory, J. Funct. Anal. 44 (1981), 259-327.

[G-O] P. Goddard and D. Olive, Kac-Moody and Virasoro algebras in relation to quantum physics, Internat. J. Mod. Phys. A 1 (1986), 303-414.

[Goo] R. Goodman, Nilpotent Lie groups: structure and applications to analysis, Lecture Notes in Math., vol. 562, Springer-Verlag, Berlin, 1976.

[G-W1] R. Goodman and N. R. Wallach, Whittaker vectors and conical vectors, J. Funct. Anal. 39 (1980), 199-279.

[G-W2] _ Classical and quantum-mechanical systems of Toda lattice type. I, Comm. Math. Phys. 83 (1982), 355-386.

[G-W3] _ Structure and unitary cocycle representations of loop groups and the group of diffeomorphisms of the circle, J. Reine Angew. Math. 347 (1984), 69-133; erratum, 352, 220.

[G-W4] _ Classical and quantum-mechanical systems of Toda lattice type. III. Comm. Math. Phys. 105 (1986), 473-509.

[Har] Harish-Chandra, Differential operators on a semi-simple Lie algebra, Amer. J. Math. 79 (1957), 87-120.

[Kac] V. Kac, Laplace operators of infinite-dimensional Lie algebras and theta functions, Proc. Nat. Acad. Sci. U.S.A. 81 (1984), 645-647.

[Kos] B. Kostant, Whittaker vectors and representation theory, Invent. Math. 48 (1978), 101-184.

[K-K] V. Kac and D. A. Kazhdan, Structure of representations with highest weight of infinitedimensional Lie algebra, Adv. in Math. 34 (1979), 97-108.

[L-W] J. Lepowsky and R. L. Wilson, The structure of standard modules, I: universal algebras and the Rogers-Ramanujan identities, Invent. Math. 77 (1984), 199-290.

[Mur] F. D. Murnaghan, The theory of group representations, Dover, New York, 1963.

[P-S] A. Pressley and G. Segal, Loop groups, Oxford Univ. Press, New York, 1986.

[Sug] H. Sugawara, A field theory of currents, Phys. Rev. 170 (1968), 1659-1662.

[Wal] N. R. Wallach, A class of non-standard modules for affine Lie algebras, Math. Z. 196 (1987), 303-313.

[Wey] H. Weyl, The classical groups, Princeton Univ. Press, Princeton, N.J., 1946.

Department of Mathematics, Rutgers University, New Brunswick, New Jersey 08903 